\title{
Ruthenium-Catalyzed Asymmetric $N$-Acyl Nitrene Transfer Reaction: Imidation of Sulfide
}

\author{
Masaki Yoshitake, ${ }^{[b]}$ Hiroki Hayashi, ${ }^{[a]}$ and Tatsuya Uchida, ${ }^{*[a, c]}$ \\ [a] Faculty of Arts and Science, Kyushu University, 744 Motooka, Nishi-ku, Fukuoka, 819- \\ 0395, Japan \\ [b] Department of Chemistry, Faculty of Science, Graduate School, Kyushu University, 744 \\ Motooka, Nishi-ku, Fukuoka, 819-0395, Japan \\ [c] International Institute for Carbon-Neutral Energy Research (WPI-I2CNER), Kyushu \\ University, 744 Motooka, Nishi-ku, Fukuoka, 819-0395, Japan.
}

\begin{abstract}
The asymmetric nitrene transfer reaction is useful and strong tool for the construction of nitrogen functional groups such as $N$-sulfonyl amide, carbamic ester in a highly enantioselective manner. On the other hand, there is a large limitation in this filed: the transfer of $\mathrm{N}$-acyl amide via the corresponding nitrene intermediates is still difficult because $N$-acyl nitrenes underwent undesired nitrene dimerization or Curtius rearrangement. Herein, we achieved highly enantioselective imidation of sulfides via catalytic $N$-acyl nitrene transfer with (OC)ruthenium-salen complex $\mathbf{2} \mathbf{b}$ as the catalyst and 3-sbstituted-1,4,2-dioxazol-5-ones $\mathbf{1}$ as the nitrene source. Complex $\mathbf{2 b}$ can decompose dioxazolones $\mathbf{1}$ to the desired $\mathrm{N}$-acyl nitrene intermediates without any activation such as heating and UV-irradiation, and transfer generating nitrene intermediates to sulfur atom of sulfides with good to excellent enantioselectivities (up to $98 \%$ ee) without contamination of diazenes and isocyanates.
\end{abstract}




\section{Content}

1. General information........................................................S3

2. Optimization of reaction conditions......................................S4

3. General procedure..........................................................

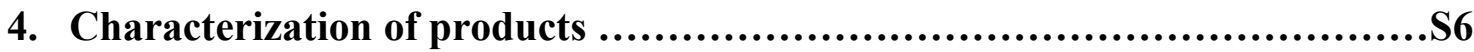

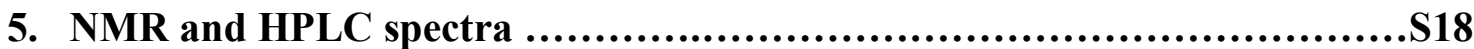

6. The Chromatograms for the determination of enantioselectivity..............S53

7. References..................................................................S62 


\section{General information}

${ }^{1} \mathrm{H}$ NMR and ${ }^{13} \mathrm{C}$ NMR spectra were recorded on JEOL JNM-AL-400 spectrometer at 400 and $100 \mathrm{MHz}$, respectively. Chemical shifts were expressed in parts per million (ppm) with respect to tetramethylsilane (TMS) as the internal standard $(0 \mathrm{ppm})$ for ${ }^{1} \mathrm{H} \mathrm{NMR}$, and $\mathrm{CDCl}_{3}$, $\mathrm{CD}_{3} \mathrm{OD}$, and DMSO- $\mathrm{d}_{6}$ as internal standard $\left(77.0,49.0\right.$, and $39.7 \mathrm{ppm}$, respectively) for ${ }^{13} \mathrm{C}$ NMR. Coupling constants were reported as Hertz (Hz), signal shapes and splitting patterns were indicated as follows: s, singlet; br, broad; d, doublet; t. triplet; q, quartet; dd, doublet doublet; $\mathrm{m}$, multiplet. Mass spectra were recorded on a BRUKER DALTONICS MICRO TOF-KSI focus (HR-ESI); peaks are given in $\mathrm{m} / \mathrm{z}$ (\% of basis peak). Enantiomeric excesses were determined by HPLC analysis using SHIMADZU HPLC Prominence equipped with a column packed with an appropriate optically active material, as described below. Infrared spectra were obtained with SHIMADZU IRAffinity-1 FTIR spectrophotometer, and only diagnostic signals are listed below. Optical rotations were recorded on an ANTON PAAR MCL-300. Thin layer chromatography was performed on Merck silica gel 60 F254 coated glass plates and visualized by fluorescence quenching under UV light or staining with the standard solution of $12 \mathrm{MoO}_{3} \cdot \mathrm{H}_{3} \mathrm{PO}_{4}$. Column chromatography was conducted on Cica (Kanto Chemical Co., INC.) silica gel 60N (spherical, neutral, 63-210 $\mu \mathrm{m}$ ). All commercially available regents were purchased from TCI (Tokyo Chemical Industry Co., LTD.), SigumaAldrich, Cica, Fujifilm Wako Pure Chemical Corporation in the highest purity grade and distilled before use. 3-Substituted-1,2,4-dioxazolones 1a-t and (OC)Ruthenium-salen complexes 2a-k were prepared according to the reported procedures. ${ }^{1,2}$ 


\section{Optimization of reaction conditions.}

Table S1. Optimization of enantioselective $N$-acyl imidation of methyl phenyl sulfide 3a with ruthenium complexes 2 as catalysts and 3-phenyl-1,4,2-dioxazol-5-one 1a as the nitrene source. ${ }^{a}$

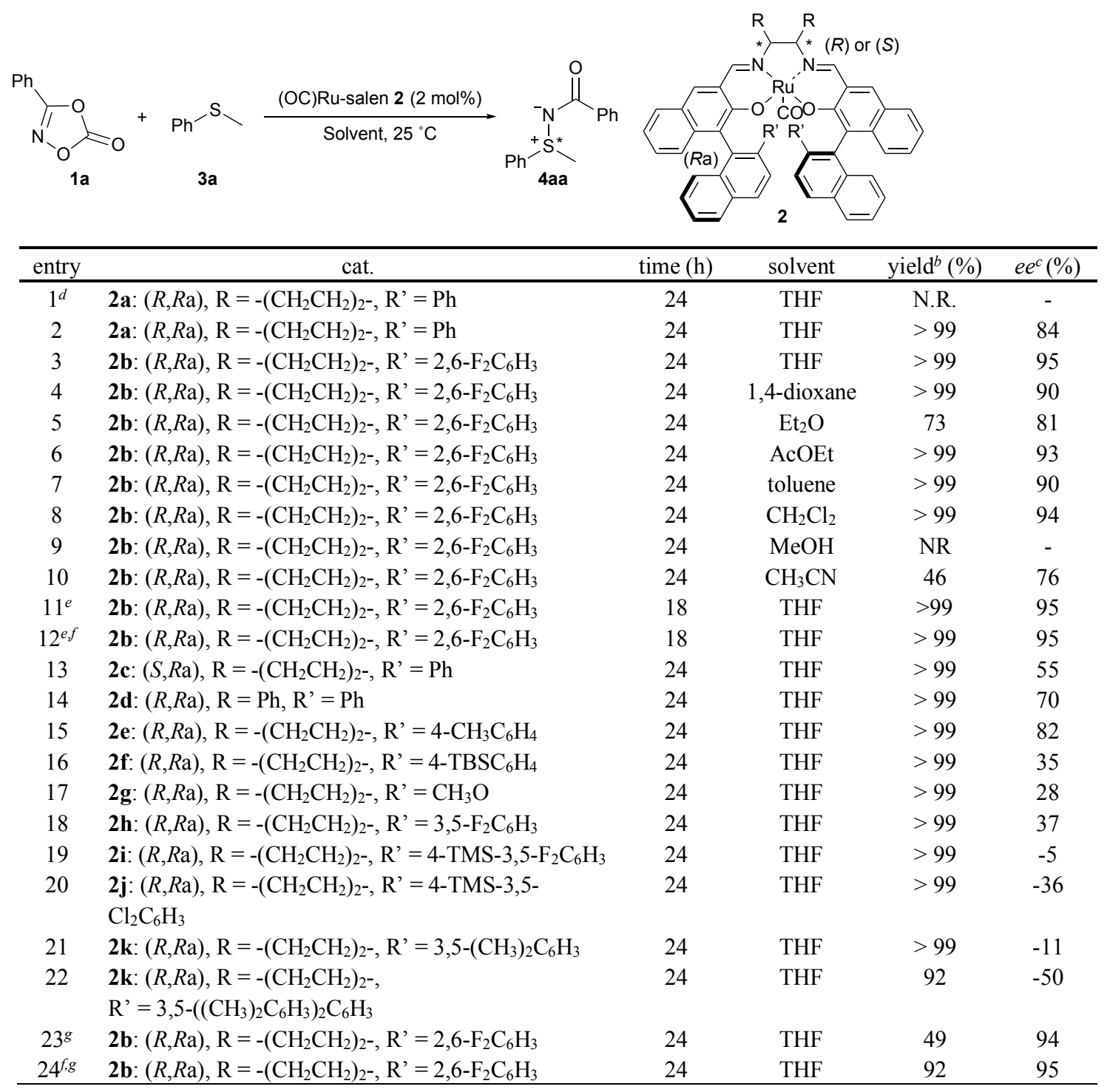

${ }^{a}$ Reactions were carried out using 1a $(0.13 \mathrm{mmol})$ and $\mathbf{3 a}(0.1 \mathrm{mmol})$ with ruthenium complex $\mathbf{2}(2 \mathrm{~mol} \%)$ at $25{ }^{\circ} \mathrm{C}$, unless otherwise specified. ${ }^{b}$ Isolated yield. ${ }^{c}$ Determined by HPLC with chiral stationary phase. ${ }^{d} N$-benzoyl azide $(0.13 \mathrm{mmol})$ was used as the nitrene source instead of 1a. ${ }^{e}$ Carried out with $1.0 \mathrm{~mol} \%$ loading of $\mathbf{2 b} .{ }^{f}$ Added MS $4 \AA$ ( $20 \mathrm{mg}$ ). ${ }^{g}$ Carried out with $0.5 \mathrm{~mol} \%$ loading of $\mathbf{2 b}$. 


\section{General procedure}

\subsection{Typical reaction conditions of asymmetric $N$-acyl imidation of sulfide 3 .}

Sulfide 3 (0.1 mmol), (OC)ruthenium-salen $2 \mathbf{b}(1.0 \mathrm{mg}, 1.0 \mu \mathrm{mol}, 1.0 \mathrm{~mol} \%)$, molecular sieves $4 \AA(20 \mathrm{mg})$, and THF $(0.5 \mathrm{~mL})$ were placed in $5.0 \mathrm{~mL}$ of Schlenk tube and the mixture was stirred for over $30 \mathrm{~min}$ at $25{ }^{\circ} \mathrm{C}$ under nitrogen atmosphere. At that temperature, 3substituted-1,4,2-dioxazolone $1(0.13 \mathrm{mmol})$ was added to the mixture. After stirred for $18 \mathrm{~h}$, the reaction mixture was concentrated on a rotatory evaporator. The residue was purified on a silica gel chromatography with hexane/ethyl acetate to afford the desired product 4 . The enantiomeric excess of the product $\mathbf{4}$ was determined by HPLC analysis on an appropriate chiral stationary phase column.

\subsection{Imidation of methyl phenyl sulfide 3a using 3-phenyl-1,4,2-dioxazolone 1 a.}

Methyl phenyl sulfide 3a $(117 \mu \mathrm{L}, 1.0 \mathrm{mmol})$, (OC)ruthenium-salen $\mathbf{2 b}(2.0 \mathrm{mg}, 2.0 \mu \mathrm{mol}$, $0.2 \mathrm{~mol} \%)$, molecular sieves $4 \AA(20 \mathrm{mg})$, and THF $(0.5 \mathrm{~mL})$ were placed in $5.0 \mathrm{~mL}$ of Schlenk tube and the mixture was stirred for over $30 \mathrm{~min}$ at $25{ }^{\circ} \mathrm{C}$ under nitrogen atmosphere. At that temperature, 3-phenyl-1,4,2-dioxazol-5-one $1 \mathrm{a}(212 \mathrm{mg}, 1.3 \mathrm{mmol})$ was added to the mixture. After stirred for $24 \mathrm{~h}$, the reaction mixture was concentrated on a rotatory evaporator. Resulting residue was purified by silica gel chromatography ( $n$-hexane/AcOEt $=1 / 1$ to $3 / 7$ ) to affored the desired $N$-benzoyl methyl phenyl sulfimide 4 aa $(214.8 \mathrm{mg}, 0.88 \mathrm{mmol}, 88 \%$ yield).

\subsection{Typical preparation of racemic $\mathbf{N}$-acyl sulfimides 4 .}

Sulfide 3 (0.1 mmol), (OC)ruthenium-salophen (1.34 mg, $2.0 \mu \mathrm{mol}, 2 \mathrm{~mol} \%)$, molecular sieves $4 \AA(20 \mathrm{mg})$, and THF $(0.5 \mathrm{~mL})$ were placed in $5.0 \mathrm{~mL}$ of Schlenk tube and the mixture was stirred for over $30 \mathrm{~min}$ at $25{ }^{\circ} \mathrm{C}$ under nitrogen atmosphere. At that temperature, 3substituted-1,4,2-dioxazol-5-one $1(0.13 \mathrm{mmol})$ was added to the mixture. After stirred for $18 \mathrm{~h}$, the reaction mixture was concentrated on a rotatory evaporator. The residue was purified on a silica gel chromatography with hexane/ethyl acetate (1/1 to $3 / 7)$ to afford the desired product 4 .

\subsection{Preparation of $\mathbf{N}$-acyl sulfoximine $5 .^{3}$}

To the $\mathrm{CH}_{3} \mathrm{CN} / \mathrm{H}_{2} \mathrm{O}(2 / 1,3 \mathrm{~mL})$ solution of sulfimide $4(0.1 \mathrm{mmol})$, sodium periodide $(107$ $\mathrm{mg}, 0.4 \mathrm{mmol})$ and ruthenium(III) chloride 3-hydrate $(1.31 \mathrm{mg}, 5 \mu \mathrm{mol}, 5 \mathrm{~mol} \%)$ were added at room temperature. After stirred for $1 \mathrm{~h}$, the mixture was extracted with dichloromethane (3 x $5 \mathrm{~mL})$. The combined organic layers were washed with brine $(5 \mathrm{~mL})$. The organic phase was dried over anhydrous $\mathrm{Na}_{2} \mathrm{SO}_{4}$, filtered, concentrated with evaporation. The residue was subjected to chromatography on a silica gel with hexane/ethyl acetate (3/7) to give the desired product 5 . 


\section{Characterization Data of the products.}

\section{1. (-)- $N$-Benzoyl methyl phenyl sulfimide (4aa)}

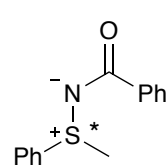

Following typical experiment 3.1, 3-phenyl-1,4,2-dioxazol-5-one 1a (212 mg, $1.3 \mathrm{mmol})$ and methyl phenyl sulfide 3a $(117 \mu \mathrm{L}, 1.0 \mathrm{mmol})$ with (OC)ruthenium-salen $\mathbf{2 b}(2.1 \mathrm{mg}, 2.0 \mu \mathrm{mol}, 0.2 \mathrm{~mol} \%)$ as the catalyst in THF $(1.0 \mathrm{~mL})$, after chromatography $(n$-hexane/AcOEt $=1 / 1$ to $3 / 7)$ the corresponding $N$-benzoyl sulfimide 4 aa was obtained as white solid $(215 \mathrm{mg}$, 88\%).; Colorless crystals were obtained from $\mathrm{CHCl}_{3} / \mathrm{Hexane}$.; m.p. 104.5-104.6 ${ }^{\circ} \mathrm{C}$.; The enantiomeric excess of the sulfimide 4aa was determined to be $97 \%$ ee by HPLC analysis using a chiral stationary phase [DAICEL CHIRALPAK IF-3 (Hexane $/ i$-PrOH $=6 / 4,0.5$ $\mathrm{mL} / \mathrm{min}), t_{\mathrm{r}}($ Major $)=19.2 \mathrm{~min}, t_{\mathrm{r}}($ Minor $\left.)=16.5 \mathrm{~min}\right]$; white solid; $[\alpha]_{\mathrm{D}}{ }^{25}=-151.7(c=1.20$, $\left.\mathrm{CHCl}_{3}\right) ;{ }^{1} \mathrm{H} \mathrm{NMR}\left(400 \mathrm{MHz}, \mathrm{CDCl}_{3}\right) \delta 8.17(\mathrm{~d}, J=7.3 \mathrm{~Hz}, 2 \mathrm{H}), 7.84(\mathrm{dd}, J=2.4,7.3 \mathrm{~Hz}$, $2 \mathrm{H}), 7.56-7.52(\mathrm{~m}, 3 \mathrm{H}), 7.46-7.37(\mathrm{~m}, 3 \mathrm{H}), 2.92$ (s, 3H) ppm; ${ }^{13} \mathrm{C} \mathrm{NMR}\left(100 \mathrm{MHz}, \mathrm{CDCl}_{3}\right)$ : $\delta 176.8,136.3,135.9,132.1,130.8,129.9,128.7,127.8,126.7$ ppm; FT-IR (KBr): 3557, 3013, 2922, 1549, 1481, 1439, 1412, 1329, 1069, 1020, 972, 868, 806, 750, $716 \mathrm{~cm}^{-1}$; HRESI-MS $(\mathrm{m} / \mathrm{z})\left[\mathrm{M}+\mathrm{Na}^{+}\right]$, calcd for $\left[\mathrm{C}_{14} \mathrm{H}_{13} \mathrm{NNaOS}\right]^{+} 266.0610$, found: $\mathrm{m} / \mathrm{z}=266.0610$.

\section{2. (-)- $N$-(4-Methylbenzoyl) methyl phenyl sulfimide (4ba)}<smiles>Cc1ccc(C(=O)N(C)[SH](C)c2ccccc2)cc1</smiles>

Following typical experiment 3.1, 3-(4-methylphenyl)-1,4,2-dioxazol-5one $1 \mathbf{b}(23.0 \mathrm{mg}, 0.13 \mathrm{mmol})$ and methyl phenyl sulfide $3 \mathbf{a}(11.8 \mu \mathrm{L}, 0.1$ mmol), after chromatography ( $n$-hexane/AcOEt $=1 / 1$ to $3 / 7$ ) the corresponding $N$-benzoyl sulfimide $\mathbf{4 b a}$ was obtained as white solid (25.7 $\mathrm{mg},>99 \%$ ); The enantiomeric excess of the sulfimide $4 \mathbf{b a}$ was determined to be $96 \%$ ee by HPLC analysis using a chiral stationary phase [DAICEL CHIRALPAK IF-3 (Hexane $/ i-\mathrm{PrOH}=6 / 4,0.5 \mathrm{~mL} / \mathrm{min}), t_{\mathrm{r}}$ (Major) $=21.4 \mathrm{~min}, t_{\mathrm{r}}$ (Minor) $=$ $18.7 \mathrm{~min}]$; white solid; $[\alpha]_{\mathrm{D}}{ }^{25}=-132.5\left(c=1.19, \mathrm{CHCl}_{3}\right),{ }^{1} \mathrm{H}$ NMR $\left(400 \mathrm{MHz}, \mathrm{CDCl}_{3}\right) \delta 8.07$ $(\mathrm{d}, J=8.3 \mathrm{~Hz}, 2 \mathrm{H}), 7.83(\mathrm{~d}, J=7.3,2.4 \mathrm{~Hz}, 2 \mathrm{H}), 7.54-7.50(\mathrm{~m}, 3 \mathrm{H}), 7.18(\mathrm{~d}, J=8.3 \mathrm{~Hz}, 2 \mathrm{H})$, $2.90(\mathrm{~s}, 3 \mathrm{H}), 2.38(\mathrm{~s}, 3 \mathrm{H}) \mathrm{ppm} ;{ }^{13} \mathrm{C}$ NMR $\left(100 \mathrm{MHz}, \mathrm{CDCl}_{3}\right) \delta 176.8,140.9,136.1,133.5,132.0$, 129.9, 128.7, 128.5, 126.6, 35.1, 21.5 ppm; FT-IR (KBr): 3055, 3015, 2922, 1585, 1543, 1412, $1165,1126,1096,972,841,748,685 \mathrm{~cm}^{-1}$; HRESI-MS $(\mathrm{m} / \mathrm{z})\left[\mathrm{M}+\mathrm{Na}^{+}\right]$, calcd for $\left[\mathrm{C}_{15} \mathrm{H}_{15} \mathrm{NNaOS}\right]^{+} 280.0767$, found: $\mathrm{m} / \mathrm{z}=280.0771$.

\section{3. (-)- $N$-(4-Methoxylbenzoyl) methyl phenyl sulfimide (4ca)}<smiles>COc1ccc(C(=O)N(C)[SH](C)(=S)c2ccccc2)cc1</smiles>

Following typical experiment 3.1, 3-(4-methoxyphenyl)-1,4,2dioxazol-5-one 1c (25.1 $\mathrm{mg}, 0.13 \mathrm{mmol})$ and methyl phenyl sulfide $\mathbf{3 a}$ $(11.8 \mu \mathrm{L}, 0.1 \mathrm{mmol})$, after chromatography ( $n$-hexane $/ \mathrm{AcOEt}=1 / 1$ to $3 / 7$ ) the corresponding $N$-benzoyl sulfimide 4 ca was obtained as white solid (26.9 mg, 99\%).; The enantiomeric excess of the sulfimide 4ca was determined to be $98 \%$ ee by HPLC analysis using a chiral stationary phase [DAICEL CHIRALPAK IF-3 (Hexane $/ i-\mathrm{PrOH}=6 / 4,0.5 \mathrm{~mL} / \mathrm{min}), t_{\mathrm{r}}($ Major $)=28.4 \mathrm{~min}, t_{\mathrm{r}}($ Minor $)=$ $24.6 \mathrm{~min}]$; white solid; $[\alpha]_{\mathrm{D}}{ }^{25}=-109.7\left(c=1.22, \mathrm{CHCl}_{3}\right) ;{ }^{1} \mathrm{H}$ NMR $\left(400 \mathrm{MHz}, \mathrm{CDCl}_{3}\right) \delta 8.13$ $(\mathrm{d}, J=8.8 \mathrm{~Hz}, 2 \mathrm{H}), 7.83(\mathrm{~d}, J=7.3,2.0 \mathrm{~Hz}, 2 \mathrm{H}), 7.57-7.51(\mathrm{~m}, 3 \mathrm{H}), 6.89(\mathrm{~d}, J=8.8 \mathrm{~Hz}, 2 \mathrm{H})$, $3.84(\mathrm{~s}, 3 \mathrm{H}), 2.91(\mathrm{~s}, 3 \mathrm{H}) \mathrm{ppm} ;{ }^{13} \mathrm{C} \mathrm{NMR}\left(100 \mathrm{MHz}, \mathrm{CDCl}_{3}\right) \delta 176.5,161.7,136.2,130.5$, 129.07, 126.6, 113.0, 55.3, 35.2 ppm; FT-IR (KBr): 3061, 3007, 2924, 2841, 1668, 1597, $1545,1329,1248,1171,1026,968,851,816,691,604 \mathrm{~cm}^{-1}$; HRESI-MS (m/z) $\left[\mathrm{M}+\mathrm{Na}^{+}\right]$, calcd for $\left[\mathrm{C}_{15} \mathrm{H}_{15} \mathrm{NNaO}_{2} \mathrm{~S}\right]^{+} 296.0716$, found: $\mathrm{m} / \mathrm{z}=296.0716$. 


\section{4. (-)- $N$-(4-Bromobenzoyl) methyl phenyl sulfimide (4da)}<smiles></smiles>

Following typical experiment 3.1, 3-(4-bromophenyl)-1,4,2-dioxazol-5one 1d $(31.3 \mathrm{mg}, 0.13 \mathrm{mmol})$ and methyl phenyl sulfide 3a $(11.8 \mu \mathrm{L}, 0.1$ mmol), after chromatography ( $n$-hexane/AcOEt $=1 / 1$ to $3 / 7$ ) the corresponding $N$-benzoyl sulfimide 4da was obtained as white solid (31.9 $\mathrm{mg}, 99 \%$ ).; The enantiomeric excess of the sulfimide 4da was determined to be $95 \%$ ee by HPLC analysis using a chiral stationary phase [DAICEL CHIRALPAK IF$3(\mathrm{Hexane} / i-\mathrm{PrOH}=6 / 4,0.5 \mathrm{~mL} / \mathrm{min}), t_{\mathrm{r}}($ Major $)=19.6 \mathrm{~min}, t_{\mathrm{r}}($ Minor $\left.)=17.7 \mathrm{~min}\right]$; white solid; $[\alpha]_{\mathrm{D}}{ }^{25}=-81.5\left(c=1.25, \mathrm{CHCl}_{3}\right) ;{ }^{1} \mathrm{H}$ NMR $\left(400 \mathrm{MHz}, \mathrm{CDCl}_{3}\right) \delta 8.04(\mathrm{~d}, J=8.3 \mathrm{~Hz}$, 2H), $7.83(\mathrm{dd}, J=7.8,2.0 \mathrm{~Hz}, 2 \mathrm{H}), 7.50-7.68(\mathrm{~m}, 5 \mathrm{H}), 2.92(\mathrm{~s}, 3 \mathrm{H}) \mathrm{ppm} ;{ }^{13} \mathrm{C}$ NMR $(100 \mathrm{MHz}$, $\left.\mathrm{CDCl}_{3}\right) \delta 175.7,135.6,135.2,132.3,131.0,130.4,130.0,126.7,125.5,35.1 \mathrm{ppm} ;$ FT-IR (KBr): 3539, 3445, 3065, 1626, 1587, 1558, 1506, 1450, 1395, 1248, 1206, 1150, 1099, 993 , $881,853,800,748 \mathrm{~cm}^{-1}$; HRESI-MS $(\mathrm{m} / \mathrm{z})\left[\mathrm{M}+\mathrm{Na}^{+}\right]$, calcd for $\left[\mathrm{C}_{14} \mathrm{H}_{12} \mathrm{BrNNaOS}\right]^{+} 343.9715$, found: $\mathrm{m} / \mathrm{z}=343.9713$.

\section{5. (-)- $N$-(4-Chlorobenzoyl) methyl phenyl sulfimide (4ea)}<smiles>C[SH](=[W])(/N=C(/N)C(=O)c1ccc(Cl)cc1)c1ccccc1</smiles>

Following typical experiment 3.1, 3-(4-chlorophenyl)-1,4,2-dioxazol-5one 1e $(25.6 \mathrm{mg}, 0.13 \mathrm{mmol})$ and methyl phenyl sulfide $3 \mathbf{a}(11.8 \mu \mathrm{L}, 0.1$ mmol), after chromatography ( $n$-hexane/AcOEt $=1 / 1$ to $3 / 7)$ the corresponding $N$-benzoyl sulfimide 4ea was obtained as white solid (25.9 $\mathrm{mg}, 93 \%$ ).; The enantiomeric excess of the sulfimide 4 ea was determined to be $94 \%$ ee by HPLC analysis using a chiral stationary phase [DAICEL CHIRALPAK IF-3 (Hexane $/ i$-PrOH $=6 / 4,0.5 \mathrm{~mL} / \mathrm{min}), t_{\mathrm{r}}($ Major $)=18.2 \mathrm{~min}, t_{\mathrm{r}}($ Minor $\left.)=16.5 \mathrm{~min}\right] . ;$ white solid; $[\alpha]_{\mathrm{D}}{ }^{25}=-94.5$ $\left(c=1.25, \mathrm{CHCl}_{3}\right) ;{ }^{1} \mathrm{H} \mathrm{NMR}\left(400 \mathrm{MHz}, \mathrm{CDCl}_{3}\right) \delta 8.11(\mathrm{~d}, J=8.8 \mathrm{~Hz}, 2 \mathrm{H}), 7.83(\mathrm{dd}, J=7.8$, $2.0 \mathrm{~Hz}, 2 \mathrm{H}), 7.58-7.53(\mathrm{~m}, 3 \mathrm{H}), 7.35(\mathrm{~d}, J=8.3 \mathrm{~Hz}, 2 \mathrm{H}), 2.93(\mathrm{~s}, 3 \mathrm{H}) \mathrm{ppm} ;{ }^{13} \mathrm{C}$ NMR $(100$ $\left.\mathrm{MHz}, \mathrm{CDCl}_{3}\right) \delta 175.7,136.9,135.7,134.8,132.3,130.2,130.0,128.0,126.7,35.2 \mathrm{ppm} ; \mathrm{FT}-$ IR (KBr): 3061, 3011, 2922, 1672, 1593, 1547, 1481, 1441, 1404, 1323, 1167, 1130, 1088, $1011,970,860,810,754,689,523 \mathrm{~cm}^{-1}$; HRESI-MS $(\mathrm{m} / \mathrm{z})\left[\mathrm{M}+\mathrm{Na}^{+}\right]$, calcd for $\left[\mathrm{C}_{14} \mathrm{H}_{12} \mathrm{ClNNaOS}\right]^{+} 300.0220$, found: $\mathrm{m} / \mathrm{z}=300.0221$.

\section{6. (-)- $N$-(4-Fluorobenzoyl) methyl phenyl sulfimide (4fa)}<smiles></smiles>

Following typical experiment 3.1, 3-(4-fluorophenyl)-1,4,2-dioxazol-5one 1 f $(23.5 \mathrm{mg}, 0.13 \mathrm{mmol})$ and methyl phenyl sulfide $\mathbf{3 a}(11.8 \mu \mathrm{L}, 0.1$ mmol), after chromatography ( $n$-hexane/AcOEt $=1 / 1$ to $3 / 7)$ the corresponding $N$-benzoyl sulfimide 4 fa was obtained as white solid (26.0 $\mathrm{mg},>99 \%$ ).; The enantiomeric excess of the sulfimide $4 \mathrm{fa}$ was determined to be $93 \%$ ee by HPLC analysis using a chiral stationary phase [DAICEL CHIRALPAK IF-3 (Hexane $/ i-\mathrm{PrOH}=6 / 4,0.5 \mathrm{~mL} / \mathrm{min}), t_{\mathrm{r}}($ Major $)=16.2 \mathrm{~min}, t_{\mathrm{r}}($ Minor $)=$ $14.8 \mathrm{~min}]$; white solid; $[\alpha]_{\mathrm{D}}{ }^{25}=-129.2\left(c=1.06, \mathrm{CHCl}_{3}\right) ;{ }^{1} \mathrm{H} \mathrm{NMR}\left(400 \mathrm{MHz}, \mathrm{CDCl}_{3}\right) \delta 8.17$ $(\mathrm{dd}, J=8.8,5.4 \mathrm{~Hz}, 2 \mathrm{H}), 7.83(\mathrm{dd}, J=7.6,2.2 \mathrm{~Hz}, 2 \mathrm{H}), 7.58-7.53(\mathrm{~m}, 3 \mathrm{H}), 7.04$ (psude-t, $J$ $=8.5 \mathrm{~Hz}, 2 \mathrm{H}), 2.92(\mathrm{~s}, 3 \mathrm{H}) \mathrm{ppm} ;{ }^{13} \mathrm{C} \mathrm{NMR}\left(100 \mathrm{MHz}, \mathrm{CDCl}_{3}\right) \delta 175.0,161.0(\mathrm{~d}, J=249.1$ $\mathrm{Hz}), 135.4,132.2,131.6(\mathrm{~d}, J=8.3 \mathrm{~Hz}), 131.3,130.0,126.8,125.4,123.5(\mathrm{~d}, J=4.1 \mathrm{~Hz})$, $116.4(\mathrm{~d}, J=23.2 \mathrm{~Hz}), 35.1 \mathrm{ppm}$; FT-IR (KBr): 3105, 3049, 2926, 2854, 1597, 1551, 1491, $1412,1321,1207,1138,1080,976,849,804,739,681,592 \mathrm{~cm}^{-1}$; HRESI-MS (m/z) [M + $\mathrm{Na}^{+}$, calcd for $\left[\mathrm{C}_{14} \mathrm{H}_{12} \mathrm{FNNaOS}\right]^{+} 284.0516$, found: $\mathrm{m} / \mathrm{z}=284.0517$. 


\section{7. (-)-N-(4-Trifluoromethylbenzoyl) methyl phenyl sulfimide (4ga)}<smiles>CN(C(=O)c1ccc(C(F)(F)F)cc1)S(C)(C)c1ccccc1</smiles>

Following typical experiment 3.1, 3-(4-trifluoromethylphenyl)-1,4,2dioxazol-5-one $1 \mathrm{~g}(30.0 \mathrm{mg}, 0.13 \mathrm{mmol})$ and methyl phenyl sulfide 3a $(11.8 \mu \mathrm{L}, 0.1 \mathrm{mmol})$, after chromatography $(n$-hexane/AcOEt $=1 / 1$ to $3 / 7$ ) the corresponding $N$-benzoyl sulfimide 4ga was obtained as white solid (15.9 mg, 51\%); The enantiomeric excess of the sulfimide 4ga was determined to be $91 \%$ ee by HPLC analysis using a chiral stationary phase [DAICEL CHIRALPAK IF-3 (Hexane $/ i-\mathrm{PrOH}=8 / 2,0.5 \mathrm{~mL} / \mathrm{min}), t_{\mathrm{r}}$ (Major) $=23.4 \mathrm{~min}, t_{\mathrm{r}}($ Minor $)=$ 21.9 min]; white solid; [ $\alpha]_{\mathrm{D}}{ }^{25}=-101.1\left(c=1.46, \mathrm{CHCl}_{3}\right) ;{ }^{1} \mathrm{H}$ NMR $\left(400 \mathrm{MHz}, \mathrm{CDCl}_{3}\right) \delta 8.26$ $(\mathrm{d}, J=8.3 \mathrm{~Hz}, 2 \mathrm{H}), 7.83(\mathrm{dd}, J=7.8,2.0 \mathrm{~Hz}, 2 \mathrm{H}), 7.63(\mathrm{~d}, J=8.3 \mathrm{~Hz}, 2 \mathrm{H}), 7.58-7.53(\mathrm{~m}$, $3 \mathrm{H}), 2.94(\mathrm{~s}, 3 \mathrm{H}) \mathrm{ppm} ;{ }^{13} \mathrm{C} \mathrm{NMR}\left(100 \mathrm{MHz}, \mathrm{CDCl}_{3}\right) \delta 175.2,139.6,135.4,132.4,132.3(\mathrm{q}$, $J=32.3 \mathrm{~Hz}), 130.0,129.0,126.7,124.8(\mathrm{q}, J=3.3 \mathrm{~Hz}), 124.1$ (q, $J=272.3 \mathrm{~Hz}), 35.1 \mathrm{ppm}$; FT-IR (KBr): 3539, 3445, 3065, 1626, 1587, 1558, 1506, 1450, 1395, 1248, 1206, 1150, 1099 , 993, 881, 853, 800, $748 \mathrm{~cm}^{-1}$; HRESI-MS $(\mathrm{m} / \mathrm{z})\left[\mathrm{M}+\mathrm{Na}^{+}\right]$, calcd for $\left[\mathrm{C}_{15} \mathrm{H}_{12} \mathrm{~F}_{3} \mathrm{NNaOS}\right]^{+}$ 334.0484, found: $\mathrm{m} / \mathrm{z}=334.0483$.

\section{8. (-)- $N$-(3-Methylbenzoyl) methyl phenyl sulfimide (4ha)}<smiles>Cc1cccc(C(=O)N(C)S(C)(=O)=Nc2ccccc2)c1</smiles>

Following typical experiment 3.1, 3-(3-methylphenyl)-1,4,2-dioxazol-5one $1 \mathbf{h}(23.0 \mathrm{mg}, 0.13 \mathrm{mmol})$ and methyl phenyl sulfide 3a $(11.8 \mu \mathrm{L}, 0.1$ mmol), after chromatography ( $n$-hexane/AcOEt $=1 / 1$ to $3 / 7$ ) the corresponding $N$-benzoyl sulfimide 4 ha was obtained as white solid (24.8 mg, > 99\%).; The enantiomeric excess of the sulfimide 4 ha was determined to be $97 \%$ ee by HPLC analysis using a chiral stationary phase [DAICEL CHIRALPAK IF-3 (Hexane $/ i$-PrOH $=6 / 4,0.5 \mathrm{~mL} / \mathrm{min}), t_{\mathrm{r}}($ Major $)=19.0 \mathrm{~min}, t_{\mathrm{r}}($ Minor $\left.)=16.1 \mathrm{~min}\right]$; white solid; $[\alpha]_{\mathrm{D}}{ }^{25}=-140.8$ $\left(c=1.20, \mathrm{CHCl}_{3}\right) ;{ }^{1} \mathrm{H}$ NMR $\left(400 \mathrm{MHz}, \mathrm{CDCl}_{3}\right) \delta 8.00(\mathrm{~s}, 1 \mathrm{H}), 7.97(\mathrm{~d}, J=6.8 \mathrm{~Hz}, 1 \mathrm{H}), 7.85-$ $7.82(\mathrm{~m}, 2 \mathrm{H}), 7.55-7.51(\mathrm{~m}, 3 \mathrm{H}), 7.29(\mathrm{~d}, J=7.8 \mathrm{~Hz}, 1 \mathrm{H}), 2.92(\mathrm{~s}, 3 \mathrm{H}), 2.39(\mathrm{~s}, 3 \mathrm{H}) \mathrm{ppm} ;{ }^{13} \mathrm{C}$ NMR $\left(100 \mathrm{MHz}, \mathrm{CDCl}_{3}\right) \delta 176.8,140.9,136.1,133.5,132.0,129.9,128.7,128.5,126.6$, 35.1, 21.5 ppm; FT-IR (KBr): 3057, 3021, 2920, 2860, 1593, 1445, 1323, 1281, 1213, 1113 , 1084, 974, 914, 829, 795, 746, $687 \mathrm{~cm}^{-1}$; HRESI-MS $(\mathrm{m} / \mathrm{z})\left[\mathrm{M}+\mathrm{Na}^{+}\right]$, calcd for $\left[\mathrm{C}_{15} \mathrm{H}_{15} \mathrm{NNaOS}\right]^{+} 280.0767$, found: $\mathrm{m} / \mathrm{z}=280.0767$.

\section{9. (-)- $N$-(3-Bromobenzoyl) methyl phenyl sulfimide (4ia)}<smiles></smiles>

Following typical experiment 3.1, 3-(3-bromophenyl)-1,4,2-dioxazol-5one $1 \mathbf{i}(31.3 \mathrm{mg}, 0.13 \mathrm{mmol})$ and methyl phenyl sulfide $\mathbf{3 a}(11.8 \mu \mathrm{L}, 0.1$ mmol), after chromatography ( $n$-hexane/AcOEt $=1 / 1$ to $3 / 7)$ the corresponding $N$-benzoyl sulfimide 4ia was obtained as white solid (32.0 $\mathrm{mg},>99 \%)$; The enantiomeric excess of the sulfimide 4ia was determined to be $92 \%$ ee by HPLC analysis using a chiral stationary phase [DAICEL CHIRALPAK IF-3 (Hexane $/ i-\mathrm{PrOH}=6 / 4,0.5 \mathrm{~mL} / \mathrm{min}), t_{\mathrm{r}}($ Major $)=20.8 \mathrm{~min}, t_{\mathrm{r}}($ Minor $)=$ $15.4 \mathrm{~min}]$; white solid; $[\alpha]_{\mathrm{D}}{ }^{25}=-89.8\left(c=1.35, \mathrm{CHCl}_{3}\right) ;{ }^{1} \mathrm{H}$ NMR $\left(400 \mathrm{MHz}, \mathrm{CDCl}_{3}\right) \delta 8.33$ (pseud-t, $J=1.7 \mathrm{~Hz}, 1 \mathrm{H}), 8.07(\mathrm{~d}, J=7.8 \mathrm{~Hz}, 1 \mathrm{H}), 7.83(\mathrm{dd}, J=7.6,2.2 \mathrm{~Hz}, 2 \mathrm{H}), 7.57-7.53$ (m, 4H), 7.25 (pseud-t, $J=7.8 \mathrm{~Hz}, 1 \mathrm{H}), 2.92$ (s, 3H) ppm; ${ }^{13} \mathrm{C}$ NMR $\left(100 \mathrm{MHz}, \mathrm{CDCl}_{3}\right) \delta$ 175.1, 138.4, 135.5, 133.6, 132.3, 131.8, 130.0, 129.4, 127.2, 126.7, 122.0, 35.1 ppm; FT-IR (KBr): 3539, 3445, 3065, 1626, 1587, 1558, 1506, 1450, 1395, 1248, 1206, 1150, 1099, 993 , $881,853,800,748 \mathrm{~cm}^{-1}$; HRESI-MS $(\mathrm{m} / \mathrm{z})\left[\mathrm{M}+\mathrm{Na}^{+}\right]$, calcd for $\left[\mathrm{C}_{14} \mathrm{H}_{12} \mathrm{BrNNaOS}\right]^{+} 343.9715$, found: $\mathrm{m} / \mathrm{z}=343.9719$. 


\subsection{0. (-)- $N$-(3-Chlorobenzoyl) methyl phenyl sulfimide (4ja)}<smiles></smiles>

Following typical experiment 3.1, 3-(3-chlorophenyl)-1,4,2-dioxazol-5one $\mathbf{1 j}$ (25.6 mg, $0.13 \mathrm{mmol})$ and methyl phenyl sulfide 3a $(11.8 \mu \mathrm{L}, 0.1$ mmol), after chromatography ( $n$-hexane/AcOEt $=1 / 1$ to $3 / 7)$ the corresponding $N$-benzoyl sulfimide $\mathbf{4 j a}$ was obtained as white solid $(27.8$ $\mathrm{mg},>99 \%$ ).; The enantiomeric excess of the sulfimide $\mathbf{4 j a}$ was determined to be $92 \%$ ee by HPLC analysis using a chiral stationary phase [DAICEL CHIRALPAK IF$3($ Hexane $/ i-\mathrm{PrOH}=6 / 4,0.5 \mathrm{~mL} / \mathrm{min}), t_{\mathrm{r}}($ Major $)=19.0 \mathrm{~min}, t_{\mathrm{r}}$ (Minor) $\left.=14.7 \mathrm{~min}\right]$; white solid; $[\alpha]_{\mathrm{D}}{ }^{25}=-108.5\left(c=1.26, \mathrm{CHCl}_{3}\right) ;{ }^{1} \mathrm{H}$ NMR $\left(400 \mathrm{MHz}, \mathrm{CDCl}_{3}\right) \delta 8.17$ (pseud-t, $J=1.7$ $\mathrm{Hz}, 1 \mathrm{H}), 8.03(\mathrm{dd}, J=7.8,1.2 \mathrm{~Hz}, 1 \mathrm{H}), 7.83(\mathrm{dd}, J=7.6,2.2 \mathrm{~Hz}, 2 \mathrm{H}), 7.58-7.53(\mathrm{~m}, 3 \mathrm{H})$, 7.42-7.39 (m, 1H), 7.31 (pseud-t, $J=7.6 \mathrm{~Hz}, 1 \mathrm{H}), 2.93(\mathrm{~s}, 3 \mathrm{H}) \mathrm{ppm} ;{ }^{13} \mathrm{C}$ NMR $(100 \mathrm{MHz}$, $\left.\mathrm{CDCl}_{3}\right) \delta 175.3,138.2,135.5,133.8,132.3,130.7,130.0,129.1,128.9,126.8,126.7,35.1$ ppm; FT-IR (KBr): 3063, 3011, 2922, 1674, 1591, 1553, 1476, 1416, 1314, 1260, 1146, 1076, $970,895,812,748,683 \mathrm{~cm}^{-1}$; HRESI-MS $(\mathrm{m} / \mathrm{z})\left[\mathrm{M}+\mathrm{Na}^{+}\right]$, calcd for $\left[\mathrm{C}_{14} \mathrm{H}_{12} \mathrm{CINNaOS}\right]^{+}$ 300.0220 , found: $\mathrm{m} / \mathrm{z}=300.0221$.

\subsection{1. (-)- $N$-(3-Fluorobenzoyl) methyl phenyl sulfimide (4ka)}<smiles>CN(C(=O)c1cccc(F)c1)[As](C)N(C)c1ccccc1</smiles>

Following typical experiment 3.1, 3-(3-fluorophenyl)-1,4,2-dioxazol-5one $1 \mathbf{k}(23.5 \mathrm{mg}, 0.13 \mathrm{mmol})$ and methyl phenyl sulfide 3a $(11.8 \mu \mathrm{L}, 0.1$ mmol), after chromatography ( $n$-hexane/AcOEt $=1 / 1$ to $3 / 7$ ) the corresponding $N$-benzoyl sulfimide $\mathbf{4 k a}$ was obtained as white solid $(25.6$ mg, 98\%).; The enantiomeric excess of the sulfimide $4 \mathbf{k a}$ was determined to be $93 \%$ ee by HPLC analysis using a chiral stationary phase [DAICEL CHIRALPAK IF$3($ Hexane $/ i-\mathrm{PrOH}=6 / 4,0.5 \mathrm{~mL} / \mathrm{min}), t_{\mathrm{r}}$ (Major) $=16.6 \mathrm{~min}, t_{\mathrm{r}}$ (Minor) $\left.=14.1 \mathrm{~min}\right]$; white solid; $[\alpha]_{\mathrm{D}}{ }^{25}=-129.6\left(c=1.14, \mathrm{CHCl}_{3}\right) ;{ }^{1} \mathrm{H}$ NMR $\left(400 \mathrm{MHz}, \mathrm{CDCl}_{3}\right) \delta 7.94(\mathrm{dt}, J=7.8,1.2$ $\mathrm{Hz}, 1 \mathrm{H}), 7.88-7.82(\mathrm{~m}, 3 \mathrm{H}), 7.58-7.53(\mathrm{~m}, 3 \mathrm{H}), 7.34(\mathrm{dt}, J=7.9,5.7 \mathrm{~Hz}, 1 \mathrm{H}), 7.13$ (ddt, $J=$ 8.3, 2.9, $1.3 \mathrm{~Hz}, 1 \mathrm{H}), 2.93(\mathrm{~s}, 3 \mathrm{H}) \mathrm{ppm} ;{ }^{13} \mathrm{C} \mathrm{NMR}\left(100 \mathrm{MHz}, \mathrm{CDCl}_{3}\right) \delta 174.9(\mathrm{~d}, J=3.3 \mathrm{~Hz})$, $161.1(\mathrm{~d}, J=254 \mathrm{~Hz}), 135.4,132.2,131.6(\mathrm{~d}, J=8.3 \mathrm{~Hz}), 131.3,130.0,126.8,125.4(\mathrm{~d}, J=$ $11.6 \mathrm{~Hz}), 123.5$ (d, $J=4.1 \mathrm{~Hz}), 116.4$ (d, $J=23.2), 35.1 \mathrm{ppm}$; FT-IR $(\mathrm{KBr}): 3065,3013,2920$, $1555,1477,1433,1315,1209,1080,968,925,826,750,681 \mathrm{~cm}^{-1} ;$ HRESI-MS (m/z) [M + $\mathrm{Na}^{+}$], calcd for $\left[\mathrm{C}_{14} \mathrm{H}_{12} \mathrm{FNNaOS}\right]^{+} 284.0516$, found: $\mathrm{m} / \mathrm{z}=284.0516$.

\subsection{2. (-)-N-(2-Methylbenzoyl) methyl phenyl sulfimide (4la)}<smiles></smiles>

Following typical experiment 3.1, 3-(2-methylphenyl)-1,4,2-dioxazol-5-one $11(23.0 \mathrm{mg}, 0.13 \mathrm{mmol})$ and methyl phenyl sulfide $3 \mathbf{a}(11.8 \mu \mathrm{L}, 0.1 \mathrm{mmol})$, after chromatography $(n$-hexane/AcOEt $=1 / 1$ to $3 / 7)$ the corresponding $N$ benzoyl sulfimide 4la was obtained as white solid (11.9 mg, 46\%).; Colorless crystals were obtained from $\mathrm{CHCl}_{3} / \mathrm{Hexane}$; m.p. 127.5-127.6 ${ }^{\circ} \mathrm{C}$.; The enantiomeric excess of the sulfimide 4la was determined to be $90 \%$ ee by HPLC analysis using a chiral stationary phase [DAICEL CHIRALPAK IF-3 (Hexane $/ i$-PrOH $=6 / 4,0.5$ $\mathrm{mL} / \mathrm{min}), t_{\mathrm{r}}($ Major $)=19.5 \mathrm{~min}, t_{\mathrm{r}}($ Minor $\left.)=15.6 \mathrm{~min}\right]$; white solid; $[\alpha]_{\mathrm{D}}^{25}=-163.3(c=0.82$, $\left.\mathrm{CHCl}_{3}\right) ;{ }^{1} \mathrm{H}$ NMR $\left(400 \mathrm{MHz}, \mathrm{CDCl}_{3}\right) \delta$ 7.88-7.84 (m, 3H), 7.60-7.56 (m, 3H), 7.29-7.18 (m, 4H), $2.94(\mathrm{~s}, 3 \mathrm{H}), 2.57(\mathrm{~s}, 3 \mathrm{H}) \mathrm{ppm} ;{ }^{13} \mathrm{C} \mathrm{NMR}\left(100 \mathrm{MHz}, \mathrm{CDCl}_{3}\right) \delta 180.0,137.1,135.9$, 132.2, 130.9, 130.0, 129.3, 129.1, 126.7, 125.3, 34.9, 21.1 ppm; FT-IR (KBr): 3057, 3013, 2961, 2922, 2359, 1552, 1476, 1439, 1319, 1277, 1146, 1092, 968, 876, 814, 745, 689 $\mathrm{cm}^{-1}$; HRESI-MS $(\mathrm{m} / \mathrm{z})\left[\mathrm{M}+\mathrm{Na}^{+}\right]$, calcd for $\left[\mathrm{C}_{15} \mathrm{H}_{15} \mathrm{NNaOS}\right]^{+} 280.0767$, found: $\mathrm{m} / \mathrm{z}=280.0768$. 


\subsection{3. (-)-N-(2-Bromobenzoyl) methyl phenyl sulfimide (4ma)}<smiles>C[Sb](C)(=S)NC(=O)c1ccccc1Br</smiles>

Following typical experiment 3.1, 3-(2-bromophenyl)-1,4,2-dioxazol-5-one $1 \mathbf{m}(31.3 \mathrm{mg}, 0.13 \mathrm{mmol})$ and methyl phenyl sulfide $3 \mathbf{a}(11.8 \mu \mathrm{L}, 0.1 \mathrm{mmol})$, after chromatography ( $n$-hexane/AcOEt $=1 / 1$ to $3 / 7)$ the corresponding $N$ benzoyl sulfimide $4 \mathrm{ma}$ was obtained as white solid $(24.1 \mathrm{mg}, 75 \%)$; The enantiomeric excess of the sulfimide $4 \mathrm{ma}$ was determined to be $94 \%$ ee by HPLC analysis using a chiral stationary phase [DAICEL CHIRALPAK IF-3 (Hexane/ $i$-PrOH $=6 / 4,0.5 \mathrm{~mL} / \mathrm{min}), t_{\mathrm{r}}($ Major $)=22.9 \mathrm{~min}, t_{\mathrm{r}}($ Minor $\left.)=19.0 \mathrm{~min}\right]$; white solid; $[\alpha]_{\mathrm{D}}{ }^{25}=-143.9$ $\left(c=0.95, \mathrm{CHCl}_{3}\right),{ }_{1}^{1} \mathrm{H}$ NMR $\left(400 \mathrm{MHz}, \mathrm{CDCl}_{3}\right) \delta 7.88(\mathrm{dd}, J=7.6,2.2 \mathrm{~Hz}, 2 \mathrm{H}), 7.63(\mathrm{dd}, J$ $=7.6,1.7 \mathrm{~Hz}, 1 \mathrm{H}), 7.58-7.54(\mathrm{~m}, 4 \mathrm{H}), 7.39(\mathrm{dt}, J=7.4,1.1 \mathrm{~Hz}, 1 \mathrm{H}), 7.17(\mathrm{dt}, J=7.7,1.7 \mathrm{~Hz}$, 1H), $2.94(\mathrm{~s}, 3 \mathrm{H}) \mathrm{ppm} ;{ }^{13} \mathrm{C}$ NMR $\left(100 \mathrm{MHz}, \mathrm{CDCl}_{3}\right) \delta 178.0,139.8,135.0,133.2,132.4$, 130.0, 129.7, 127.0, 120.2, 34.8 ppm; FT-IR (KBr): 3539, 3445, 3065, 1626, 1587, 1558, 1506, 1450, 1395, 1248, 1206, 1150, 1099, 993, 881, 853, 800, $748 \mathrm{~cm}^{-1}$; HRESI-MS (m/z) $\left[\mathrm{M}+\mathrm{Na}^{+}\right]$, calcd for $\left[\mathrm{C}_{14} \mathrm{H}_{12} \mathrm{BrNNaOS}\right]^{+} 343.9715$, found: $\mathrm{m} / \mathrm{z}=345.9715$.

\subsection{4. (-)- $N$-(2-Chlorobenzoyl) methyl phenyl sulfimide (4na)}<smiles>CN(C(=O)c1ccccc1Cl)[SH](C)(=S)Nc1ccccc1</smiles>

Following typical experiment 3.1, 3-(2-chlorophenyl)-1,4,2-dioxazol-5-one 1n $(25.6 \mathrm{mg}, 0.13 \mathrm{mmol})$ and methyl phenyl sulfide $3 \mathrm{a}(11.8 \mu \mathrm{L}, 0.1 \mathrm{mmol})$, after chromatography $(n$-hexane/AcOEt $=1 / 1$ to $3 / 7)$ the corresponding $N$ benzoyl sulfimide 4na was obtained as white solid (23.9 mg, 86\%).; Colorless crystals were obtained from $\mathrm{CHCl}_{3} / \mathrm{Hexane}$; m.p. 126.6-126.7 ${ }^{\circ} \mathrm{C}$.; The enantiomeric excess of the sulfimide 4na was determined to be $94 \%$ ee by HPLC analysis using a chiral stationary phase [DAICEL CHIRALPAK IF-3 (Hexane $/ i$-PrOH $=6 / 4,0.5$ $\mathrm{mL} / \mathrm{min}), t_{\mathrm{r}}($ Major $)=22.2 \mathrm{~min}, t_{\mathrm{r}}($ Minor $\left.)=18.7 \mathrm{~min}\right]$; white solid; $[\alpha]_{\mathrm{D}}{ }^{25}=-187.0(c=1.17$, $\left.\mathrm{CHCl}_{3}\right) ;{ }^{1} \mathrm{H}$ NMR $\left(400 \mathrm{MHz}, \mathrm{CDCl}_{3}\right) \delta$ 7.89-7.86 (m, 2H), 7.76-7.63 (m, 1H), 7.62-7.50 (m, $3 \mathrm{H}), 7.44-7.33(\mathrm{~m}, 1 \mathrm{H}), 7.32-7.11(\mathrm{~m}, 2 \mathrm{H}), 2.94(\mathrm{~s}, 3 \mathrm{H}) \mathrm{ppm} ;{ }^{13} \mathrm{C} \mathrm{NMR}\left(100 \mathrm{MHz}, \mathrm{CDCl}_{3}\right)$ $\delta 177.3,137.6,135.1,132.4,131.7,130.1,130.0,129.9,129.8,126.9,126.4,34.9$ ppm; FTIR (KBr): 3061, 3011, 1580, 1476, 1433, 1319, 1150, 1043, 970, 874, 799, 748, 692, 646, $552 \mathrm{~cm}^{-1}$; HRESI-MS $(\mathrm{m} / \mathrm{z})\left[\mathrm{M}+\mathrm{Na}^{+}\right]$, calcd for $\left[\mathrm{C}_{14} \mathrm{H}_{12} \mathrm{ClNNaOS}\right]^{+} 300.0220$, found: $\mathrm{m} / \mathrm{z}$ $=300.0220$.

\subsection{5. (-)- $N$-(2-Fluorobenzoyl) methyl phenyl sulfimide (40a)}<smiles>C/C=S(/C)N(C)C(=O)c1ccccc1F</smiles>

Following typical experiment 3.1, 3-(2-fluorophenyl)-1,4,2-dioxazol-5-one $10(23.5 \mathrm{mg}, 0.13 \mathrm{mmol})$ and methyl phenyl sulfide $3 \mathbf{a}(11.8 \mu \mathrm{L}, 0.1 \mathrm{mmol})$, after chromatography $(n$-hexane/AcOEt $=1 / 1$ to $3 / 7)$ the corresponding $N$ benzoyl sulfimide $40 a$ was obtained as white solid $(26.1 \mathrm{mg},>99 \%)$; The enantiomeric excess of the sulfimide $40 a$ was determined to be $95 \%$ ee by HPLC analysis using a chiral stationary phase[DAICEL CHIRALPAK IF-3 (Hexane $/ i$-PrOH $=6 / 4,0.5 \mathrm{~mL} / \mathrm{min}), t_{\mathrm{r}}($ Major $)=20.2 \mathrm{~min}, t_{\mathrm{r}}($ Minor $\left.)=16.8 \mathrm{~min}\right]$; white solid; $[\alpha]_{\mathrm{D}}{ }^{25}=-133.7$ $\left(c=1.20, \mathrm{CHCl}_{3}\right) ;{ }^{1} \mathrm{H} \mathrm{NMR}\left(400 \mathrm{MHz}, \mathrm{CDCl}_{3}\right) \delta 7.92(\mathrm{dt}, J=7.6,2.0 \mathrm{~Hz}, 1 \mathrm{H}), 7.89-7.85$ $(\mathrm{m}, 1 \mathrm{H}), 7.59-7.54(\mathrm{~m}, 3 \mathrm{H}), 7.40-7.34(\mathrm{~m}, 1 \mathrm{H}), 7.14(\mathrm{dt}, J=7.6,1.0 \mathrm{~Hz}, 1 \mathrm{H}), 7.08$ (ddd, $J=$ $10.2,8.3,1.0 \mathrm{~Hz}, 1 \mathrm{H}), 2.95$ (s, 3H) ppm; ${ }^{13} \mathrm{C} \mathrm{NMR}\left(100 \mathrm{MHz}, \mathrm{CDCl}_{3}\right) \delta 174.9(\mathrm{~d}, J=3.3$ $\mathrm{Hz}), 161.1(\mathrm{~d}, J=254.1 \mathrm{~Hz}), 135.4,132.2,131.6(\mathrm{~d}, 8.3 \mathrm{~Hz}), 131.3,130.0,126.8,125.4(\mathrm{~d}$, $J=11.6 \mathrm{~Hz}), 123.5(\mathrm{~d}, J=4.1 \mathrm{~Hz}), 116.4(\mathrm{~d}, J=23.2 \mathrm{~Hz}), 35.1 \mathrm{ppm}$; FT-IR (KBr): 3539 , $3445,3065,1626,1587,1558,1506,1450,1395,1248,1206,1150,1099,993,881,853,800$, 
$748 \mathrm{~cm}^{-1}$; HRESI-MS $(\mathrm{m} / \mathrm{z})\left[\mathrm{M}+\mathrm{Na}^{+}\right]$, calcd for $\left[\mathrm{C}_{14} \mathrm{H}_{12} \mathrm{FNNaOS}\right]^{+} 284.0516$, found: $\mathrm{m} / \mathrm{z}=$ 284.0517 .

\subsection{6. (R)-(-)- $N$-Acetyl methyl phenyl sulfimide (4pa)}

Following typical experiment 3.1, 3-methyl-1,4,2-dioxazol-5-one 1p $(13.3 \mathrm{mg}$,
0.13 mmol) and methyl phenyl sulfide 3a $(11.8 \mu \mathrm{L}, 0.1 \mathrm{mmol})$, after
chromatography ( $n$-hexane/AcOEt $=1 / 1$ to $3 / 7)$ the corresponding $N$-acetyl
sulfimide 4pa was obtained as white solid $(16.4 \mathrm{mg}, 91 \%)$; The enantiomeric excess of the sulfimide 4pa was determined to be $94 \%$ ee by HPLC analysis using a chiral stationary phase [DAICEL CHIRALCEL OZ-3 (Hexane $/ \mathrm{EtOH}=6 / 4,0.5 \mathrm{~mL} / \mathrm{min}), t_{\mathrm{r}}$ (Major) $=22.6 \mathrm{~min}, t_{\mathrm{r}}($ Minor $\left.)=17.6 \mathrm{~min}\right]$; white solid; $[\alpha]_{\mathrm{D}}{ }^{25}=-300.2\left(c=0.80, \mathrm{CHCl}_{3}\right) ;{ }^{1} \mathrm{H} \mathrm{NMR}$ $\left(400 \mathrm{MHz}, \mathrm{CDCl}_{3}\right) \delta 7.75(\mathrm{dd}, J=7.8,2.4 \mathrm{~Hz}, 2 \mathrm{H}), 7.56-7.51(\mathrm{~m}, 3 \mathrm{H}), 2.81(\mathrm{~s}, 3 \mathrm{H}), 2.13(\mathrm{~s}$, 3H) ppm; ${ }^{13} \mathrm{C}$ NMR $\left(100 \mathrm{MHz}, \mathrm{CDCl}_{3}\right) \delta 182.1,135.6,132.2,129.9,126.7,34.5,24.2 \mathrm{ppm}$; FT-IR (KBr): 3539, 3445, 3065, 1626, 1587, 1558, 1506, 1450, 1395, 1248, 1206, 1150, 1099 , 993, 881, 853, 800, $748 \mathrm{~cm}^{-1}$; HRESI-MS $(\mathrm{m} / \mathrm{z})\left[\mathrm{M}+\mathrm{Na}^{+}\right]$, calcd for $\left[\mathrm{C}_{9} \mathrm{H}_{11} \mathrm{NNaOS}\right]^{+}$ 204.0454, found: $\mathrm{m} / \mathrm{z}=204.0454$.

\subsection{7. (-)- $N$-Hexanoyl methyl phenyl sulfimide (4qa)}<smiles>CCCCCC(=O)N(C)S(C)=[SH](=O)c1ccccc1</smiles>

Following typical experiment 3.1, 3- $n$-pentyl-1,4,2-dioxazol-5-one 1q (20.4 $\mathrm{mg}, 0.13 \mathrm{mmol})$ and methyl phenyl sulfide 3a $(11.8 \mu \mathrm{L}, 0.1 \mathrm{mmol})$, after chromatography ( $n$-hexane/AcOEt $=1 / 1$ to $3 / 7$ ) the corresponding $N$ hexanoyl sulfimide 4qa was obtained as white solid $(23.7 \mathrm{mg}$, > 99\%).; The enantiomeric excess of the sulfimide $\mathbf{4 q a}$ was determined to be $93 \%$ ee by HPLC analysis using a chiral stationary phase [DAICEL CHIRALCEL OZ-3 (Hexane/i$\mathrm{PrOH}=2 / 8,0.5 \mathrm{~mL} / \mathrm{min}), t_{\mathrm{r}}($ Major $)=19.8 \mathrm{~min}, t_{\mathrm{r}}($ Minor $\left.)=10.9 \mathrm{~min}\right]$; white solid; $[\alpha]_{\mathrm{D}}{ }^{25}=$ $-254.5\left(c=1.06, \mathrm{CHCl}_{3}\right) ;{ }^{1} \mathrm{H}$ NMR $\left(400 \mathrm{MHz}, \mathrm{CDCl}_{3}\right) \delta 8.09(\mathrm{~d}, J=7.3 \mathrm{~Hz}, 2 \mathrm{H}), 7.43-7.35$ $(\mathrm{m}, 3 \mathrm{H}), 3.18(\mathrm{dt}, J=12.4,8.0 \mathrm{~Hz}, 1 \mathrm{H}), 2.93(\mathrm{dt}, J=12.4,7.6 \mathrm{~Hz}, 1 \mathrm{H}), 2.73(\mathrm{~s}, 3 \mathrm{H}), 1.84-$ $1.77(\mathrm{~m}, 2 \mathrm{H}), 1.52-1.44(\mathrm{~m}, 2 \mathrm{H}), 1.35-1.27(\mathrm{~m}, 8 \mathrm{H}), 0.88(\mathrm{t}, J=7.3 \mathrm{~Hz}, 3 \mathrm{H}) \mathrm{ppm} ;{ }^{13} \mathrm{C} \mathrm{NMR}$ $\left(100 \mathrm{MHz}, \mathrm{CDCl}_{3}\right) \delta 177.2,136.6,130.6,128.6,127.8,46.7,31.7,30.0,29.0,29.0,28.6,23.2$, 22.6, 14.0 ppm; FT-IR (KBr): 3539, 3445, 3065, 1626, 1587, 1558, 1506, 1450, 1395, 1248, $1206,1150,1099,993,881,853,800,748 \mathrm{~cm}^{-1}$; HRESI-MS $(\mathrm{m} / \mathrm{z})\left[\mathrm{M}+\mathrm{Na}^{+}\right]$, calcd for $\left[\mathrm{C}_{13} \mathrm{H}_{19} \mathrm{NNaOS}\right]^{+} 260.1080$, found: $\mathrm{m} / \mathrm{z}=260.1080$.

\subsection{8. (-)- $N$-Isobutyloyl methyl phenyl sulfimide (4ra)}<smiles>CC(C)C(=O)N(C)S(C)(C)c1ccccc1</smiles>

Following typical experiment 3.1, 3-i-propyl-1,4,2-dioxazol-5-one 1r (16.8 mg, $0.13 \mathrm{mmol})$ and methyl phenyl sulfide 3a $(11.8 \mu \mathrm{L}, 0.1 \mathrm{mmol})$, after chromatography ( $n$-hexane/AcOEt $=1 / 1$ to $3 / 7)$ the corresponding $N$ isobutyloyl sulfimide 4ra was obtained as white solid (10.9 mg, 52\%).; The enantiomeric excess of the sulfimide 4ra was determined to be $96 \%$ ee by HPLC analysis using a chiral stationary phase [DAICEL CHIRALCEL OZ-3 (Hexane $i$ $\operatorname{PrOH}=2 / 8,0.5 \mathrm{~mL} / \mathrm{min}), t_{\mathrm{r}}($ Major $)=13.1 \mathrm{~min}, t_{\mathrm{r}}($ Minor $\left.)=10.0 \mathrm{~min}\right]$; white solid; $[\alpha]_{\mathrm{D}}{ }^{25}=$ -293.7 $\left(c=0.44, \mathrm{CHCl}_{3}\right) ;{ }^{1} \mathrm{H}$ NMR $\left(400 \mathrm{MHz}, \mathrm{CDCl}_{3}\right) \delta$ 7.76-7.73 $(\mathrm{m}, 2 \mathrm{H}), 7.55-7.50(\mathrm{~m}$, $3 \mathrm{H}), 2.81(\mathrm{~s}, 3 \mathrm{H}), 2.72-2.65(\mathrm{~m}, 1 \mathrm{H}), 1.18(\mathrm{t}, J=6.8 \mathrm{~Hz}, 6 \mathrm{H}) \mathrm{ppm} ;{ }^{13} \mathrm{C}$ NMR $(100 \mathrm{MHz}$, $\left.\mathrm{CDCl}_{3}\right) \delta 188.7,136.1,132.0,129.9,126.5,36.2,34.4,20.6,20.3$ ppm; FT-IR (KBr): 3539, $3445,3065,1626,1587,1558,1506,1450,1395,1248,1206,1150,1099,993,881,853,800$, 
$748 \mathrm{~cm}^{-1}$; HRESI-MS $(\mathrm{m} / \mathrm{z})\left[\mathrm{M}+\mathrm{Na}^{+}\right]$, calcd for $\left[\mathrm{C}_{11} \mathrm{H}_{15} \mathrm{NNaOS}\right]^{+} 232.0767$, found: $\mathrm{m} / \mathrm{z}=$ 232.0768.

\subsection{9. (-)-(E)- $N$-Cinnamoyl methyl phenyl sulfimide (4ta)}<smiles>CN(C(=O)/C=C/c1ccccc1)[SH](C)(C)=S</smiles>

Following typical experiment 3.1, (E)-3-cinnamyl-1,4,2-dioxazol-5-one 1t (26.4 $\mathrm{mg}, 0.13 \mathrm{mmol})$ and methyl phenyl sulfide $3 \mathbf{a}(11.8 \mu \mathrm{L}, 0.1 \mathrm{mmol})$, after chromatography ( $n$-hexane/AcOEt $=1 / 1$ to $3 / 7)$ the corresponding $N$ isobutyloyl sulfimide 4 ta was obtained as white solid (25.3 $\mathrm{mg}, 94 \%)$; The enantiomeric excess of the sulfimide 4 ta was determined to be $94 \%$ ee by HPLC analysis using a chiral stationary phase [DAICEL CHIRALPAK IF-3 (Hexane/ $i$ $\mathrm{PrOH}=6 / 4,0.5 \mathrm{~mL} / \mathrm{min}$ ), $t_{\mathrm{r}}$ (Major) $=31.5 \mathrm{~min}, t_{\mathrm{r}}($ Minor $\left.)=24.0 \mathrm{~min}\right]$; white solid; $[\alpha]_{\mathrm{D}}{ }^{25}=$ $-168.4\left(c=0.93, \mathrm{CHCl}_{3}\right) ;{ }^{1} \mathrm{H} \mathrm{NMR}\left(400 \mathrm{MHz}, \mathrm{CDCl}_{3}\right) \delta 7.81(\mathrm{dd}, J=7.3,2.4 \mathrm{~Hz}, 2 \mathrm{H}), 7.59$ $(\mathrm{d}, J=16.1 \mathrm{~Hz}, 1 \mathrm{H}), 7.56-7.52(\mathrm{~m}, 5 \mathrm{H}), 7.36-7.30(\mathrm{~m}, 3 \mathrm{H}), 6.79(\mathrm{~d}, J=16.1 \mathrm{~Hz}, 1 \mathrm{H}), 2.90$ (s, 3H) ppm; ${ }^{13} \mathrm{C}$ NMR $\left(100 \mathrm{MHz}, \mathrm{CDCl}_{3}\right) \delta 176.8,139.6,135.7,135.6,132.2,130.0,129.0$, 128.6, 127.7, 126.7, 124.2, 34.6 ppm; FT-IR (KBr): 3539, 3445, 3065, 1626, 1587, 1558, 1506, 1450, 1395, 1248, 1206, 1150, 1099, 993, 881, 853, 800, $748 \mathrm{~cm}^{-1}$; HRESI-MS (m/z) $\left[\mathrm{M}+\mathrm{Na}^{+}\right]$, calcd for $\left[\mathrm{C}_{16} \mathrm{H}_{15} \mathrm{NNaOS}\right]^{+} 292.0767$, found: $\mathrm{m} / \mathrm{z}=292.0766$.

\subsection{0. (-)- $N$-Benzoyl methyl 4-methylphenyl sulfimide (4ab)}

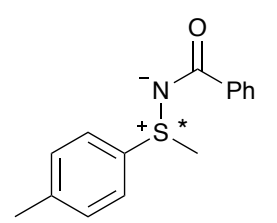

Following typical experiment 3.1, 3-phenyl-1,4,2-dioxazol-5-one 1a (21.2 $\mathrm{mg}, 0.13 \mathrm{mmol})$ and methyl 4-methylphenyl sulfide $3 \mathbf{b}(13.5 \mu \mathrm{L}, 0.1$ mmol), after chromatography ( $n$-hexane/AcOEt $=1 / 1$ to $3 / 7$ ) the corresponding $N$-benzoyl sulfimide 4 ab was obtained as white solid (25.7 $\mathrm{mg},>$ 99\%).; The enantiomeric excess of the sulfimide 4ab was determined to be $95 \%$ ee by HPLC analysis using a chiral stationary phase

[DAICEL CHIRALPAK IF-3 (Hexane $/ i-\mathrm{PrOH}=6 / 4,0.5 \mathrm{~mL} / \mathrm{min}), t_{\mathrm{r}}$ (Major) $=21.9 \mathrm{~min}, t_{\mathrm{r}}$ $($ Minor $)=16.9 \mathrm{~min}]$; white solid; $[\alpha]_{\mathrm{D}}{ }^{25}=-141.7\left(c=1.19, \mathrm{CHCl}_{3}\right) ;{ }^{1} \mathrm{H}$ NMR $(400 \mathrm{MHz}$, $\left.\mathrm{CDCl}_{3}\right) \delta 8.16(\mathrm{~d}, J=6.8 \mathrm{~Hz}, 1 \mathrm{H}), 7.73(\mathrm{~d}, J=8.3 \mathrm{~Hz}, 1 \mathrm{H}), 7.45-7.33(\mathrm{~m}, 5 \mathrm{H}), 2.90(\mathrm{~s}, 3 \mathrm{H})$, $2.41(\mathrm{~s}, 3 \mathrm{H}) \mathrm{ppm} ;{ }^{13} \mathrm{C}$ NMR $\left(100 \mathrm{MHz}, \mathrm{CDCl}_{3}\right) \delta 176.6,142.9,136.2,132.4,130.6,128.7$, 126.6, 35.1, 21.3 ppm; FT-IR (KBr): 3053, 2922, 2866, 2359, 1597, 1552, 1493, 1412, 1329 , $1171,1128,1074,1024,966,870,806,716,621 \mathrm{~cm}^{-1}$; HRESI-MS $(\mathrm{m} / \mathrm{z})\left[\mathrm{M}+\mathrm{Na}^{+}\right]$, calcd for $\left[\mathrm{C}_{15} \mathrm{H}_{15} \mathrm{NNaOS}\right]^{+} 280.0767$, found: $\mathrm{m} / \mathrm{z}=280.0767$.

\subsection{1. (-)- $N$-Benzoyl 4-methoxyphenyl methyl sulfimide (4ac)}

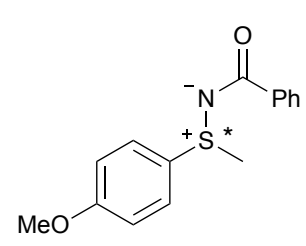

Following typical experiment 3.1, 3-phenyl-1,4,2-dioxazol-5-one 1a $(21.2 \mathrm{mg}, 0.13 \mathrm{mmol})$ and 4-methoxyphenyl methyl sulfide $\mathbf{3 c}(13.9 \mu \mathrm{L}$, $0.1 \mathrm{mmol})$, after chromatography $(n$-hexane/AcOEt $=1 / 1$ to $3 / 7)$ the corresponding $N$-benzoyl sulfimide 4 ac was obtained as white solid $(27.3 \mathrm{mg},>99 \%)$; The enantiomeric excess of the sulfimide 4 ac was determined to be $94 \%$ ee by HPLC analysis using a chiral stationary phase [DAICEL CHIRALPAK IF-3 (Hexane $/ i-\mathrm{PrOH}=6 / 4,0.5 \mathrm{~mL} / \mathrm{min}), t_{\mathrm{r}}$ (Major) $=27.3$ min, $t_{\mathrm{r}}($ Minor $\left.)=19.9 \mathrm{~min}\right]$; white solid; $[\alpha]_{\mathrm{D}}{ }^{25}=-121.9\left(c=1.25, \mathrm{CHCl}_{3}\right) ;{ }^{1} \mathrm{H}$ NMR $(400$ $\left.\mathrm{MHz}, \mathrm{CDCl}_{3}\right) \delta 8.14(\mathrm{dt}, J=6.5,1.7 \mathrm{~Hz}, 2 \mathrm{H}), 7.78(\mathrm{dt}, J=9.4,2.4 \mathrm{~Hz}, 2 \mathrm{H}), 7.44-7.34(\mathrm{~m}$, $3 \mathrm{H}), 7.03(\mathrm{dt}, J=6.1,3.4 \mathrm{~Hz}, 2 \mathrm{H}), 3.84(\mathrm{~s}, 3 \mathrm{H}), 2.89(\mathrm{~s}, 3 \mathrm{H}) \mathrm{ppm} ;{ }^{13} \mathrm{C}$ NMR $(100 \mathrm{MHz}$, $\left.\mathrm{CDCl}_{3}\right) \delta 176.5,162.7,136.5,130.6,128.8,128.6,127.8,126.4,115.4,55.6,35.2 \mathrm{ppm}$; FTIR (KBr): 3061, 3007, 2965, 2924, 2837, 2359, 1587, 1545, 1491, 1449, 1412, 1327, 1296, 
1254, 1173, 1125, 1082, 1022, 966, 872, 826, 800, 716, $625 \mathrm{~cm}^{-1}$; HRESI-MS (m/z) [M + $\left.\mathrm{Na}^{+}\right]$, calcd for $\left[\mathrm{C}_{15} \mathrm{H}_{15} \mathrm{NNaO}_{2} \mathrm{~S}\right]^{+} 296.0716$, found: $\mathrm{m} / \mathrm{z}=296.0718$.

\subsection{2. (-)- $N$-Benzoyl 4-bromophenyl methyl sulfimide (4ad)}<smiles>C[S]1(C)=C(c2ccc(Br)cc2)c2ccccc2C1=O</smiles>

Following typical experiment 3.1, 3-phenyl-1,4,2-dioxazol-5-one 1a (21.2 $\mathrm{mg}, 0.13 \mathrm{mmol}$ ) and 4-bromophenyl methyl sulfide 3d (20.3 mg, 0.1 $\mathrm{mmol}$ ), after chromatography ( $n$-hexane/AcOEt $=1 / 1$ to $3 / 7$ ) the corresponding $N$-benzoyl sulfimide 4 ad was obtained as white solid (31.8 mg, 99\%).; Colorless crystals were obtained from $\mathrm{CHCl}_{3} / \mathrm{Hexane.;} \mathrm{m.p.}$ 106.2-106.3 ${ }^{\circ} \mathrm{C}$.; The enantiomeric excess of the sulfimide 4ad was determined to be $93 \%$ ee by HPLC analysis using a chiral stationary phase [DAICEL CHIRALPAK IF-3 (Hexane $/ i-\mathrm{PrOH}=6 / 4,0.5 \mathrm{~mL} / \mathrm{min}), t_{\mathrm{r}}($ Major $)=23.0 \mathrm{~min}, t_{\mathrm{r}}($ Minor $)=$ $15.7 \mathrm{~min}$; white solid; $[\alpha]_{\mathrm{D}}{ }^{24}=-142.1\left(c=1.52, \mathrm{CHCl}_{3}\right) ;{ }^{1} \mathrm{H}$ NMR $\left(400 \mathrm{MHz}, \mathrm{CDCl}_{3}\right) \delta 8.15$ $(\mathrm{d}, J=8.3 \mathrm{~Hz}, 2 \mathrm{H}), 7.68$ (pseudo-dd, $J=13.2,8.8 \mathrm{~Hz}, 4 \mathrm{H}$ ), 7.42 (pseudo-dt, $J=26.0,7.2$ $\mathrm{Hz}, 3 \mathrm{H}), 2.90(\mathrm{~s}, 3 \mathrm{H}) \mathrm{ppm} ;{ }^{13} \mathrm{C}$ NMR $\left(100 \mathrm{MHz}, \mathrm{CDCl}_{3}\right){ }^{13} \mathrm{C} \mathrm{NMR}\left(100 \mathrm{MHz}, \mathrm{CDCl}_{3}\right) \delta$ 176.8, 136.0, 135.1, 133.1, 130.9, 128.7, 128.1, 127.8, 126.8, 34.9 ppm; FT-IR (KBr): 3065, $3015,2922,1670,1595,1553,1476,1391,1329,1169,1126,1067,1011,970,868,814,719$ $\mathrm{cm}^{-1}$; HRESI-MS $(\mathrm{m} / \mathrm{z})\left[\mathrm{M}+\mathrm{Na}^{+}\right]$, calcd for $\left[\mathrm{C}_{14} \mathrm{H}_{12} \mathrm{BrNNaOS}\right]^{+} 343.9715$, found: $\mathrm{m} / \mathrm{z}=$ 345.9715.

\subsection{3. (-)-N-Benzoyl 4-chlorophenyl methyl sulfimide (4ae)}<smiles>C[S-](C)(C(=O)c1ccccc1)C(=O)c1ccc(Cl)cc1</smiles>

Following typical experiment 3.1, 3-phenyl-1,4,2-dioxazol-5-one 1a (21.2 mg, $0.13 \mathrm{mmol})$ and 4-chlorophenyl methyl sulfide $3 \mathbf{e}(13.0 \mu \mathrm{L}, 0.1$ mmol), after chromatography ( $n$-hexane/AcOEt $=1 / 1$ to $3 / 7$ ) the corresponding $N$-benzoyl sulfimide 4ae was obtained as white solid (27.7 $\mathrm{mg},>99 \%$ ).; The enantiomeric excess of the sulfimide 4ae was determined to be $94 \%$ ee by HPLC analysis using a chiral stationary phase [DAICEL CHIRALPAK IF-3 (Hexane $/ i-\mathrm{PrOH}=6 / 4,0.5 \mathrm{~mL} / \mathrm{min}), t_{\mathrm{r}}$ (Major) $=21.2 \mathrm{~min}, t_{\mathrm{r}}$ $($ Minor $)=15.2 \mathrm{~min}]$; white solid; $[\alpha]_{\mathrm{D}}^{25}=-161.3\left(c=1.27, \mathrm{CHCl}_{3}\right) ;{ }^{1} \mathrm{H}$ NMR $(400 \mathrm{MHz}$, $\left.\mathrm{CDCl}_{3}\right) \delta 8.16(\mathrm{~d}, J=7.3 \mathrm{~Hz}, 2 \mathrm{H}), 7.79(\mathrm{~d}, J=9.0 \mathrm{~Hz}, 2 \mathrm{H}), 7.53(\mathrm{~d}, J=8.8 \mathrm{~Hz}, 2 \mathrm{H}), 7.43$ (pseud-dt, $J=26.0,7.3 \mathrm{~Hz}, 3 \mathrm{H}), 2.91(\mathrm{~s}, 3 \mathrm{H}) \mathrm{ppm} ;{ }^{13} \mathrm{C} \mathrm{NMR}\left(100 \mathrm{MHz}, \mathrm{CDCl}_{3}\right) \delta 176.8$, 138.6, 136.0, 134.4, 130.9, 130.2, 128.7, 128.0, 127.8, 35.0 ppm; FT-IR (KBr): 3061, 3007, $2965,2924,2837,2359,1587,1545,1491,1449,1412,1327,1296,1254,1173,1125,1082$, 1022, 966, 872, 826, 800, 716, 625 $\mathrm{cm}^{-1}$; HRESI-MS $(\mathrm{m} / \mathrm{z})\left[\mathrm{M}+\mathrm{Na}^{+}\right]$, calcd for $\left[\mathrm{C}_{14} \mathrm{H}_{12} \mathrm{ClNNaOS}\right]^{+} 300.0220$, found: $\mathrm{m} / \mathrm{z}=300.0220$.

\subsection{4. (-)-N-Benzoyl 4-fluorophenyl methyl sulfimide (4af)}<smiles>C[Si](C)(NC(=O)c1ccc(F)cc1)c1ccccc1</smiles>

Following typical experiment 3.1, 3-phenyl-1,4,2-dioxazol-5-one 1a (21.2 $\mathrm{mg}, 0.13 \mathrm{mmol})$ and 4-fluorophenyl methyl sulfide $3 f(12.2 \mu \mathrm{L}, 0.1 \mathrm{mmol})$, after chromatography ( $n$-hexane/AcOEt $=1 / 1$ to $3 / 7$ ) the corresponding $N$ benzoyl sulfimide 4af was obtained as white solid (26.1 mg, >99\%).; The enantiomeric excess of the sulfimide 4af was determined to be $95 \%$ ee by HPLC analysis using a chiral stationary phase DAICEL CHIRALPAK IF$3($ Hexane $/ i-\mathrm{PrOH}=6 / 4,0.5 \mathrm{~mL} / \mathrm{min}), t_{\mathrm{r}}($ Major $)=18.6 \mathrm{~min}, t_{\mathrm{r}}($ Minor $\left.)=14.6 \mathrm{~min}\right]$; white solid; $[\alpha]_{\mathrm{D}}{ }^{25}=-133.1\left(c=1.35, \mathrm{CHCl}_{3}\right) ;{ }^{1} \mathrm{H} \mathrm{NMR}\left(400 \mathrm{MHz}, \mathrm{CDCl}_{3}\right) \delta 8.15(\mathrm{~d}, J=8.3 \mathrm{~Hz}$, 2H), $7.86(\mathrm{ddd}, J=8.8,4.9,1.5 \mathrm{~Hz}, 2 \mathrm{H}), 7.47-7.37$ (m, 3H), 7.23 (d, $J=7.8 \mathrm{~Hz}, 2 \mathrm{H}), 2.91$ (s, 
3H) ppm; ${ }^{13} \mathrm{C}$ NMR $\left(100 \mathrm{MHz}, \mathrm{CDCl}_{3}\right) \delta 176.7,164.9(\mathrm{~d}, J=254.1 \mathrm{~Hz}), 136.1,131.4(\mathrm{~d}, J$ $=2.5 \mathrm{~Hz}), 130.9,129.2(\mathrm{~d}, J=9.1 \mathrm{~Hz}), 128.7,127.8,117.3(\mathrm{~d}, J=22.3 \mathrm{~Hz}), 35.2 \mathrm{ppm}$; FTIR (KBr): 3061, 2922, 1670, 1595, 1553, 1491, 1412, 1329, 1292, 1231, 1163, 1074, 1020, 970, 829, $717 \mathrm{~cm}^{-1}$; HRESI-MS $(\mathrm{m} / \mathrm{z})\left[\mathrm{M}+\mathrm{Na}^{+}\right]$, calcd for $\left[\mathrm{C}_{14} \mathrm{H}_{12} \mathrm{FNNaOS}\right]^{+}$284.0516, found: $\mathrm{m} / \mathrm{z}=284.0516$.

\subsection{5. (-)- $N$-Benzoyl methyl 4-nitrophenyl sulfimide (4ag)}<smiles>CN(C(=O)[S+](C)(C)c1ccccc1)c1ccc([N+](=O)[O-])cc1</smiles>

Following typical experiment 3.1, 3-phenyl-1,4,2-dioxazol-5-one 1a $(21.2 \mathrm{mg}, 0.13 \mathrm{mmol})$ and 4-nitorophenyl methyl sulfide $\mathbf{3 g}(16.9 \mathrm{mg}$, $0.1 \mathrm{mmol})$, after chromatography $(n$-hexane/AcOEt $=1 / 1$ to $3 / 7)$ the corresponding $N$-benzoyl sulfimide $\mathbf{4 a g}$ was obtained as white solid (28.8 mg, > 99\%).; Colorless crystals were obtained from $\mathrm{CHCl}_{3} /$ Hexane.; m.p. 109.2-109.3 ${ }^{\circ} \mathrm{C}$.; The enantiomeric excess of the sulfimide 4ag was determined to be $94 \%$ ee by HPLC analysis using a chiral stationary phase [DAICEL CHIRALPAK IF-3 (Hexane $/ i-\mathrm{PrOH}=6 / 4,0.5 \mathrm{~mL} / \mathrm{min}), t_{\mathrm{r}}($ Major $)=37.3 \mathrm{~min}, t_{\mathrm{r}}$ $($ Minor $)=21.9 \mathrm{~min}]$; pale yellow solid; $[\alpha]_{\mathrm{D}}{ }^{25}=-183.3\left(c=1.24, \mathrm{CHCl}_{3}\right) ;{ }^{1} \mathrm{H}$ NMR $(400$ $\left.\mathrm{MHz}, \mathrm{CDCl}_{3}\right) \delta 8.37(\mathrm{~d}, J=8.8 \mathrm{~Hz}, 2 \mathrm{H}), 8.14(\mathrm{~d}, J=8.3 \mathrm{~Hz}, 2 \mathrm{H}), 8.00(\mathrm{~d}, J=8.3 \mathrm{~Hz}, 2 \mathrm{H})$, 7.43 (pseud-dt, $J=29.1,7.8 \mathrm{~Hz}, 3 \mathrm{H}), 2.97$ (s, 3H) ppm; ${ }^{13} \mathrm{C}$ NMR $\left(100 \mathrm{MHz}, \mathrm{CDCl}_{3}\right) \delta 177.1$, 150.0, 143.4, 135.5, 131.3, 128.8, 128.0, 127.7, 124.9, 34.6 ppm; FT-IR (KBr): 3539, 3445, $3065,1626,1587,1558,1506,1450,1395,1248,1206,1150,1099,993,881,853,800,748$ $\mathrm{cm}^{-1}$; HRESI-MS (m/z) $\left[\mathrm{M}+\mathrm{Na}^{+}\right]$, calcd for $\left[\mathrm{C}_{14} \mathrm{H}_{12} \mathrm{~N}_{2} \mathrm{NaO}_{3} \mathrm{~S}\right]^{+} 311.0461$, found: $\mathrm{m} / \mathrm{z}=$ 311.0461 .

\subsection{6. (-)- $N$-Benzoyl 3-bromophenyl methyl sulfimide (4ah)}<smiles>C[S-](C)(=NC(=O)c1cccc(Br)c1)c1ccccc1</smiles>

Following typical experiment 3.1, 3-phenyl-1,4,2-dioxazol-5-one 1a (21.2 $\mathrm{mg}, 0.13 \mathrm{mmol})$ and 3-bromophenyl methyl sulfide $\mathbf{3 h}(13.4 \mu \mathrm{L}, 0.1$ mmol), after chromatography ( $n$-hexane/AcOEt $=1 / 1$ to $3 / 7$ ) the corresponding $N$-benzoyl sulfimide $\mathbf{4 a h}$ was obtained as white solid (32.2 $\mathrm{mg},>99 \%$ ).; The enantiomeric excess of the sulfimide 4ah was determined to be $94 \%$ ee by HPLC analysis using a chiral stationary phase

[DAICEL CHIRALPAK IF-3 (Hexane $/ i-\mathrm{PrOH}=7 / 3,0.5 \mathrm{~mL} / \mathrm{min}), t_{\mathrm{r}}($ Major $)=24.2 \mathrm{~min}, t_{\mathrm{r}}$ $($ Minor $)=20.7 \mathrm{~min}]$; white solid; $[\alpha]_{\mathrm{D}}{ }^{25}=-161.1\left(c=1.62, \mathrm{CHCl}_{3}\right) ;{ }^{1} \mathrm{H}$ NMR $(400 \mathrm{MHz}$, $\left.\mathrm{CDCl}_{3}\right) \delta 8.16(\mathrm{dd}, J=8.5,1.2 \mathrm{~Hz}, 2 \mathrm{H}), 7.97(\mathrm{t}, J=2.0 \mathrm{~Hz}, 1 \mathrm{H}), 7.74(\mathrm{dd}, J=7.8,1.0 \mathrm{~Hz}$, $1 \mathrm{H}), 7.68(\mathrm{~d}, J=7.8 \mathrm{~Hz} .1 \mathrm{H}), 7.48-7.38(\mathrm{~m}, 4 \mathrm{H}), 2.92(\mathrm{~s}, 3 \mathrm{H}) \mathrm{ppm} ;{ }^{13} \mathrm{C}$ NMR $(100 \mathrm{MHz}$, $\left.\mathrm{CDCl}_{3}\right) \delta 176.9,138.2,135.9,135.3,131.3,131.0,129.3,128.7,127.8,125.2,123.9,35.1$ ppm; FT-IR (KBr): 3539, 3445, 3065, 1626, 1587, 1558, 1506, 1450, 1395, 1248, 1206, 1150 , $1099,993,881,853,800,748 \mathrm{~cm}^{-1}$; HRESI-MS $(\mathrm{m} / \mathrm{z})\left[\mathrm{M}+\mathrm{Na}^{+}\right]$, calcd for $\left[\mathrm{C}_{14} \mathrm{H}_{12} \mathrm{BrNNaOS}\right]^{+}$343.9715, found: $\mathrm{m} / \mathrm{z}=343.9720$.

\subsection{7. (-)-N-Benzoyl 2-bromophenyl methyl sulfimide (4ai)}

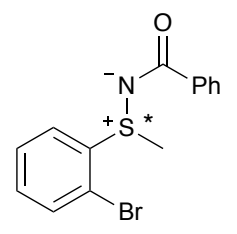

Following typical experiment 3.1, 3-phenyl-1,4,2-dioxazol-5-one 1a (21.2 $\mathrm{mg}, 0.13 \mathrm{mmol})$ and 2-bromophenyl methyl sulfide $3 \mathbf{i}(13.2 \mu \mathrm{L}, 0.1 \mathrm{mmol})$, after chromatography ( $n$-hexane/AcOEt $=1 / 1$ to $3 / 7)$ the corresponding $N$ benzoyl sulfimide 4ai was obtained as white solid (32.2 mg, > 99\%).; The enantiomeric excess of the sulfimide 4ai was determined to be $95 \%$ ee by HPLC analysis using a chiral stationary phase [DAICEL CHIRALPAK IF-3 
$($ Hexane $/ i-\mathrm{PrOH}=6 / 4,0.5 \mathrm{~mL} / \mathrm{min}), t_{\mathrm{r}}($ Major $)=20.3 \mathrm{~min}, t_{\mathrm{r}}$ (Minor) $\left.=18.8 \mathrm{~min}\right]$; white solid; $[\alpha]_{\mathrm{D}}{ }^{25}=-195.5\left(c=1.45, \mathrm{CHCl}_{3}\right) ;{ }^{1} \mathrm{H} \mathrm{NMR}\left(400 \mathrm{MHz}, \mathrm{CDCl}_{3}\right) \delta 8.20(\mathrm{dd}, J=6.8,1.5$ $\mathrm{Hz}, 2 \mathrm{H}), 7.95$ (dd, $J=7.8,1.5 \mathrm{~Hz}, 1 \mathrm{H}), 7.67$ (dd, J = 7.8, $1.5 \mathrm{~Hz}, 1 \mathrm{H}), 7.54-7.39$ (m, 5H), 2.95 (s, 3H) ppm; ${ }^{13} \mathrm{C} \mathrm{NMR}\left(100 \mathrm{MHz}, \mathrm{CDCl}_{3}\right) \delta 176.7,136.2,136.1,133.6,133.1,131.0$, 129.0, 128.8, 127.9, 127.6, 121.7, 33.6 ppm; FT-IR (KBr): 3539, 3445, 3065, 1626, 1587, $1558,1506,1450,1395,1248,1206,1150,1099,993,881,853,800,748 \mathrm{~cm}^{-1}$; HRESI-MS $(\mathrm{m} / \mathrm{z})\left[\mathrm{M}+\mathrm{Na}^{+}\right]$, calcd for $\left[\mathrm{C}_{14} \mathrm{H}_{12} \mathrm{BrNNaOS}\right]^{+} 343.9715$, found: $\mathrm{m} / \mathrm{z}=343.9715$.

\subsection{8. (-)-N-Benzoyl ethyl phenyl sulfimide (4aj)}

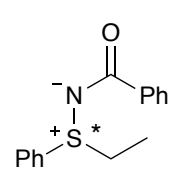

Following typical experiment 3.1, 3-phenyl-1,4,2-dioxazol-5-one 1a (21.2 mg, $0.13 \mathrm{mmol})$ and ethyl phenyl sulfide $3 \mathbf{j}(13.5 \mu \mathrm{L}, 0.1 \mathrm{mmol})$, after chromatography ( $n$-hexane/AcOEt $=1 / 1$ to $3 / 7$ ) the corresponding $N$-benzoyl sulfimide 4aj was obtained as white solid $(15.9 \mathrm{mg}, 62 \%)$.; The enantiomeric excess of the sulfimide 4aj was determined to be $92 \%$ ee by HPLC analysis using a chiral stationary phase [DAICEL CHIRALPAK IF-3 (Hexane $/ i$-PrOH $=6 / 4,0.5$ $\mathrm{mL} / \mathrm{min}), t_{\mathrm{r}}($ Major $)=18.4 \mathrm{~min}, t_{\mathrm{r}}($ Minor $\left.)=18.4 \mathrm{~min}\right]$; white solid; $[\alpha]_{\mathrm{D}}{ }^{25}=-148.9(c=0.78$, $\left.\mathrm{CHCl}_{3}\right) ;{ }^{1} \mathrm{H} \mathrm{NMR}\left(400 \mathrm{MHz}, \mathrm{CDCl}_{3}\right) \delta 8.20(\mathrm{~d}, J=6.8 \mathrm{~Hz}, 2 \mathrm{H}), 7.81(\mathrm{dd}, J=7.6,2.2 \mathrm{~Hz}$, 2H), 7.56-7.50 (m, 3H), 7.41 (pseud-dt, $J=15.0,7.5 \mathrm{~Hz}, 3 \mathrm{H}), 3.22$ (q, $J=7.6 \mathrm{~Hz}, 2 \mathrm{H}), 1.27$ $(\mathrm{t}, J=7.6 \mathrm{~Hz}, 3 \mathrm{H}) \mathrm{ppm} ;{ }^{13} \mathrm{C} \mathrm{NMR}\left(100 \mathrm{MHz}, \mathrm{CDCl}_{3}\right) \delta 176.7,136.6,133.6,132.1,130.7$, 129.7, 128.7, 127.8, 127.3, 43.8, 7.6 ppm; FT-IR (KBr): 3539, 3445, 3065, 1626, 1587, 1558, 1506, 1450, 1395, 1248, 1206, 1150, 1099, 993, 881, 853, 800, $748 \mathrm{~cm}^{-1}$; HRESI-MS (m/z) $\left[\mathrm{M}+\mathrm{Na}^{+}\right]$, calcd for $\left[\mathrm{C}_{15} \mathrm{H}_{15} \mathrm{NNaOS}\right]^{+} 280.0767$, found: $\mathrm{m} / \mathrm{z}=280.0767$.

\subsection{9. (-)- $N$-Benzoyl benzyl methyl sulfimide (4al)}<smiles>CN(C(=O)c1ccccc1)[As](C)Cc1ccccc1</smiles>

Following typical experiment 3.1, 3-phenyl-1,4,2-dioxazol-5-one 1a (21.2 $\mathrm{mg}, 0.13 \mathrm{mmol})$ and benzyl methyl sulfide $\mathbf{3 1}(13.6 \mu \mathrm{L}, 0.1 \mathrm{mmol})$ with (OC)ruthenium-salen $\mathbf{2 b}(4.1 \mathrm{mg}, 4.0 \mu \mathrm{mol}, 4.0 \mathrm{~mol} \%)$ as the catalyst, after chromatography ( $n$-hexane/AcOEt $=1 / 1$ to $3 / 7)$ the corresponding $N$-benzoyl sulfimide 4al was obtained as white solid (25.4 mg, 99\%).; The enantiomeric excess of the sulfimide 4al was determined to be $91 \%$ ee by HPLC analysis using a chiral stationary phase [DAICEL CHIRALPAK AY-3 (Hexane $/ i-\mathrm{PrOH}=6 / 4,0.5 \mathrm{~mL} / \mathrm{min}), t_{\mathrm{r}}$ (Major) $=25.2 \mathrm{~min}, t_{\mathrm{r}}$ $($ Minor $)=18.4 \mathrm{~min}]$; white solid; $[\alpha]_{\mathrm{D}}{ }^{25}=-310.3\left(c=0.32, \mathrm{CHCl}_{3}\right) ;{ }^{1} \mathrm{H}$ NMR $(400 \mathrm{MHz}$, $\left.\mathrm{CDCl}_{3}\right) \delta 8.10(\mathrm{~d}, J=8.8 \mathrm{~Hz}, 2 \mathrm{H}), 7.45-7.34(\mathrm{~m}, 8 \mathrm{H}), 4.61(\mathrm{~d}, J=12.7 \mathrm{~Hz}, 1 \mathrm{H}), 4.13(\mathrm{~d}, J=$ $12.7 \mathrm{~Hz}, 1 \mathrm{H}), 2.56(\mathrm{~s}, 3 \mathrm{H}) \mathrm{ppm} ;{ }^{13} \mathrm{C} \mathrm{NMR}\left(100 \mathrm{MHz}, \mathrm{CDCl}_{3}\right) \delta 177.2,136.4,130.7,130.5$, 129.2 128.6, 128.5, 127.8, 51.7, 27.6 ppm; FT-IR (KBr): 3539, 3445, 3065, 1626, 1587, 1558, 1506, 1450, 1395, 1248, 1206, 1150, 1099, 993, 881, 853, 800, 748 $\mathrm{cm}^{-1}$; HRESI-MS (m/z) $\left[\mathrm{M}+\mathrm{Na}^{+}\right]$, calcd for $\left[\mathrm{C}_{15} \mathrm{H}_{15} \mathrm{NNaOS}\right]^{+} 280.0767$, found: $\mathrm{m} / \mathrm{z}=280.0767$.

\subsection{0. (+)- $N$-Benzoyl methyl octyl sulfimide (4am)}<smiles>CCCCCCCC[Si](C)(C)N(C)C(=O)c1ccccc1</smiles>

Following typical experiment 3.1, 3-phenyl-1,4,2-dioxazol-5-one 1a (21.2 $\mathrm{mg}, 0.13 \mathrm{mmol})$ and methyl octyl sulfide $3 \mathrm{~m}(19.0 \mu \mathrm{L}, 0.1 \mathrm{mmol})$, after chromatography ( $n$-hexane/AcOEt $=1 / 1$ to $3 / 7)$ the corresponding $N$ benzoyl sulfimide 4am was obtained as white solid (6.7 $\mathrm{mg}, 24 \%)$; The enantiomeric excess of the sulfimide 4am was determined to be $88 \%$ ee by HPLC analysis using a chiral stationary phase DAICEL CHIRALPAK AY-3 (Hexane $/ i$-PrOH $=6 / 4,0.5$ $\mathrm{mL} / \mathrm{min}), t_{\mathrm{r}}($ Major $)=15.7 \mathrm{~min}, t_{\mathrm{r}}($ Minor $\left.)=13.0 \mathrm{~min}\right]$; white solid; $[\alpha]_{\mathrm{D}}{ }^{25}=+90.2(c=0.33$, 
$\mathrm{CHCl} 3) ;{ }^{1} \mathrm{H}$ NMR $\left(400 \mathrm{MHz}, \mathrm{CDCl}_{3}\right) \delta 8.09(\mathrm{~d}, J=7.3 \mathrm{~Hz}, 2 \mathrm{H}), 7.44-7.34(\mathrm{~m}, 3 \mathrm{H}), 3.18(\mathrm{dt}$, $J=12.7,7.8 \mathrm{~Hz}, 1 \mathrm{H}), 2.93(\mathrm{dt}, J=12.7,7.8 \mathrm{~Hz}, 1 \mathrm{H}), 2.73(\mathrm{~s}, 3 \mathrm{H}), 1.84-1.77(\mathrm{~m}, 2 \mathrm{H}), 1.53-$ $1.44(\mathrm{~m}, 2 \mathrm{H}), 1.35-1.20(\mathrm{~m}, 8 \mathrm{H}), 0.88(\mathrm{t}, J=7.3 \mathrm{~Hz}, 3 \mathrm{H}) \mathrm{ppm} ;{ }^{13} \mathrm{C}$ NMR $\left(100 \mathrm{MHz}, \mathrm{CDCl}_{3}\right)$ $\delta 177.1,136.6,130.6,128.5,127.8,46.7,31.7,30.0,29.0,29.0,28.6,23.2,22.6,14.0$ ppm; FT-IR (KBr): 3539, 3445, 3065, 1626, 1587, 1558, 1506, 1450, 1395, 1248, 1206, 1150, 1099 , 993, 881, 853, 800,748 $\mathrm{cm}^{-1}$; HRESI-MS $(\mathrm{m} / \mathrm{z})\left[\mathrm{M}+\mathrm{Na}^{+}\right]$, calcd for $\left[\mathrm{C}_{16} \mathrm{H}_{25} \mathrm{NNaOS}\right]^{+}$ 302.1549 , found: $\mathrm{m} / \mathrm{z}=302.1549$.

\subsection{1. (+)- $N$-Benzoyl methyl 2-propyl sulfimide (4an)}

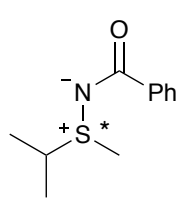

Following typical experiment 3.1, 3-phenyl-1,4,2-dioxazol-5-one 1a $(21.2 \mathrm{mg}$, $0.13 \mathrm{mmol})$ and isopropyl methyl sulfide $3 \mathbf{n}(10.9 \mu \mathrm{L}, 0.1 \mathrm{mmol})$, after chromatography $(n$-hexane/AcOEt $=1 / 1$ to $3 / 7)$ the corresponding $N$-benzoyl sulfimide 4an was obtained as white solid (18.5 mg, 89\%).; The enantiomeric excess of the sulfimide 4an was determined to be $97 \%$ ee by HPLC analysis using a chiral stationary phase [DAICEL CHIRALPAK AY-3 (Hexane $/ i$-PrOH $=6 / 4,0.5$ $\mathrm{mL} / \mathrm{min}), t_{\mathrm{r}}($ Major $)=20.9 \mathrm{~min}, t_{\mathrm{r}}($ Minor $\left.)=18.2 \mathrm{~min}\right]$; white solid; $[\alpha]_{\mathrm{D}}{ }^{25}=+75.9(c=0.88$, $\left.\mathrm{CHCl}_{3}\right) ;{ }^{1} \mathrm{H}$ NMR $\left(400 \mathrm{MHz}, \mathrm{CDCl}_{3}\right) \delta 8.10(\mathrm{dd}, J=8.3,1.5 \mathrm{~Hz}, 2 \mathrm{H}), 7.44-7.34(\mathrm{~m}, 3 \mathrm{H})$, $3.45(\mathrm{qq}, J=6.8,6.8 \mathrm{~Hz}, 1 \mathrm{H}), 2.64(\mathrm{~s}, 3 \mathrm{H}), 1.45(\mathrm{~d}, J=6.8 \mathrm{~Hz}, 3 \mathrm{H}), 1.42(\mathrm{~d}, J=6.8 \mathrm{~Hz}, 3 \mathrm{H})$ ppm; ${ }^{13} \mathrm{C}$ NMR $\left(100 \mathrm{MHz}, \mathrm{CDCl}_{3}\right) \delta 177.0,136.8,130.5,128.5,127.7,47.0,25.8,16.7,15.8$ ppm; FT-IR (KBr): 3539, 3445, 3065, 1626, 1587, 1558, 1506, 1450, 1395, 1248, 1206, 1150 , $1099,993,881,853,800,748 \mathrm{~cm}^{-1}$; HRESI-MS $(\mathrm{m} / \mathrm{z})\left[\mathrm{M}+\mathrm{Na}^{+}\right]$, calcd for $\left[\mathrm{C}_{11} \mathrm{H}_{15} \mathrm{NNaOS}\right]^{+}$ 232.0767, found: $\mathrm{m} / \mathrm{z}=232.0767$.

\subsection{2. (+)- $N$-Benzoyl tert-butyl methyl sulfimide (4ao)}

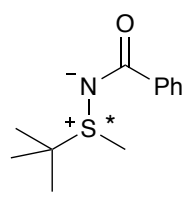

Following typical experiment 3.1, 3-phenyl-1,4,2-dioxazol-5-one 1a (21.2 mg, $0.13 \mathrm{mmol})$ and tert-butyl methyl sulfide 3o $(12.6 \mu \mathrm{L}, 0.1 \mathrm{mmol})$, after chromatography ( $n$-hexane/AcOEt $=1 / 1$ to $3 / 7$ ) the corresponding $N$-benzoyl sulfimide 4 ao was obtained as white solid (16.8 mg, 75\%).; The enantiomeric excess of the sulfimide 4 ao was determined to be $98 \%$ ee by HPLC analysis using a chiral stationary phase [DAICEL CHIRALPAK AY-3 (Hexane/ $i$-PrOH $=6 / 4,0.5$ $\mathrm{mL} / \mathrm{min}), t_{\mathrm{r}}($ Major $)=20.5 \mathrm{~min}, t_{\mathrm{r}}($ Minor $\left.)=17.6 \mathrm{~min}\right]$; white solid; $[\alpha]_{\mathrm{D}}{ }^{25}=+204.6(c=1.43$, $\left.\mathrm{CHCl}_{3}\right) ;{ }^{1} \mathrm{H} \mathrm{NMR}\left(400 \mathrm{MHz}, \mathrm{CDCl}_{3}\right) \delta 8.13(\mathrm{~d}, J=6.8 \mathrm{~Hz}, 2 \mathrm{H}), 7.43-7.34(\mathrm{~m}, 3 \mathrm{H}), 2.57(\mathrm{~s}$, $3 \mathrm{H}), 1.45(\mathrm{~s}, 9 \mathrm{H}) \mathrm{ppm} ;{ }^{13} \mathrm{C} \mathrm{NMR}\left(100 \mathrm{MHz}, \mathrm{CDCl}_{3}\right) \delta 177.0,137.3,130.4,128.6,127.6,52.3$, 24.7, 24.1 ppm; FT-IR (KBr): 3539, 3445, 3065, 1626, 1587, 1558, 1506, 1450, 1395, 1248, $1206,1150,1099,993,881,853,800,748 \mathrm{~cm}^{-1}$; HRESI-MS $(\mathrm{m} / \mathrm{z})\left[\mathrm{M}+\mathrm{Na}^{+}\right]$, calcd for $\left[\mathrm{C}_{12} \mathrm{H}_{17} \mathrm{NNaOS}\right]^{+} 246.0923$, found: $\mathrm{m} / \mathrm{z}=246.0923$.

\subsection{3. (-)- $N$-Benzoyl ethyl propyl sulfimide (4ap)}

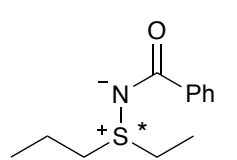

Following typical experiment 3.1, 3-phenyl-1,4,2-dioxazol-5-one 1a (21.2 $\mathrm{mg}, 0.13 \mathrm{mmol})$ and ethyl propyl sulfide $\mathbf{3 p}(12.5 \mu \mathrm{L}, 0.1 \mathrm{mmol})$, after chromatography $(n$-hexane/AcOEt $=1 / 1$ to $3 / 7)$ the corresponding $N$-benzoyl sulfimide 4ap was obtained as white solid $(8.9 \mathrm{mg}, 40 \%)$.; The enantiomeric excess of the sulfimide 4ap was determined to be $75 \%$ ee by HPLC analysis using a chiral stationary phase [DAICEL CHIRALPAK AY-3 (Hexane $/ i$-PrOH $=6 / 4,0.5 \mathrm{~mL} / \mathrm{min}), t_{\mathrm{r}}$ $($ Major $)=18.3 \mathrm{~min}, t_{\mathrm{r}}($ Minor $\left.)=16.8 \mathrm{~min}\right]$; white solid; $[\alpha]_{\mathrm{D}}{ }^{25}=-48.1\left(c=0.82, \mathrm{CHCl}_{3}\right) ;{ }^{1} \mathrm{H}$ NMR $\left(400 \mathrm{MHz}, \mathrm{CDCl}_{3}\right) \delta 8.10(\mathrm{~d}, J=7.3 \mathrm{~Hz}, 2 \mathrm{H}), 7.44-7.34(\mathrm{~m}, 3 \mathrm{H}), 3.12-3.03(\mathrm{~m}, 3 \mathrm{H})$, 
$2.88(\mathrm{dt}, J=7.6,12.7 \mathrm{~Hz}, 1 \mathrm{H}), 1.85(\mathrm{tq}, J=7.3,15.1 \mathrm{~Hz}, 2 \mathrm{H}), 1.40(\mathrm{t}, J=7.6 \mathrm{~Hz}, 3 \mathrm{H}), 1.12$ $(\mathrm{t}, J=7.3 \mathrm{~Hz}, 3 \mathrm{H}) \mathrm{ppm} ;{ }^{13} \mathrm{C}$ NMR $\left(100 \mathrm{MHz}, \mathrm{CDCl}_{3}\right) \delta 177.4,136.8,130.5,128.6,127.7$, 46.1, 38.7, 17.1, 13.3, 7.6 ppm; FT-IR (KBr): 3539, 3445, 3065, 1626, 1587, 1558, 1506, $1450,1395,1248,1206,1150,1099,993,881,853,800,748 \mathrm{~cm}^{-1}$; HRESI-MS (m/z) $[\mathrm{M}+$ $\mathrm{Na}^{+}$, calcd for $\left[\mathrm{C}_{12} \mathrm{H}_{17} \mathrm{NNaOS}\right]^{+} 246.0923$, found: $\mathrm{m} / \mathrm{z}=246.0923$.

\subsection{4. (+)- $N$-Benzoyl methyl phenyl sulfoximine (5aa)}<smiles>CS(C)(=O)(c1ccccc1)N(C(=O)c1ccccc1)c1ccccc1</smiles>

Following typical experiment 3.3, $(+)-N$-Benzoyl methyl phenyl sulfimide 4aa $(24.3 \mathrm{mg}, 0.1 \mathrm{mmol})$, after chromatography $(n$-hexane/AcOEt $=3 / 7)$ the corresponding $N$-Acyl sulfoximine 5aa was obtained as white solid $(22.3 \mathrm{mg}$, $86 \%$ ).; The enantiomeric excess of the sulfoximine 5 aa was determined to be $97 \%$ ee by HPLC analysis using a chiral stationary phase [DAICEL CHIRALPAK IF-3 (Hexane $/ i-\mathrm{PrOH}=6 / 4,0.5 \mathrm{~mL} / \mathrm{min}), t_{\mathrm{r}}($ Major $)=21.0 \mathrm{~min}, t_{\mathrm{r}}($ Minor $)=$ $18.3 \mathrm{~min}]$; white solid; $\left.[\alpha]_{\mathrm{D}}{ }^{25}=+3.6\left(c=1.04, \mathrm{CHCl}_{3}\right) ;{ }^{1} \mathrm{H} \mathrm{NMR}\left(400 \mathrm{MHz}, \mathrm{CDCl}_{3}\right)\right) \delta 8.17$ $(\mathrm{d}, J=7.3 \mathrm{~Hz}, 2 \mathrm{H}), 8.06(\mathrm{~d}, J=7.3 \mathrm{~Hz}, 2 \mathrm{H}), 7.69(\mathrm{t}, J=7.3 \mathrm{~Hz}, 1 \mathrm{H}), 7.62$ (pseud-t, $J=7.6$ $\mathrm{Hz}, 2 \mathrm{H}$ ), 7.51 (pseud-t, $J=7.3 \mathrm{~Hz}, 1 \mathrm{H}$ ), 7.41 (pseud-t, $J=7.3 \mathrm{~Hz}, 2 \mathrm{H}$ ), 3.47 (s, 3H) ppm; ${ }^{13} \mathrm{C}$ $\operatorname{NMR}\left(100 \mathrm{MHz}, \mathrm{CDCl}_{3}\right) \delta 174.2,139.1,135.6,133.8,132.1,129.6,129.4,128.0,127.2,44.4$ ppm; FT-IR (KBr): 3065, 3019, 2926, 1618, 1566, 1447, 1396, 1281, 1217, 1134, 1074, 974, 922, 833, 716, $671 \mathrm{~cm}^{-1}$; HRESI-MS $(\mathrm{m} / \mathrm{z})\left[\mathrm{M}+\mathrm{Na}^{+}\right]$, calcd for $\left[\mathrm{C}_{14} \mathrm{H}_{13} \mathrm{NNaO}_{2} \mathrm{~S}\right]^{+} 282.0559$, found: $\mathrm{m} / \mathrm{z}=282.0559$.

\subsection{5. (R)-(-)- $N$-Acyl methyl phenyl sulfoximine (5pa)}<smiles>CC(=O)N=S(C)(=O)c1ccccc1</smiles>

Following typical experiment 3.3, $(R)-(-)-N$-Acetyl methyl phenyl sulfimide 4pa $(12.6 \mathrm{mg}, 69.5 \mu \mathrm{mol})$, after chromatography $(n$-hexane/AcOEt $=3 / 7)$ the corresponding $N$-acyl sulfoximine 5pa was obtained as white solid $(5.5 \mathrm{mg}$, $40 \%$ ).; The enantiomeric excess of the sulfoximine 5pa was determined to be $95 \%$ ee by HPLC analysis using a chiral stationary phase [DAICEL CHIRALPAK OZ-3 (Hexane $/ i-\mathrm{PrOH}=1 / 1,0.5 \mathrm{~mL} / \mathrm{min}), t_{\mathrm{r}}$ (Major) $=19.9 \mathrm{~min}, t_{\mathrm{r}}$ (Minor) $\left.=17.8 \mathrm{~min}\right]$; white solid; $[\alpha]_{\mathrm{D}}{ }^{23}=-13.9\left(c=0.55, \mathrm{CHCl}_{3}\right) ;{ }^{\text {ref. }}{ }^{4}[\alpha]_{\mathrm{D}}{ }^{23}=+11.1\left(c=1.0, \mathrm{CHCl}_{3}\right),>99 \%$ ee, $S$ isomer; ${ }^{1} \mathrm{H}$ NMR $\left(400 \mathrm{MHz}, \mathrm{CDCl}_{3}\right) \delta 7.98(\mathrm{dt}, J=0.8,7.2 \mathrm{~Hz}, 2 \mathrm{H}), 7.68(\mathrm{tt}, J=0.8,7.2 \mathrm{~Hz}$, $1 \mathrm{H}), 7.60$ (ddt, $J=0.8,7.2,7.2 \mathrm{~Hz}, 2 \mathrm{H}), 3.34(\mathrm{~s}, 3 \mathrm{H}), 2.16(\mathrm{~s}, 3 \mathrm{H}) \mathrm{ppm} ;{ }^{13} \mathrm{C}$ NMR $(100 \mathrm{MHz}$, $\left.\mathrm{CDCl}_{3}\right) \delta 180.2,138.8,133.7,129.6,127.1,44.1,26.8$ ppm; FT-IR (KBr): 2980, 2918, 2847, 1634, 1360, 1263, 1211, 1094, 1032, 966, 827, 743, 685, $617 \mathrm{~cm}^{-1}$; HRESI-MS (m/z) [M + $\left.\mathrm{Na}^{+}\right]$, calcd for $\left[\mathrm{C}_{9} \mathrm{H}_{11} \mathrm{NNaO}_{2} \mathrm{~S}\right]^{+} 220.0403$, found: $\mathrm{m} / \mathrm{z}=220.0403$. 
5. ${ }^{1} \mathrm{H}$ and ${ }^{13} \mathrm{C}$ NMR spectra data.

5.1. (-)- $N$-Benzoyl methyl phenyl sulfimide (4aa)
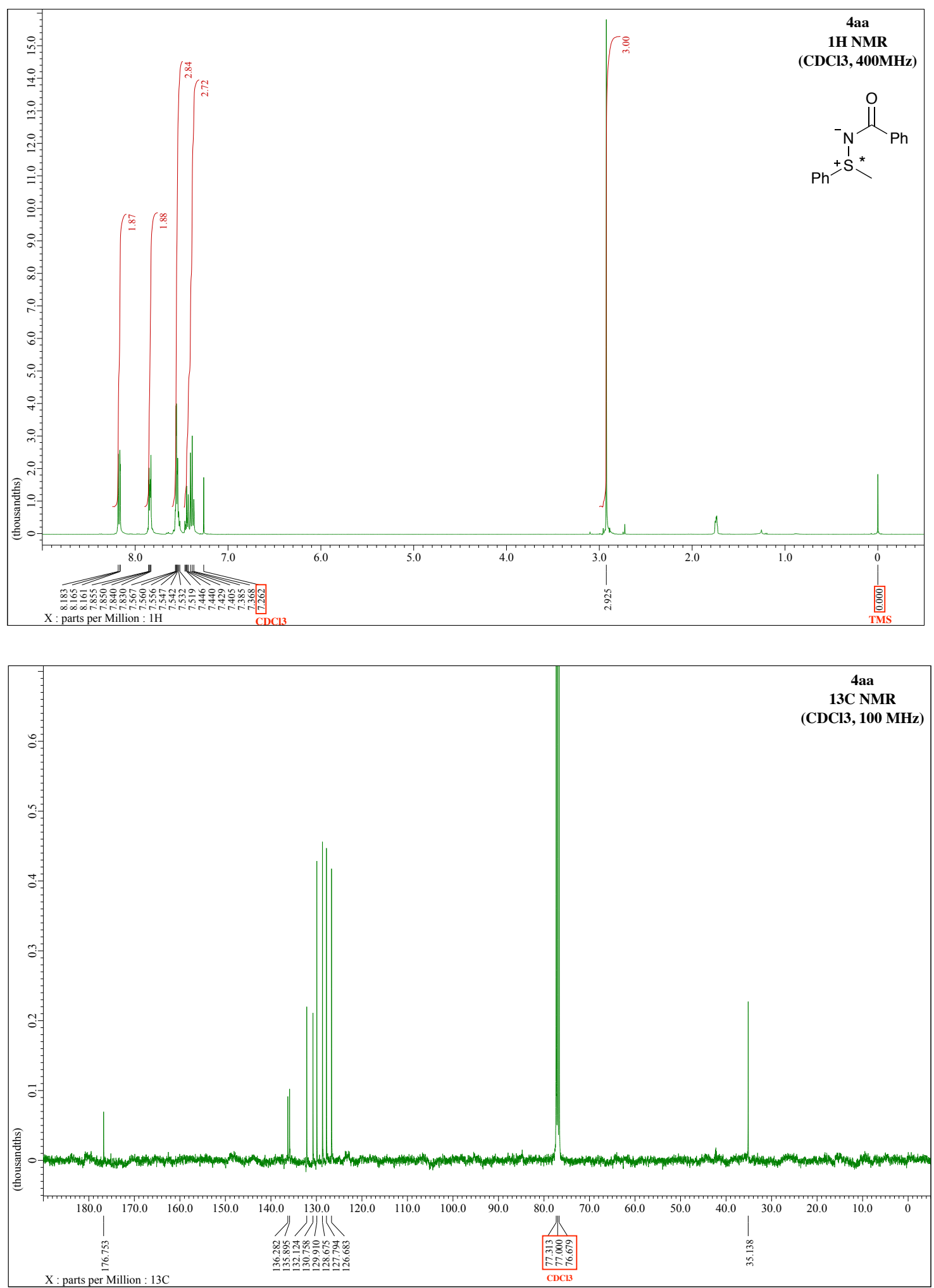
5.2. (-)- $N$-(4-Methylbenzoyl) methyl phenyl sulfimide (4ba)
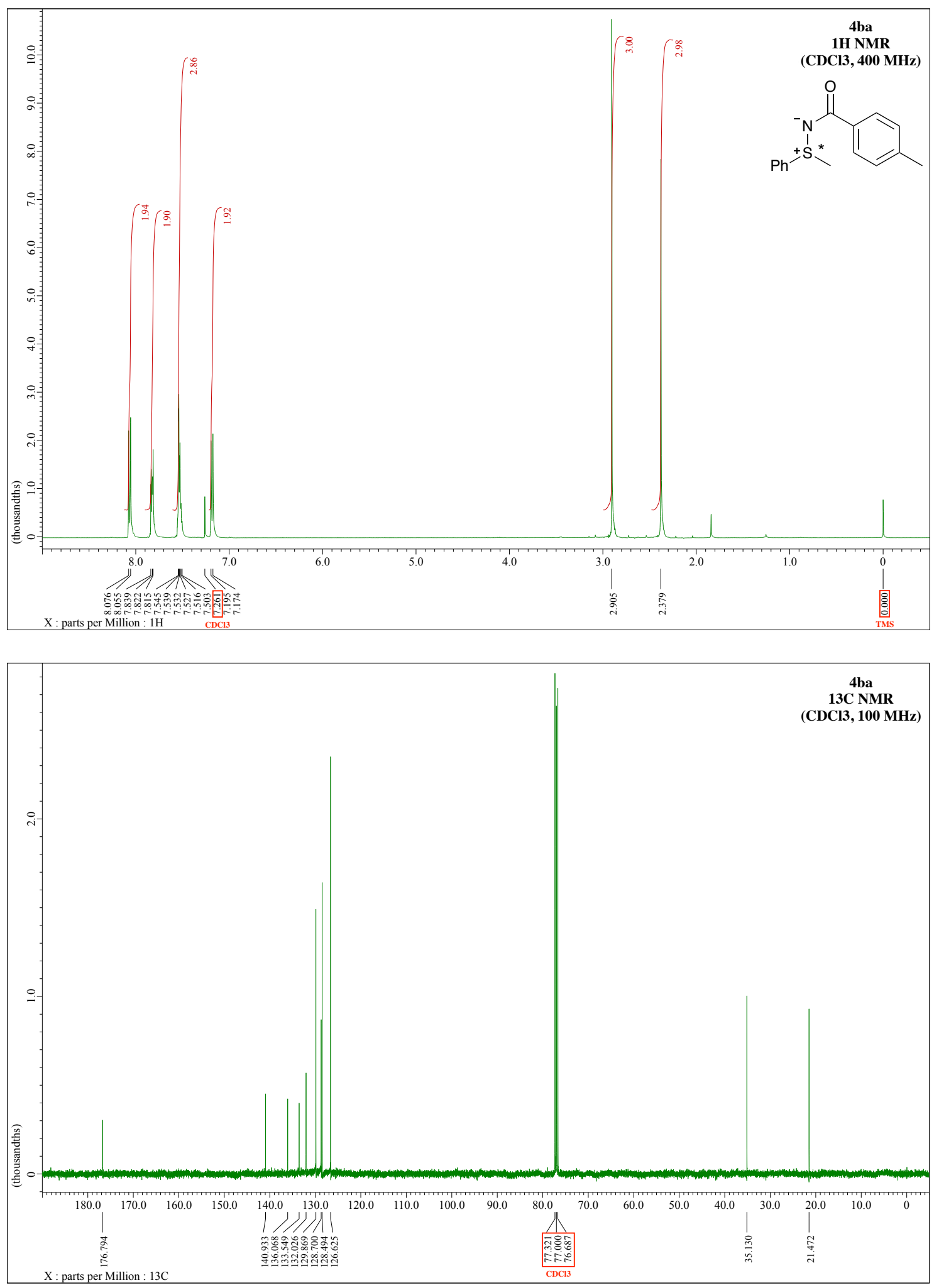
5.3. (-)-N-(4-Methoxylbenzoyl) methyl phenyl sulfimide (4ca)
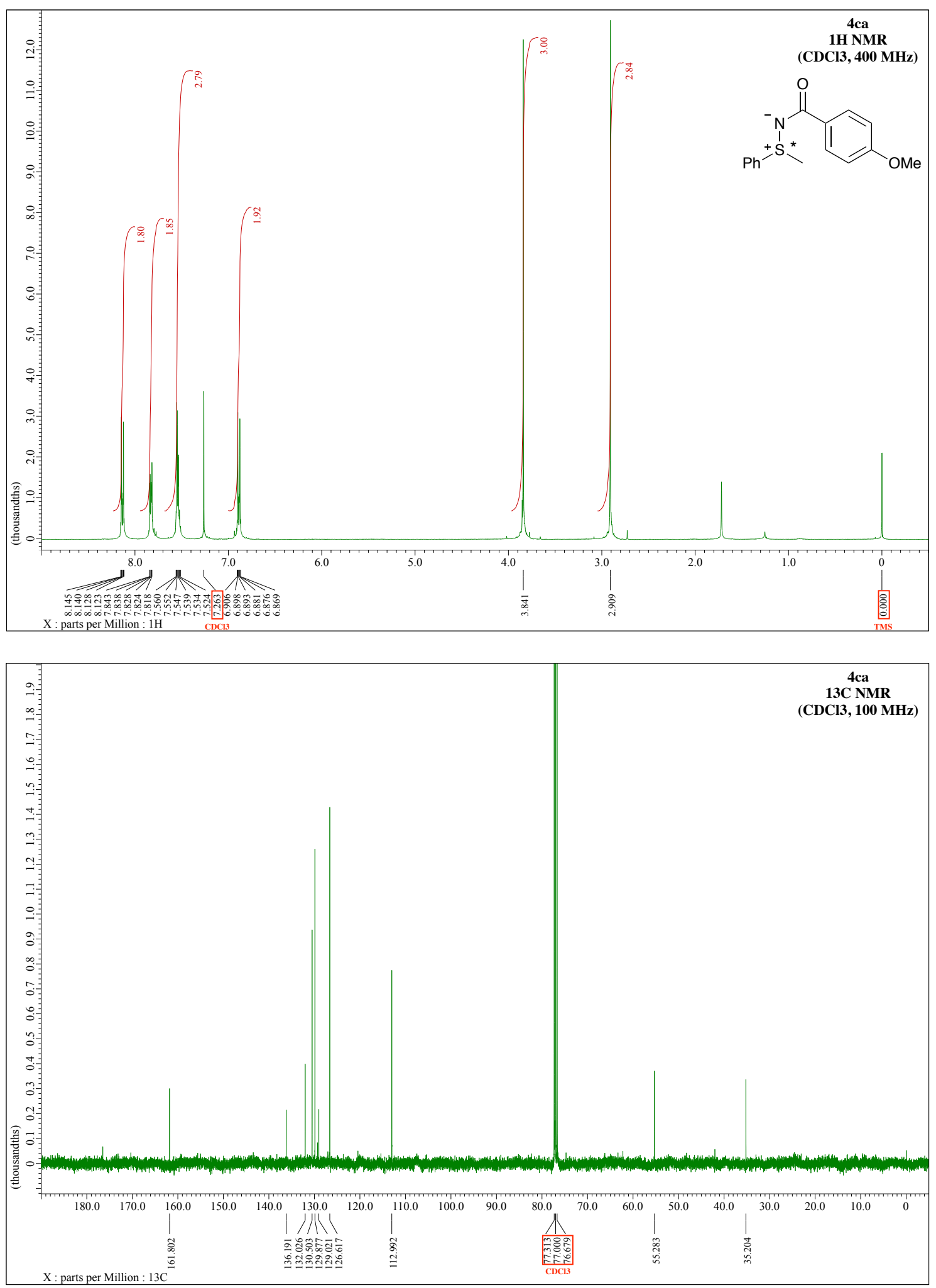
5.4. (-)- $N$-(4-Bromobenzoyl) methyl phenyl sulfimide (4da)
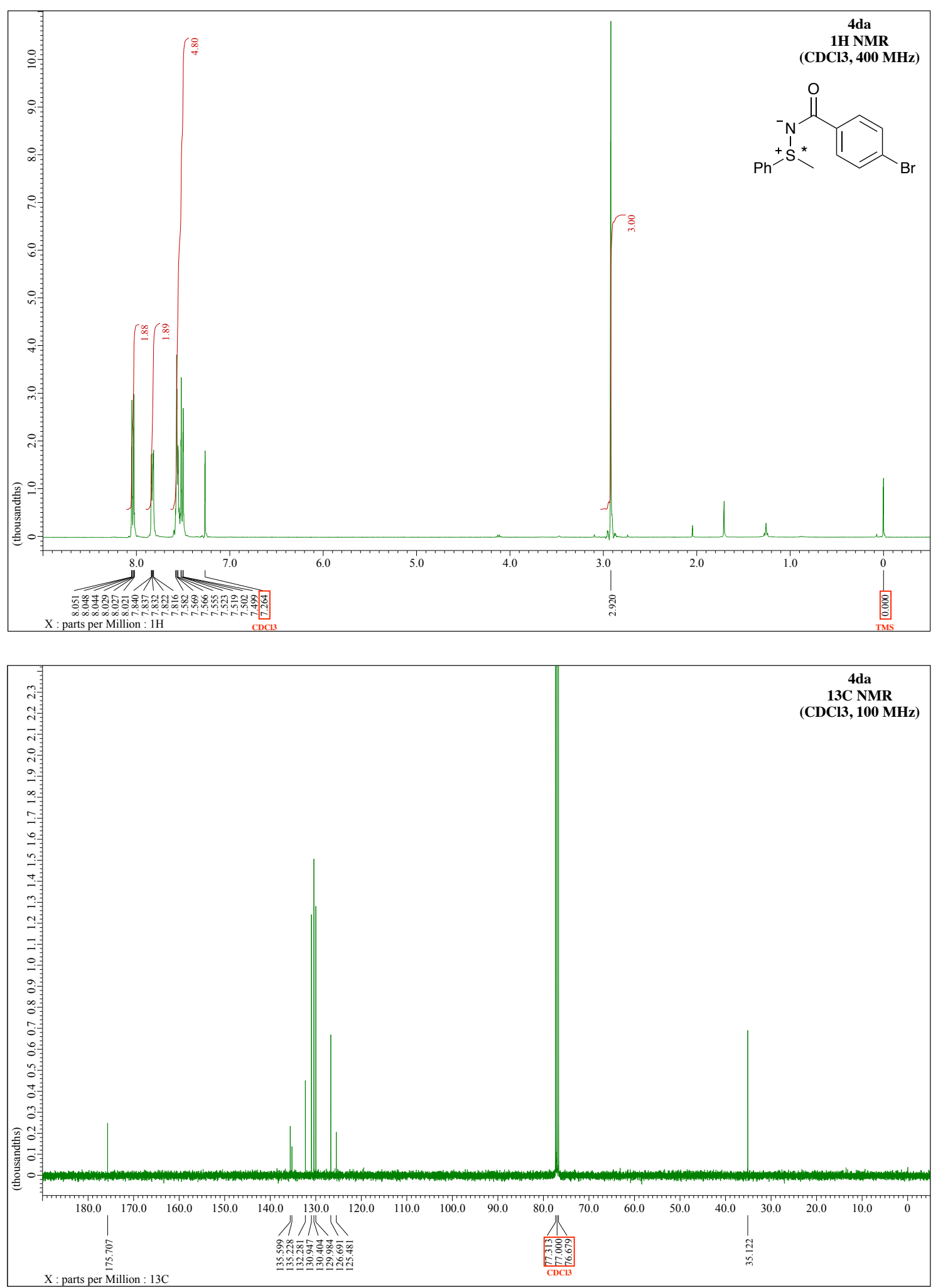
5.5. (-)- $N$-(4-Chlorobenzoyl) methyl phenyl sulfimide (4ea)
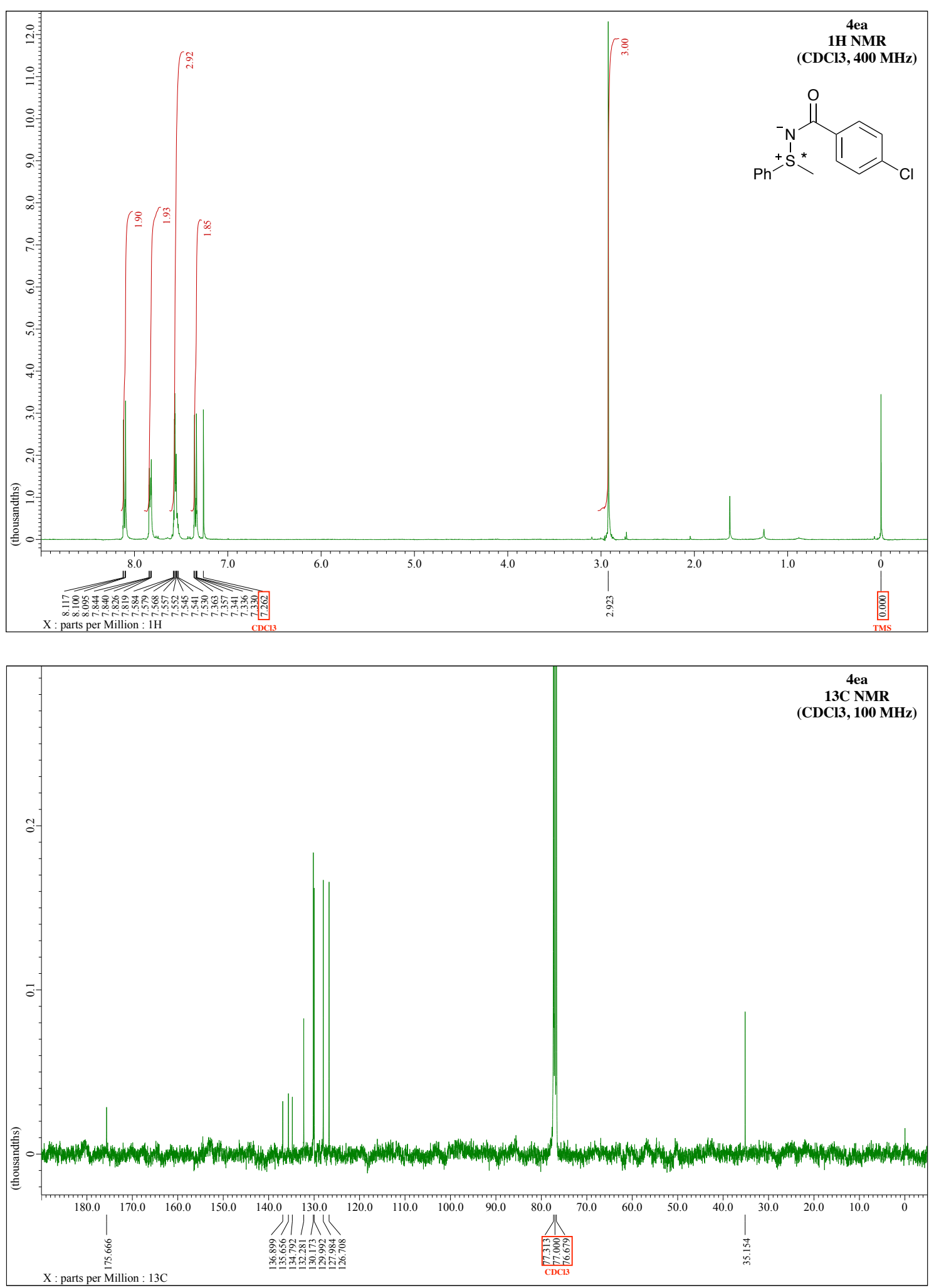
5.6. (-)- $N$-(4-Fluorobenzoyl) methyl phenyl sulfimide (4fa)
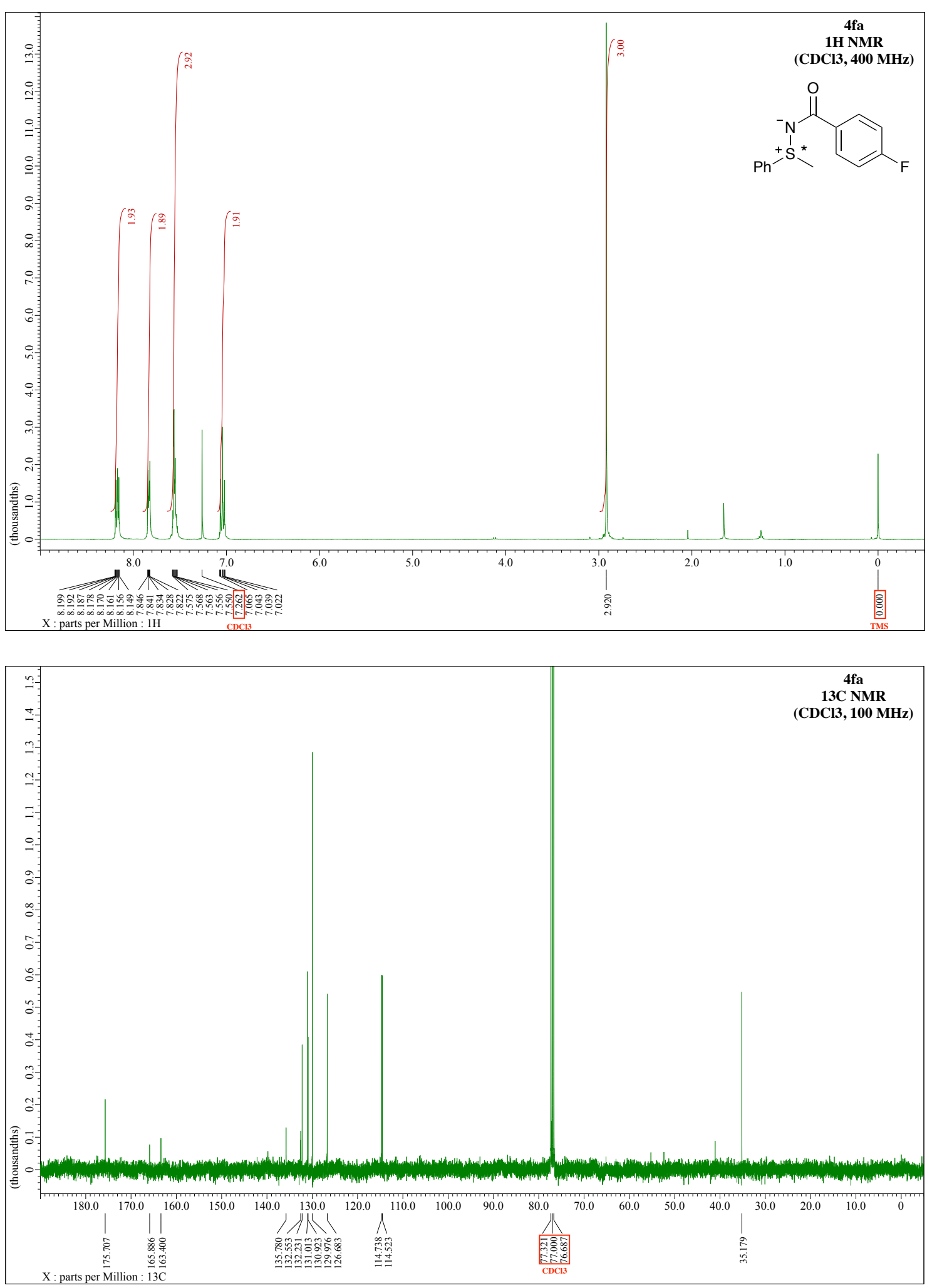
5.7. (-)- $N$-(4-Trifluoromethylbenzoyl) methyl phenyl sulfimide (4ga)
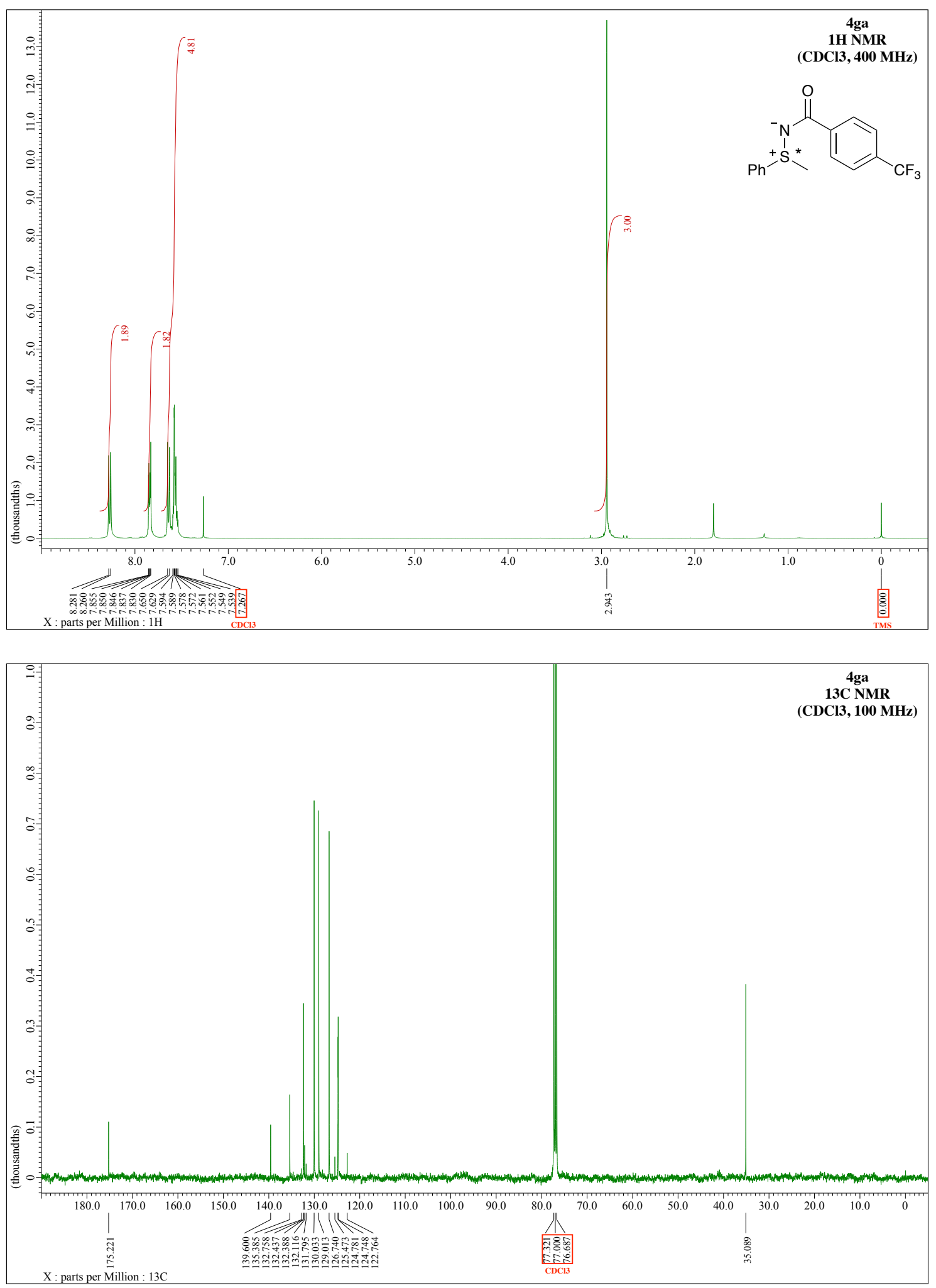
5.8. (-)- $N$-(3-Methylbenzoyl) methyl phenyl sulfimide (4ha)
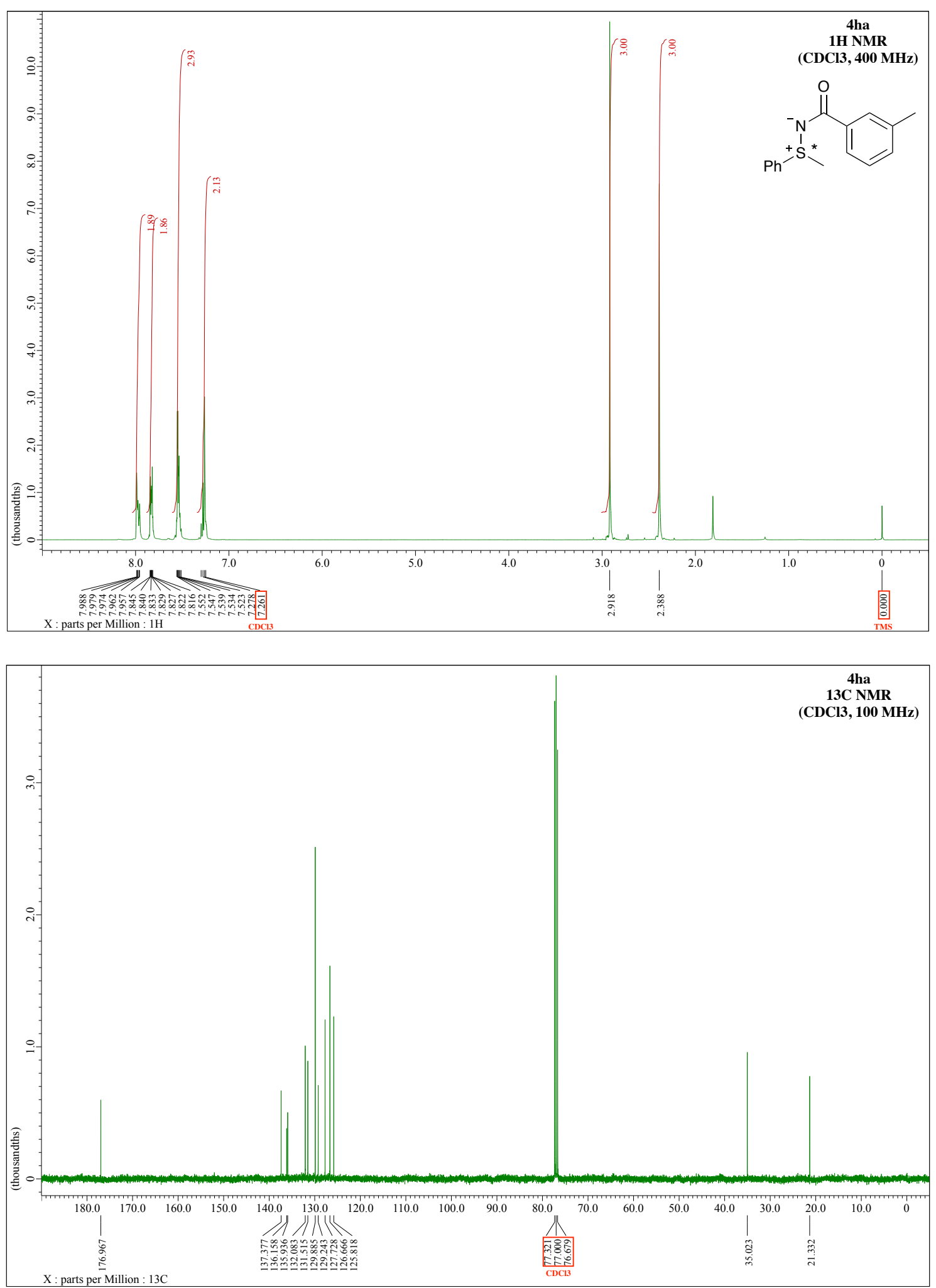
5.9. (-)- $N-(3-$ Bromobenzoyl) methyl phenyl sulfimide (4ia)
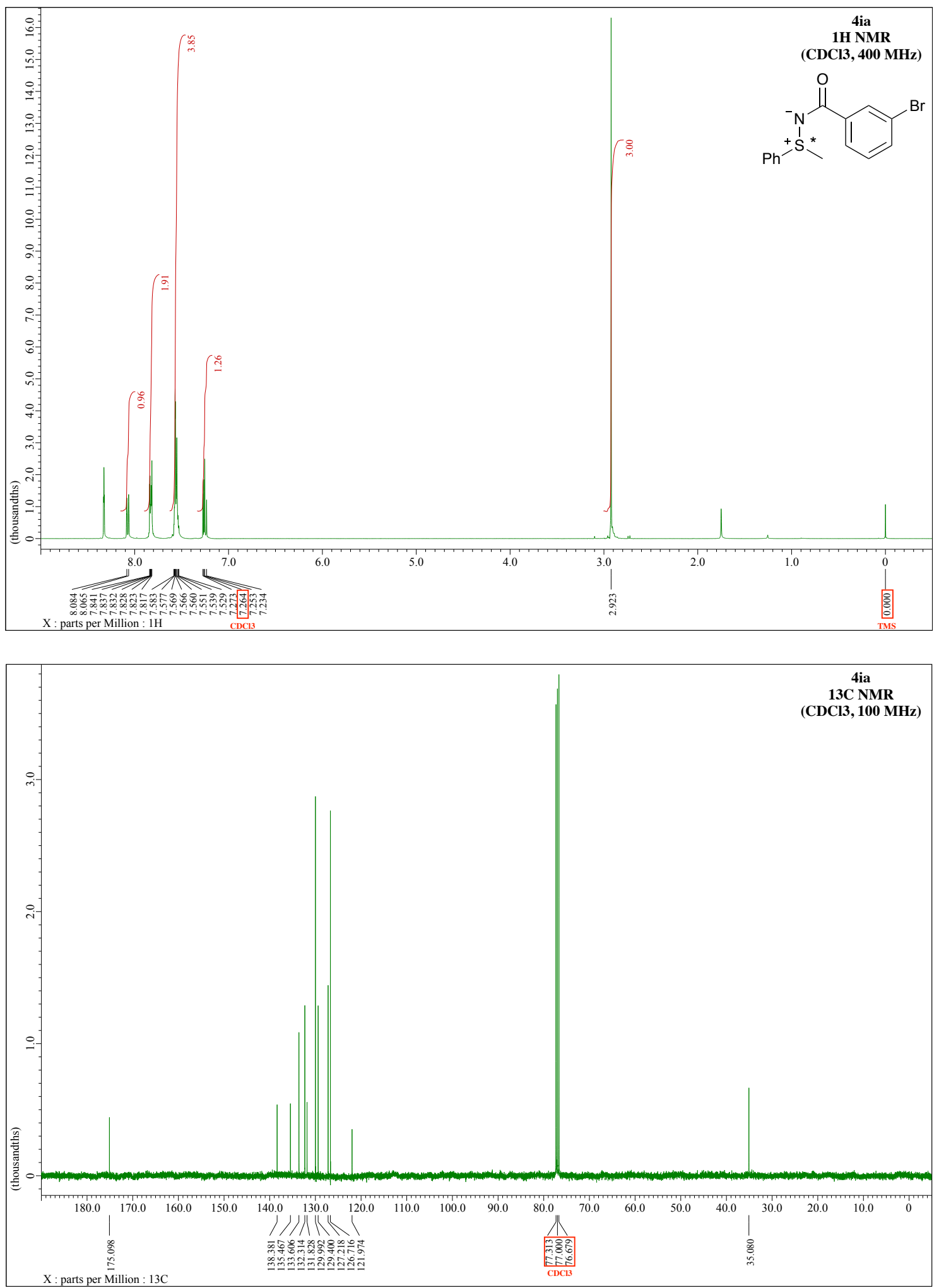
6.10. (-)- $N$-(3-Chlorobenzoyl) methyl phenyl sulfimide (4ja)
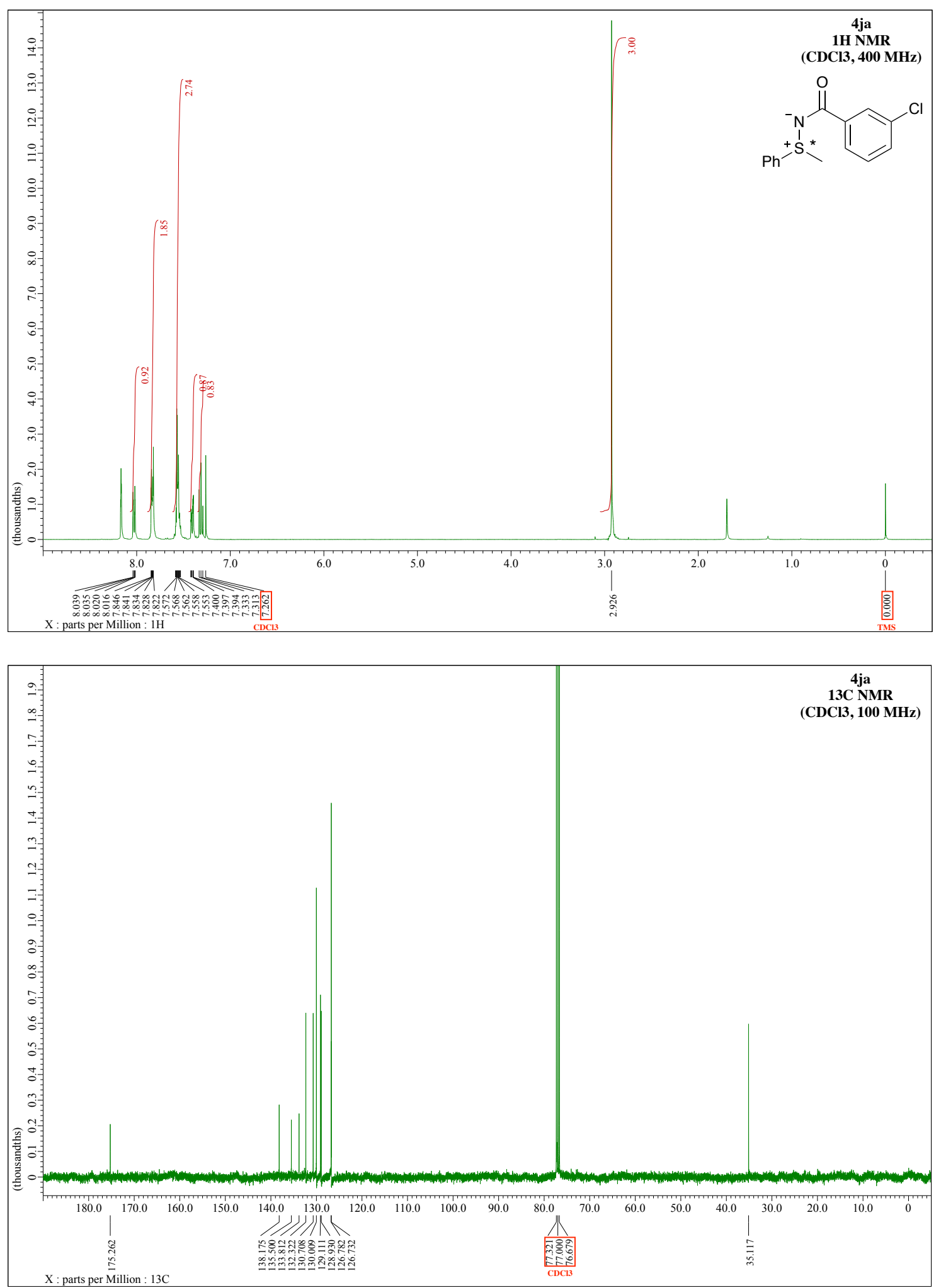
5.11. (-)- $N$-(3-Fluorobenzoyl) methyl phenyl sulfimide (4ka)
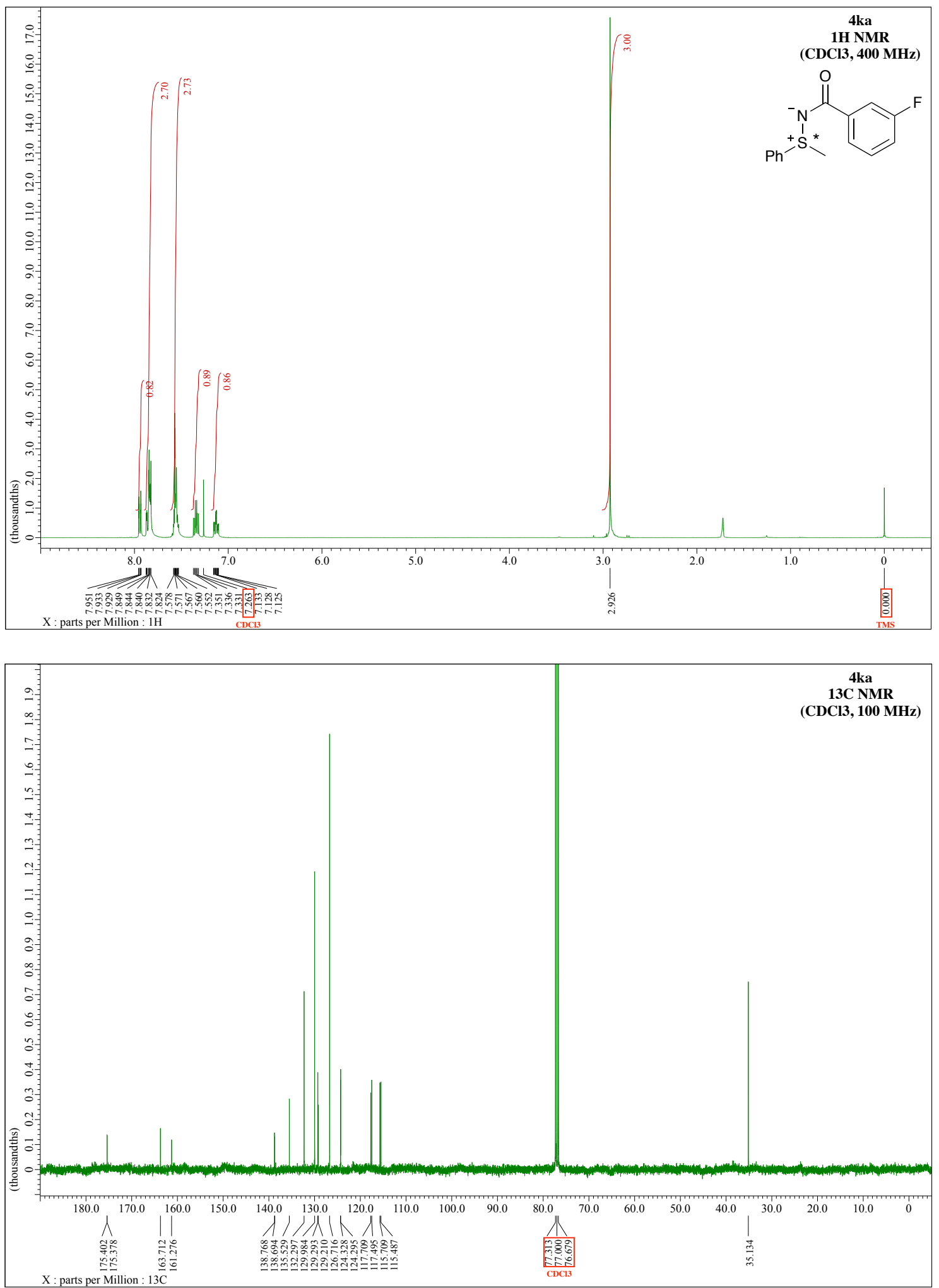
5.12. (-)-N-(2-Methylbenzoyl) methyl phenyl sulfimide (4la)
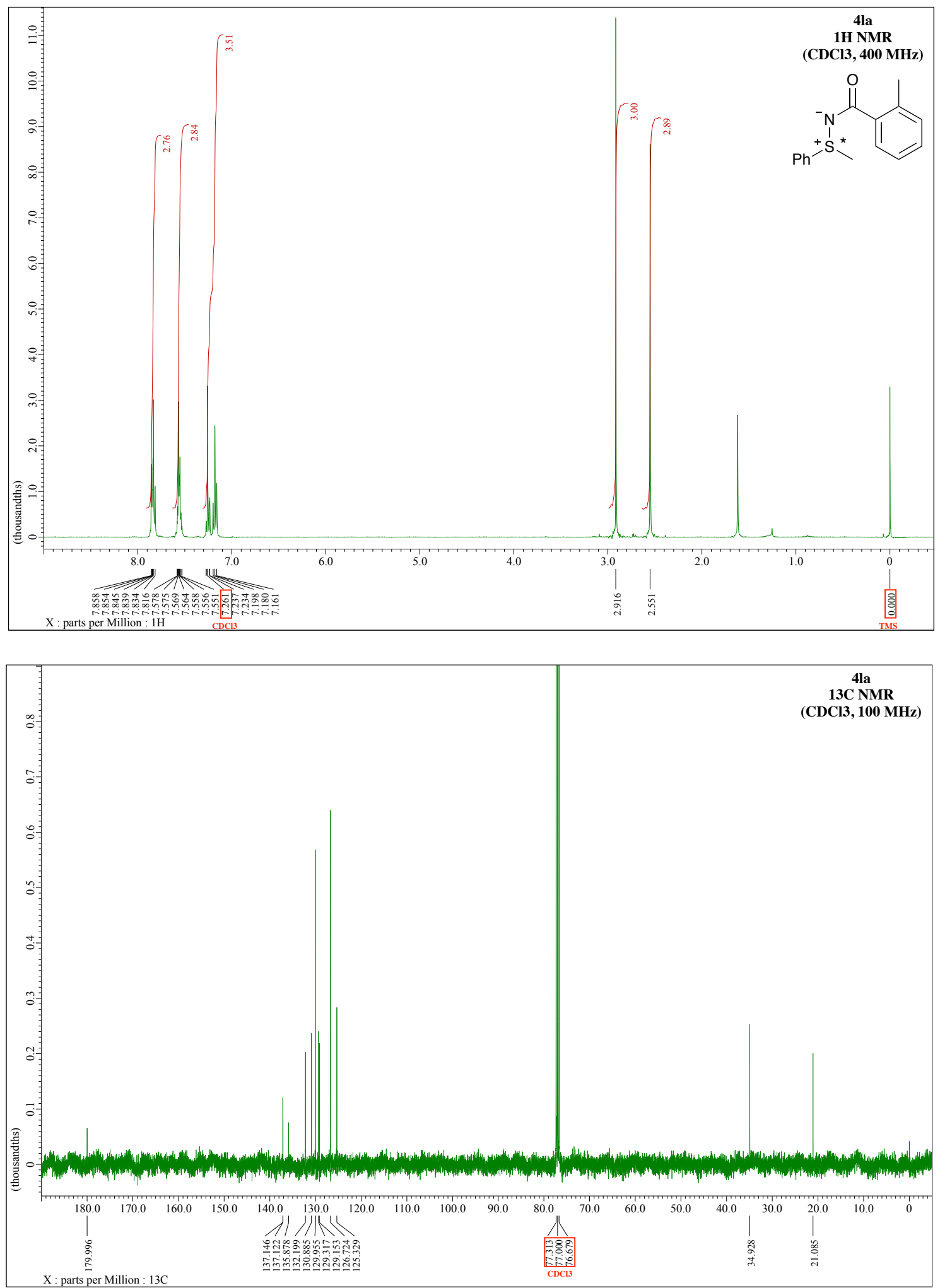
5.13. (-)- $N$-(2-Bromobenzoyl) methyl phenyl sulfimide (4ma)
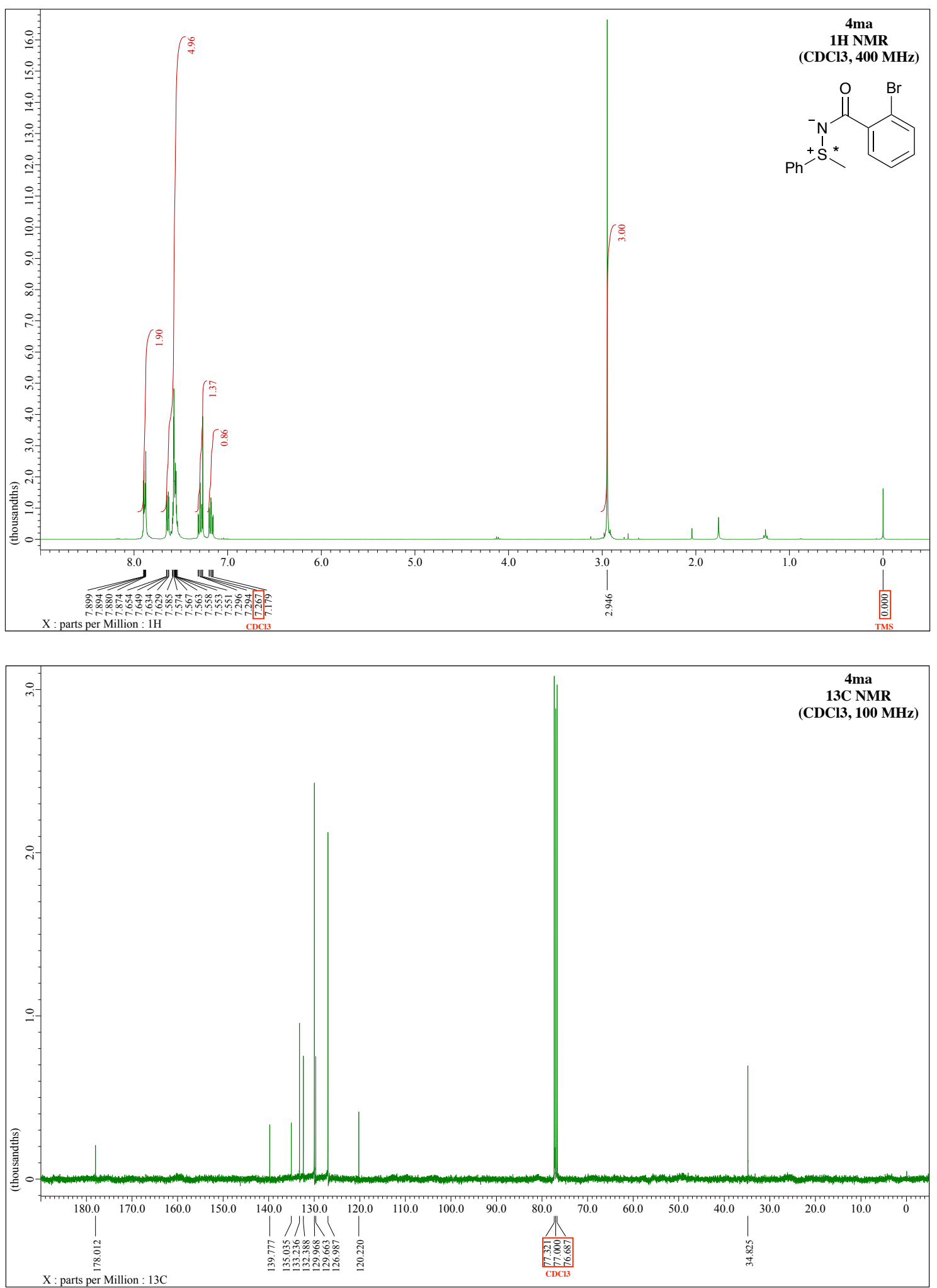
5.14. (-)- $N$-(2-Chlorobenzoyl) methyl phenyl sulfimide (4na)
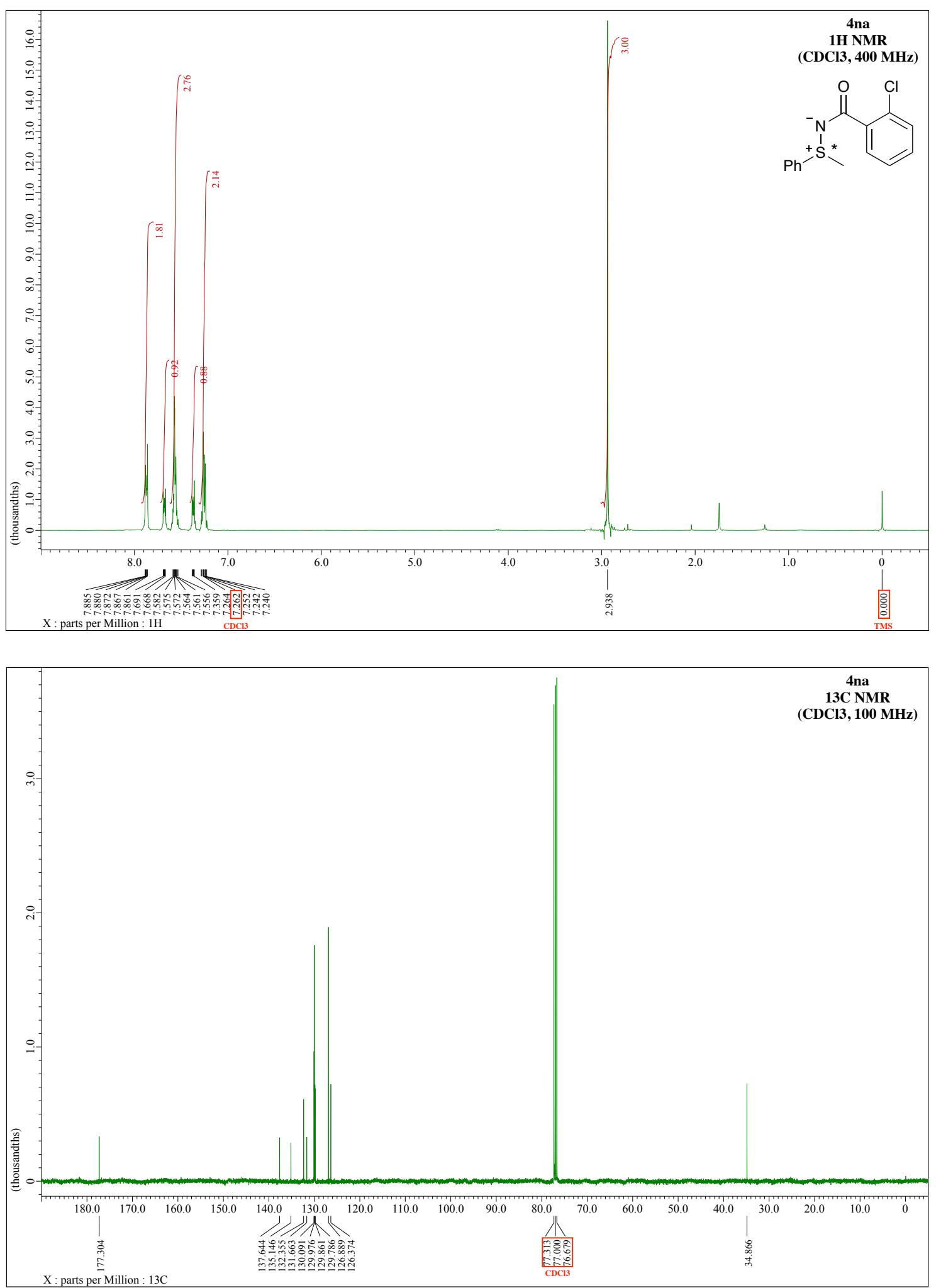
5.15. (-)- $N$-(2-Fluorobenzoyl) methyl phenyl sulfimide (40a)
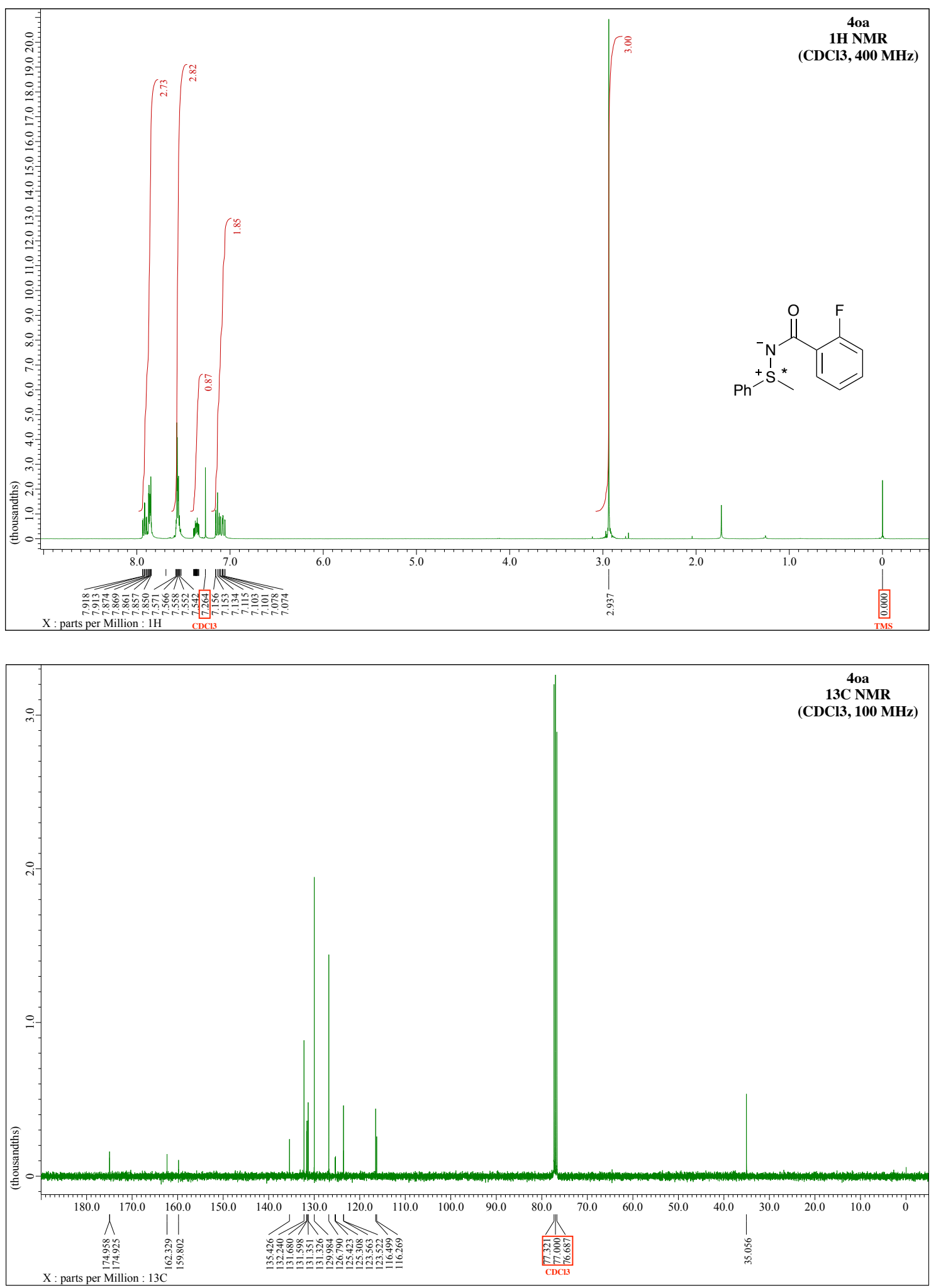
5.16. (R)-(-)-N-Acetyl methyl phenyl sulfimide (4pa)
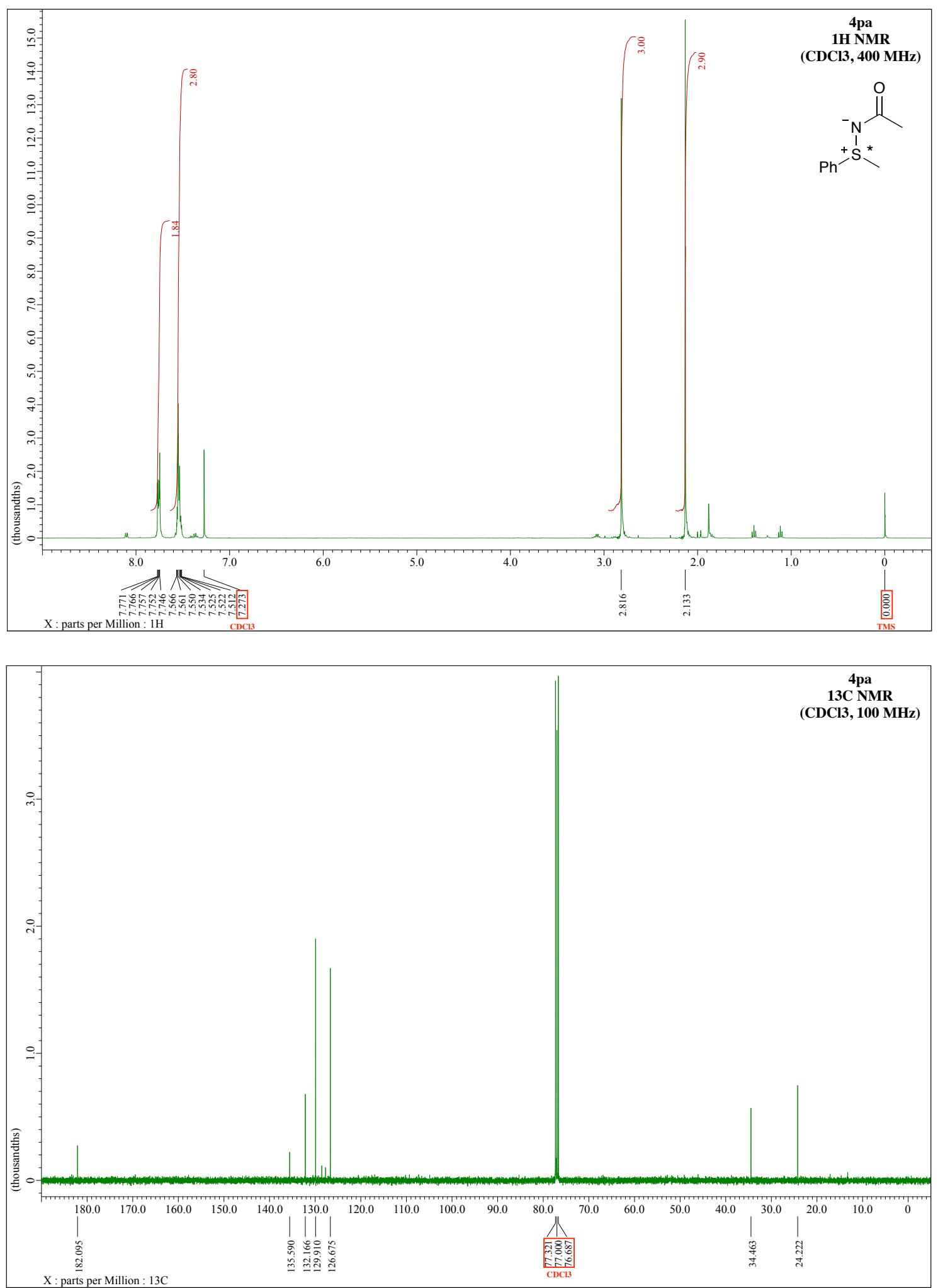
5.17. (-)- $N$-Hexanoyl methyl phenyl sulfimide (4qa)
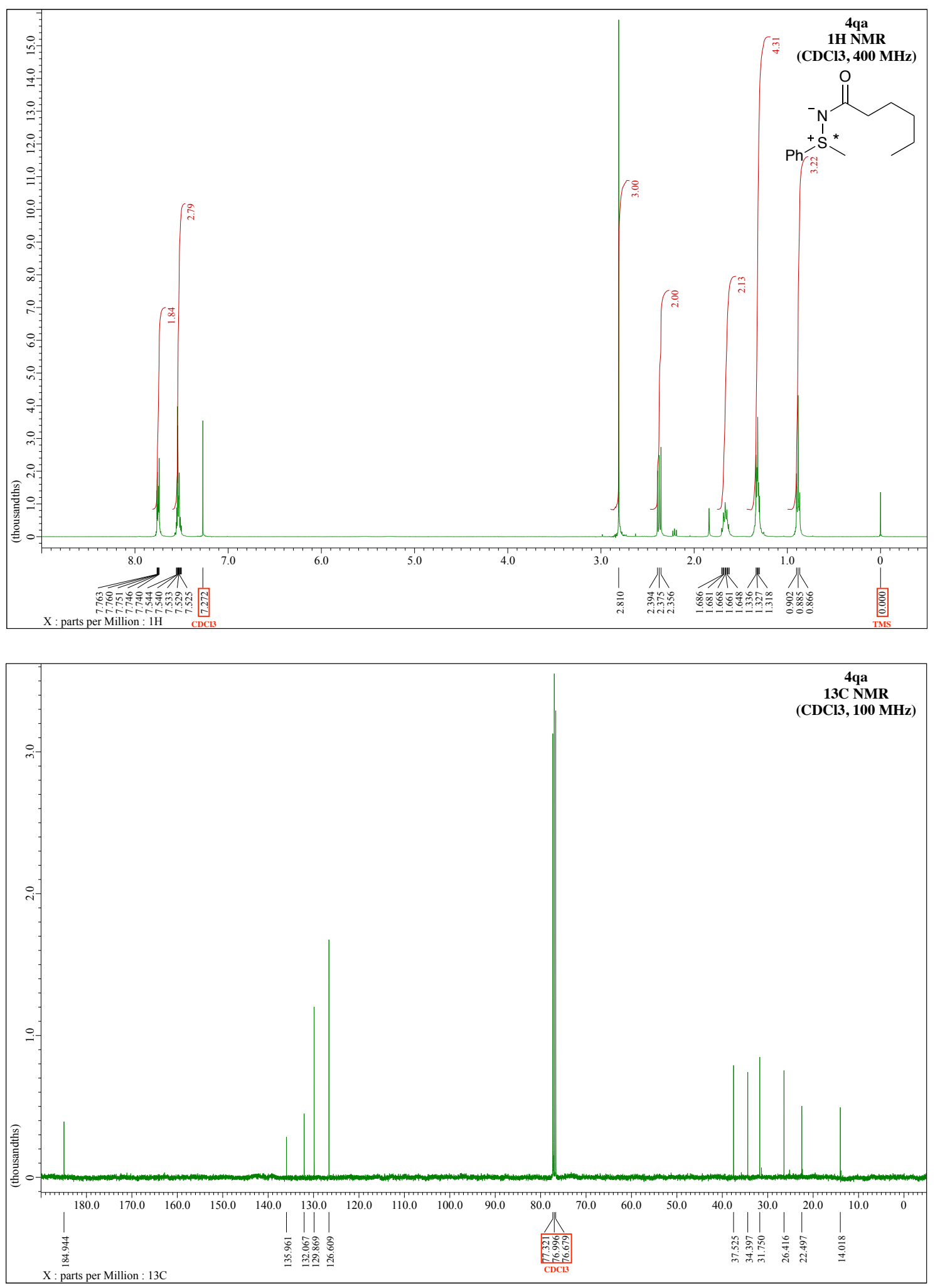
5.18. (-)- $N$-Isobutyloyl methyl phenyl sulfimide (4ra)
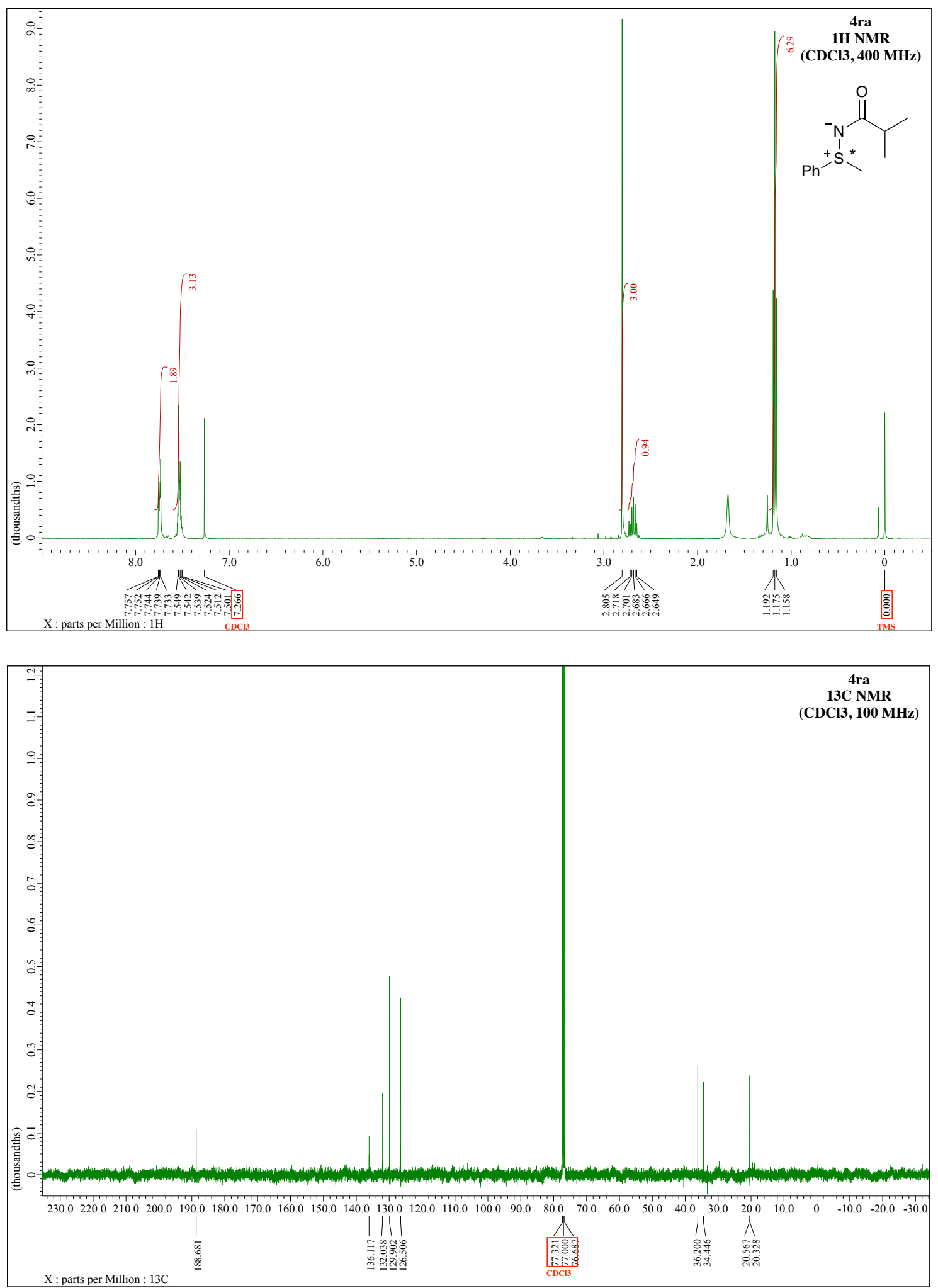
5.19. (-)-(E)-N-Cinnamoyl methyl phenyl sulfimide (4ta)
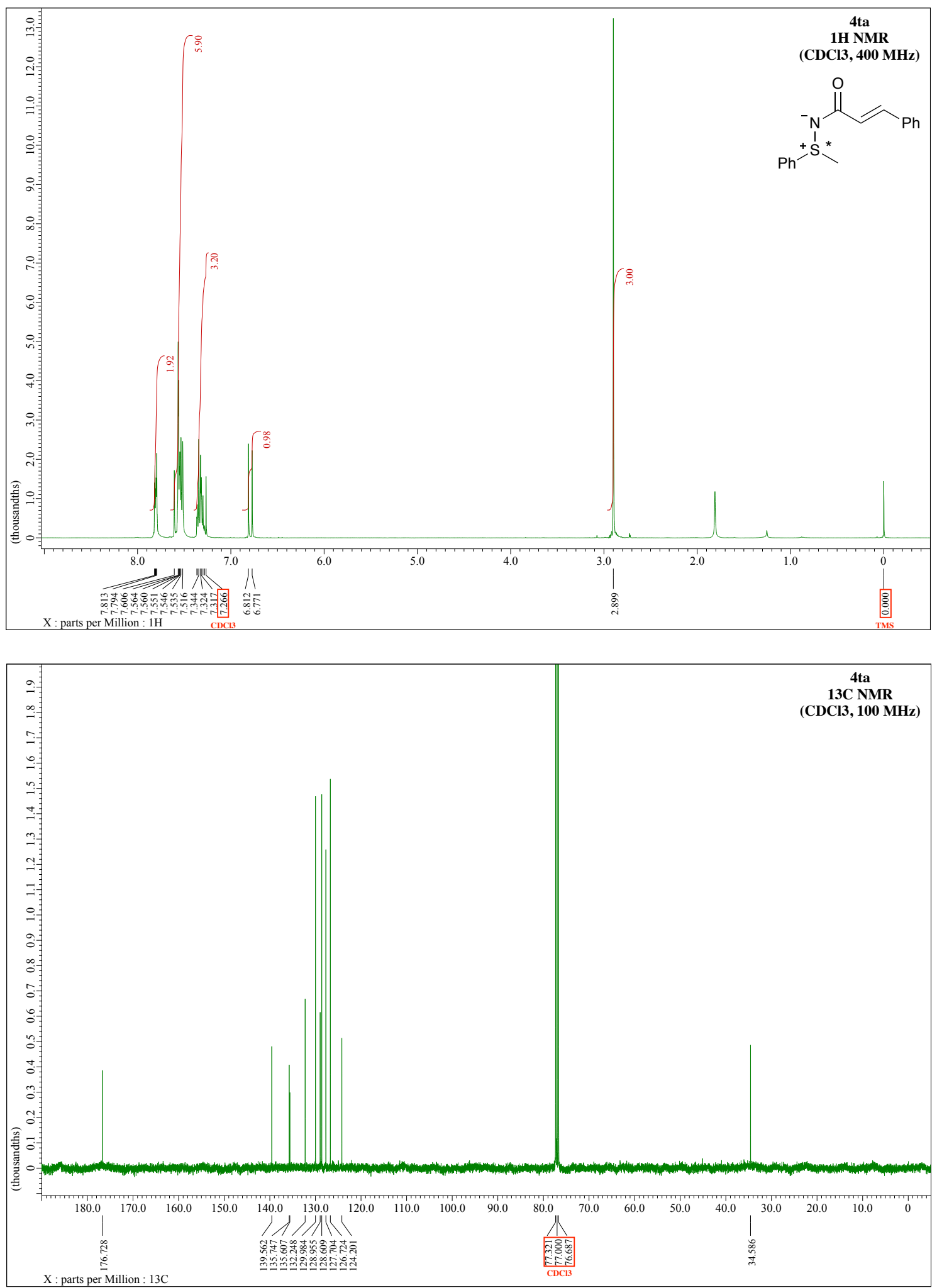
5.20. (-)- $N$-Benzoyl methyl 4-methylphenyl sulfimide (4ab)
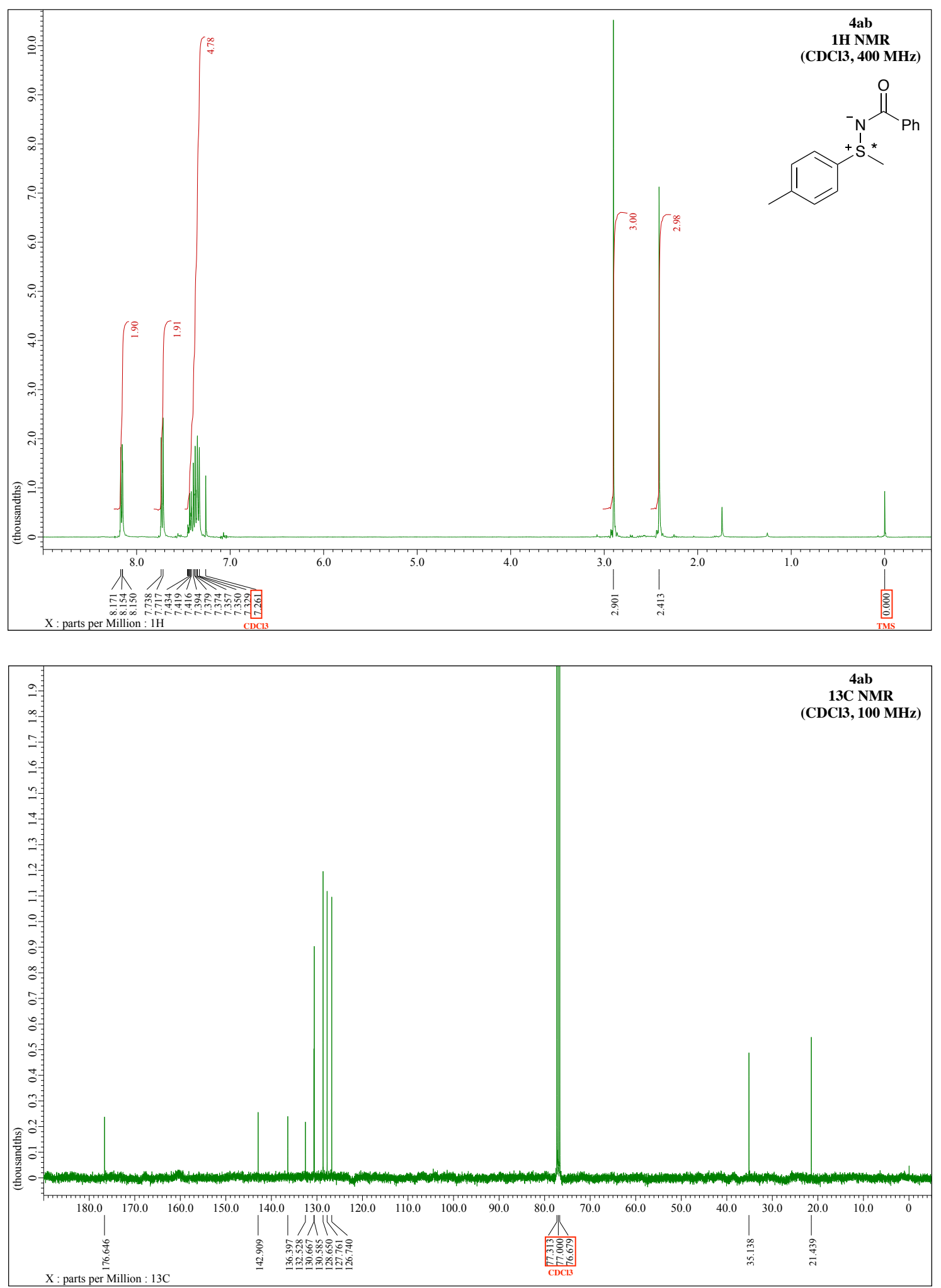
5.21. (-)-N-Benzoyl 4-methoxyphenyl methyl sulfimide (4ac)
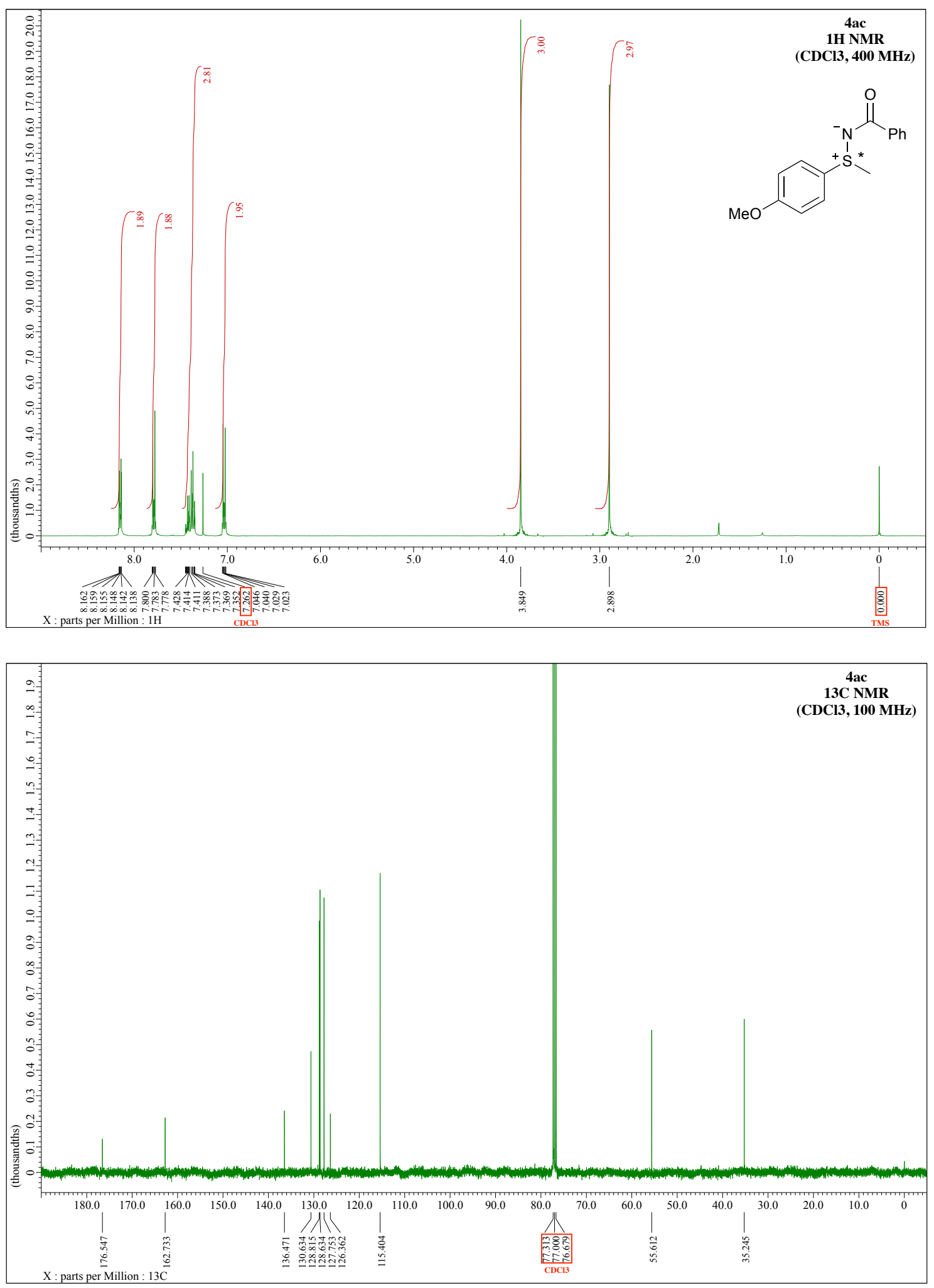
5.22. (-)- $N$-Benzoyl 4-bromophenyl methyl sulfimide (4ad)
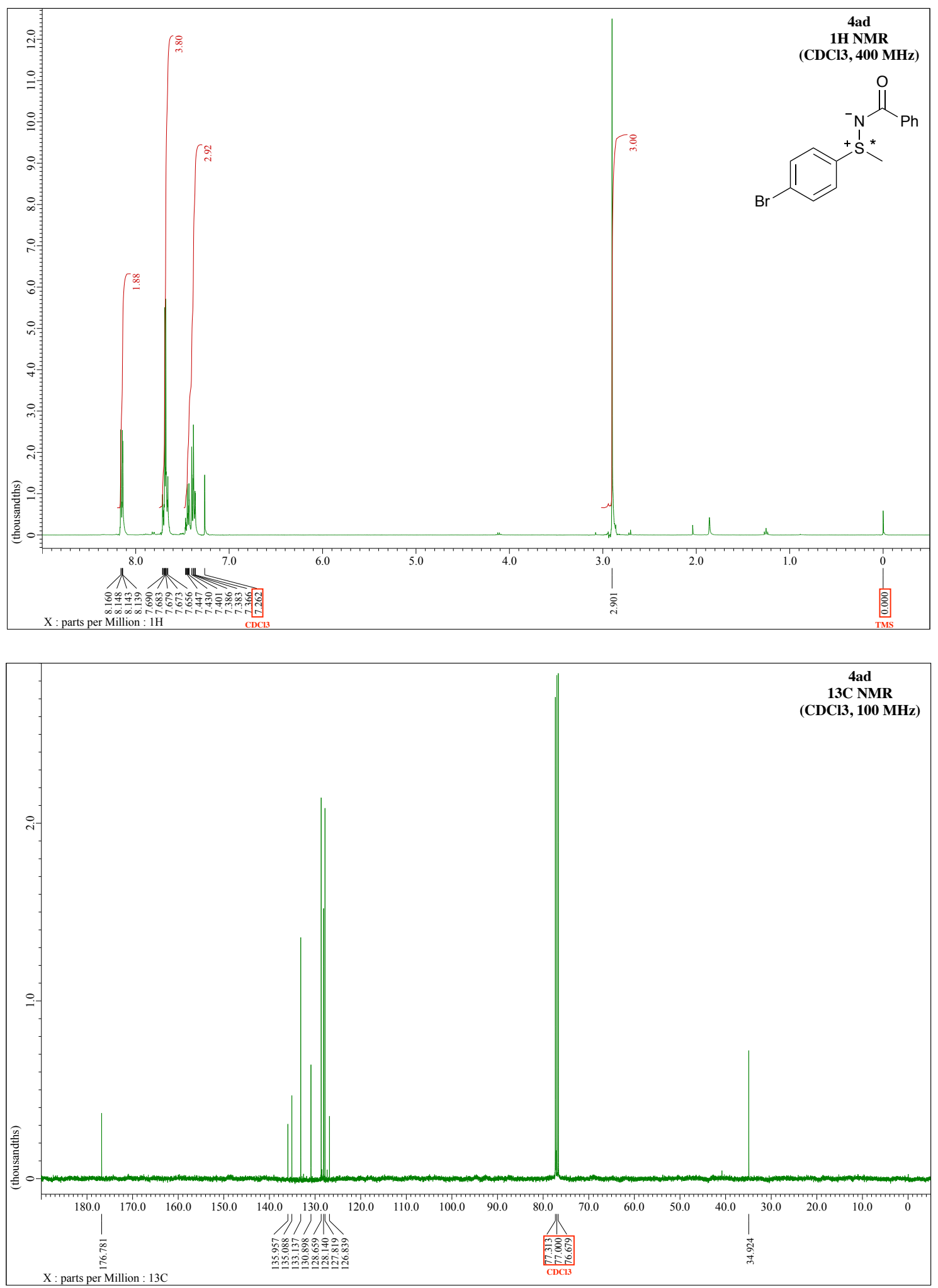
5.23. (-)-N-Benzoyl 4-chlorophenyl methyl sulfimide (4ae)
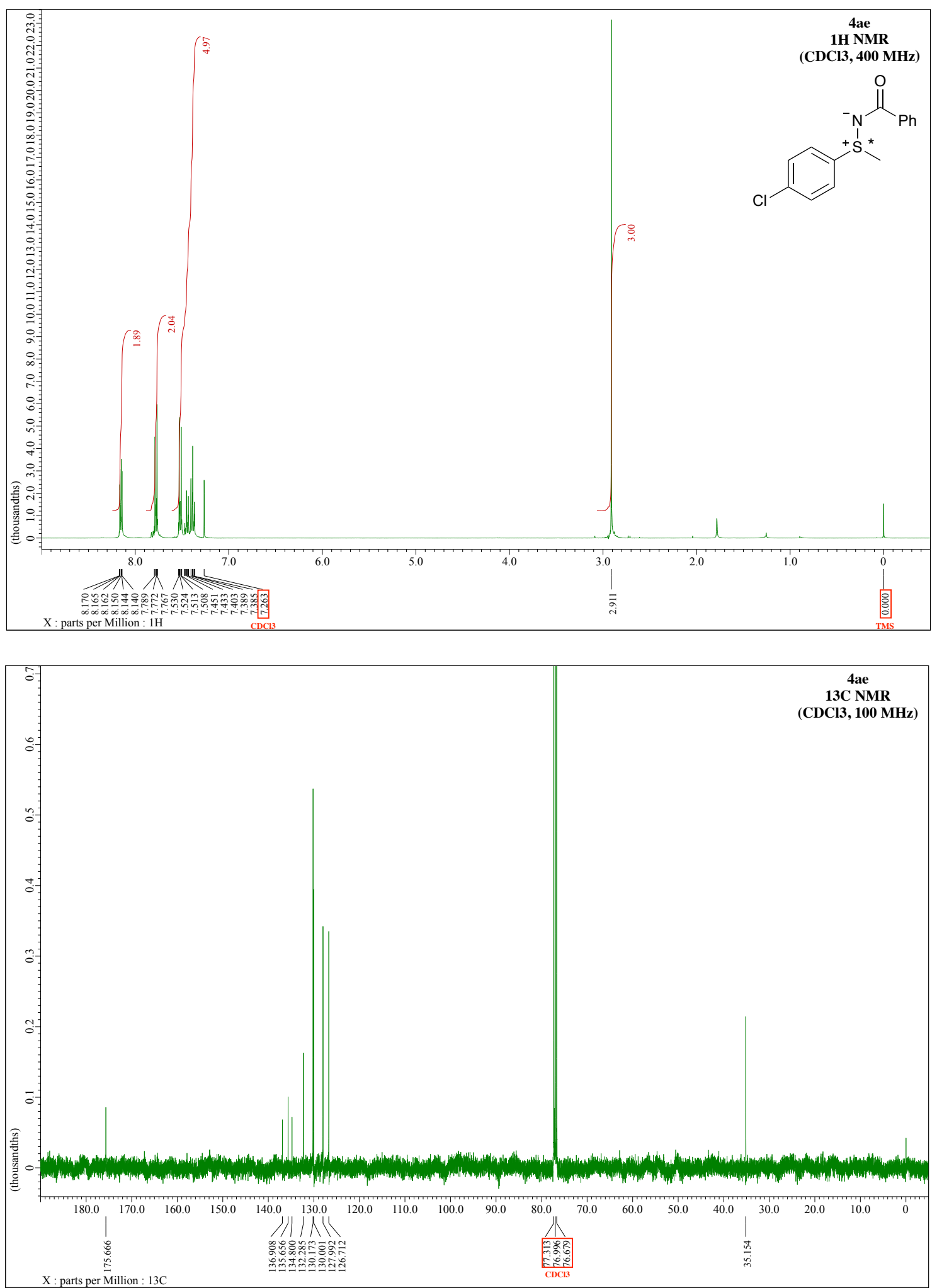
5.24. (-)-N-Benzoyl 4-fluorophenyl methyl sulfimide (4af)
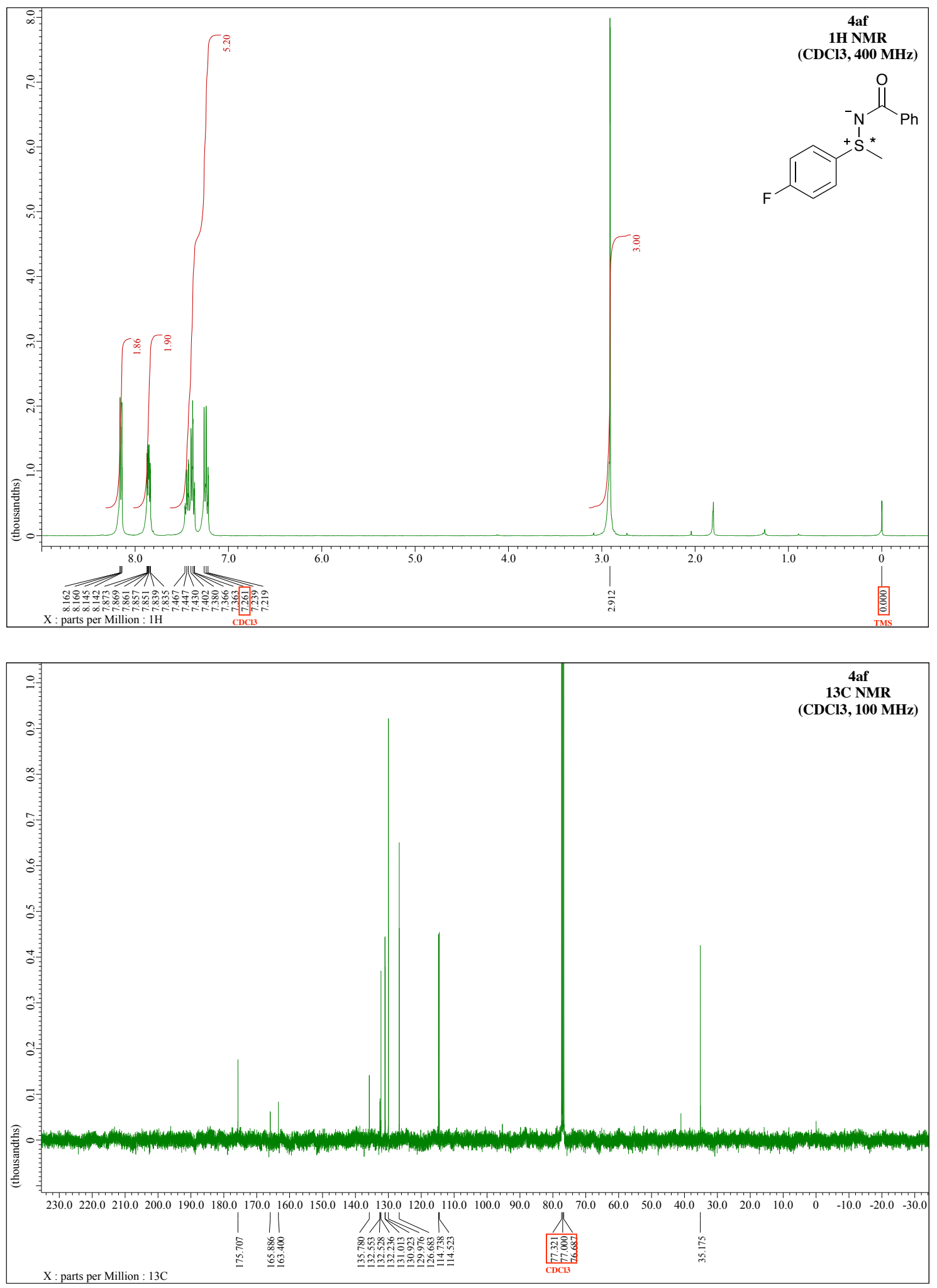
5.25. (-)- $N$-Benzoyl methyl 4-nitrophenyl sulfimide (4ag)
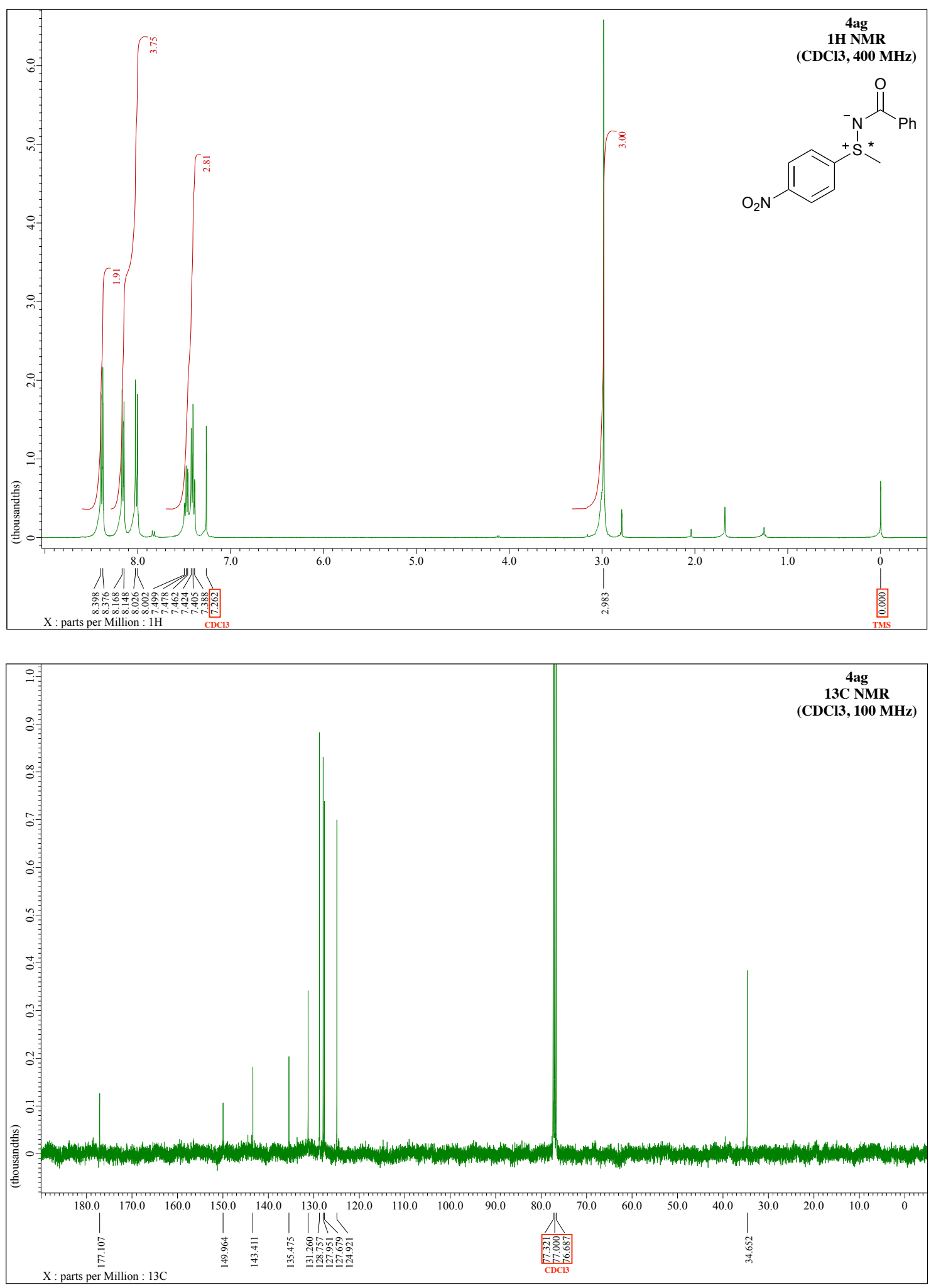
5.26. (-)- $N$-Benzoyl 3-bromophenyl methyl sulfimide (4ah)
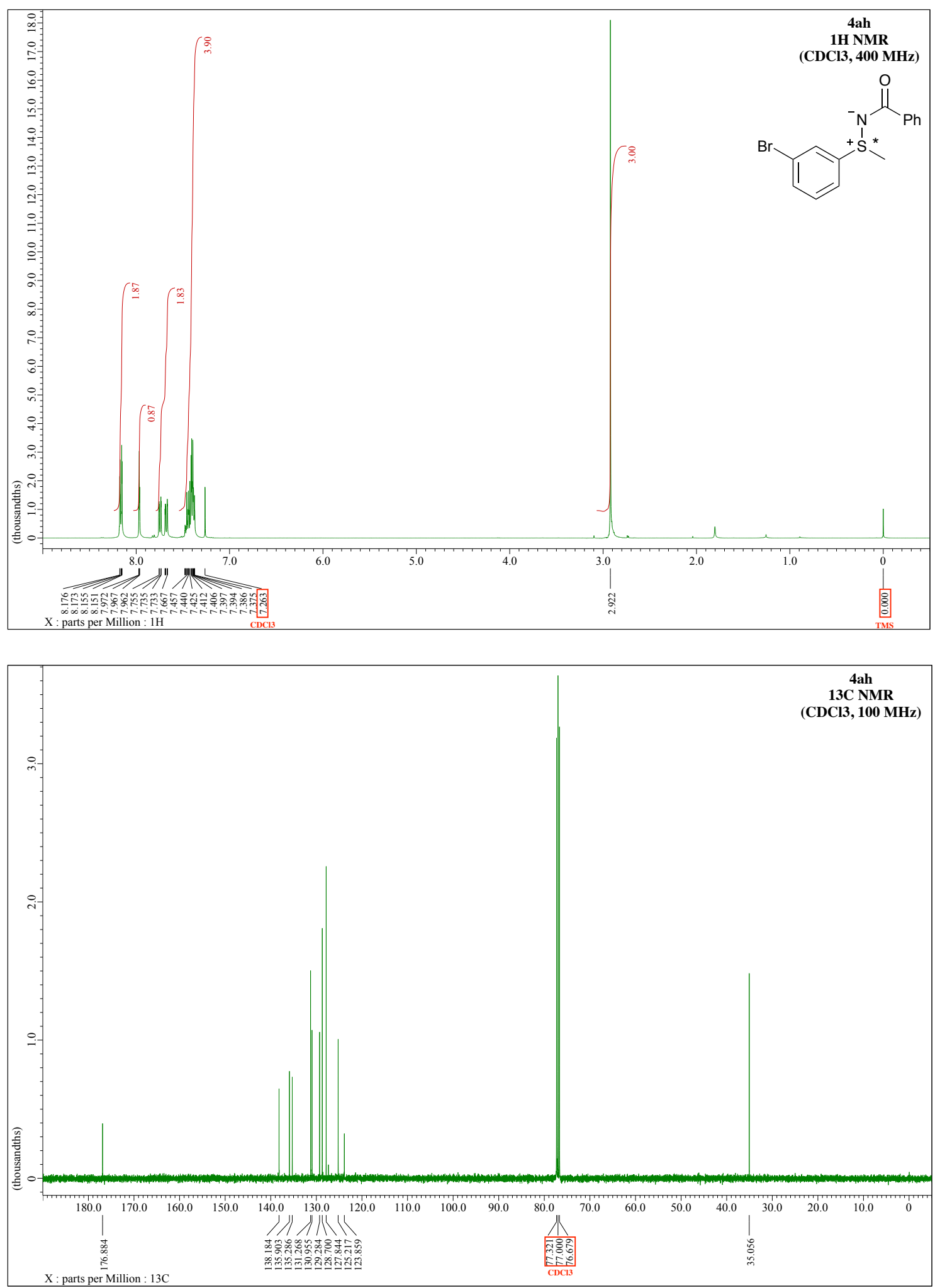
5.27. (-)- $N$-Benzoyl 2-bromophenyl methyl sulfimide (4ai)
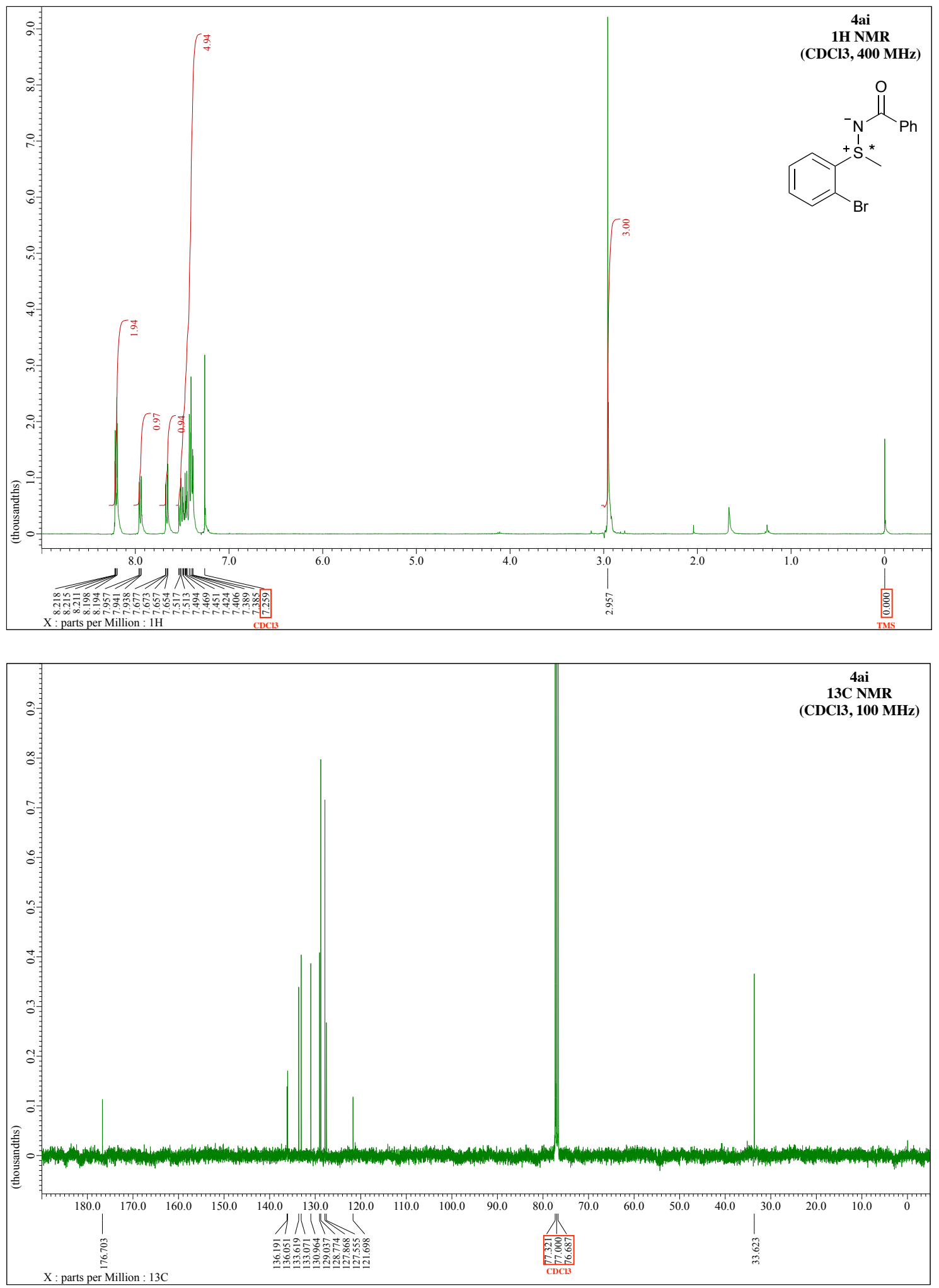
5.28. (-)- $N$-Benzoyl ethyl phenyl sulfimide (4aj)
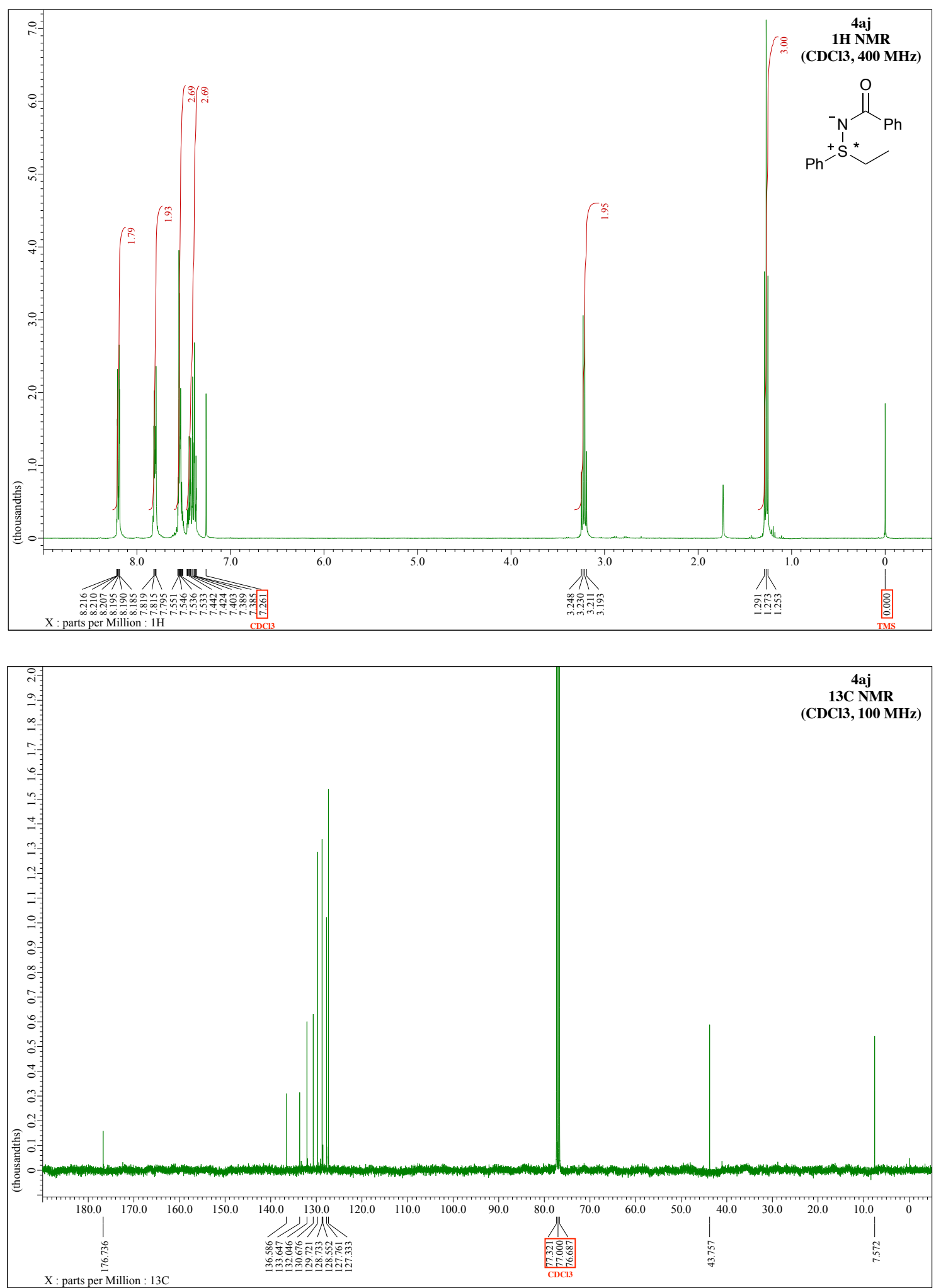
5.29. (-)- $N$-Benzoyl benzyl methyl sulfimide (4al)
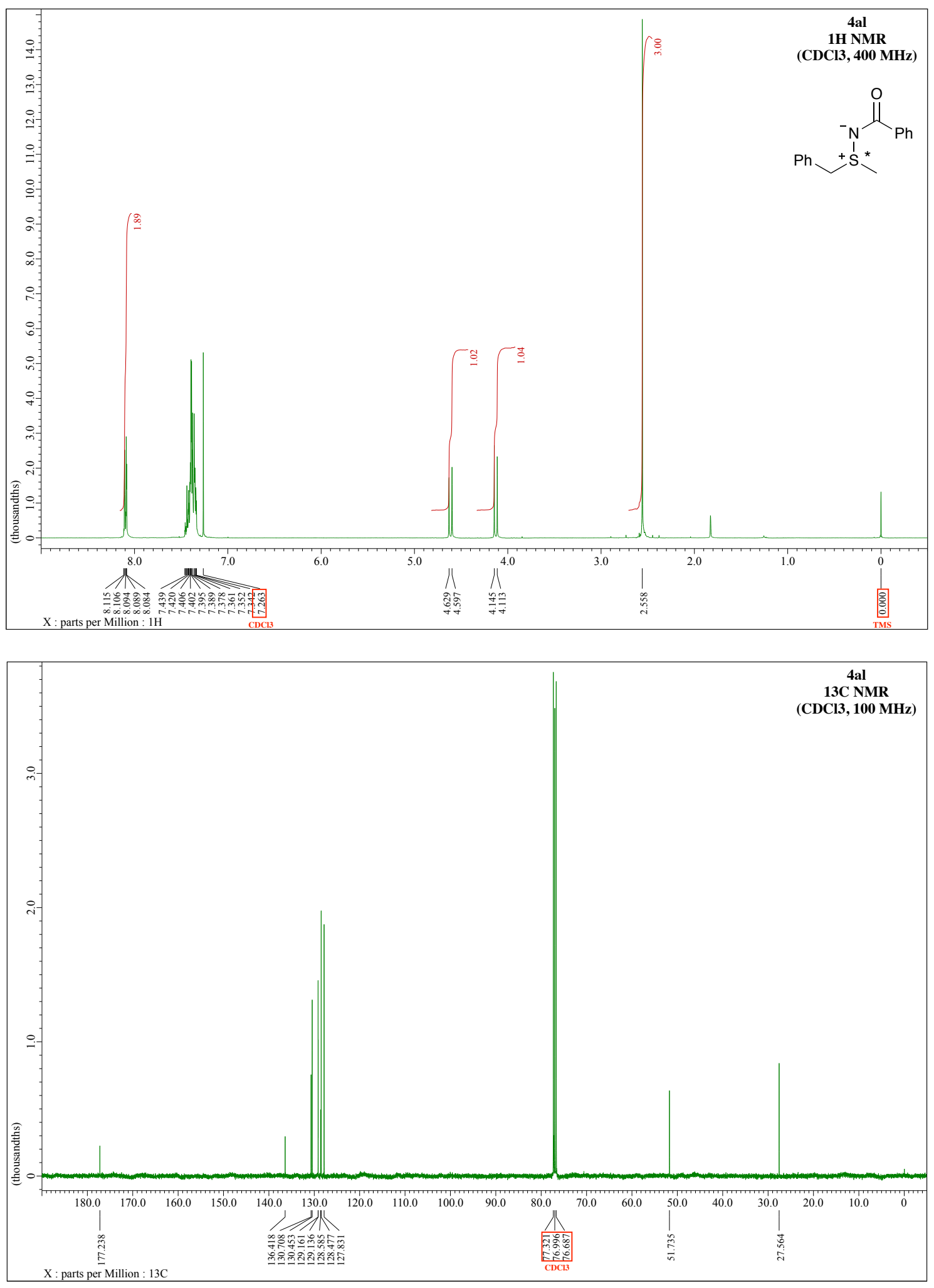
5.30. (+)- $N$-Benzoyl methyl octyl sulfimide (4am)
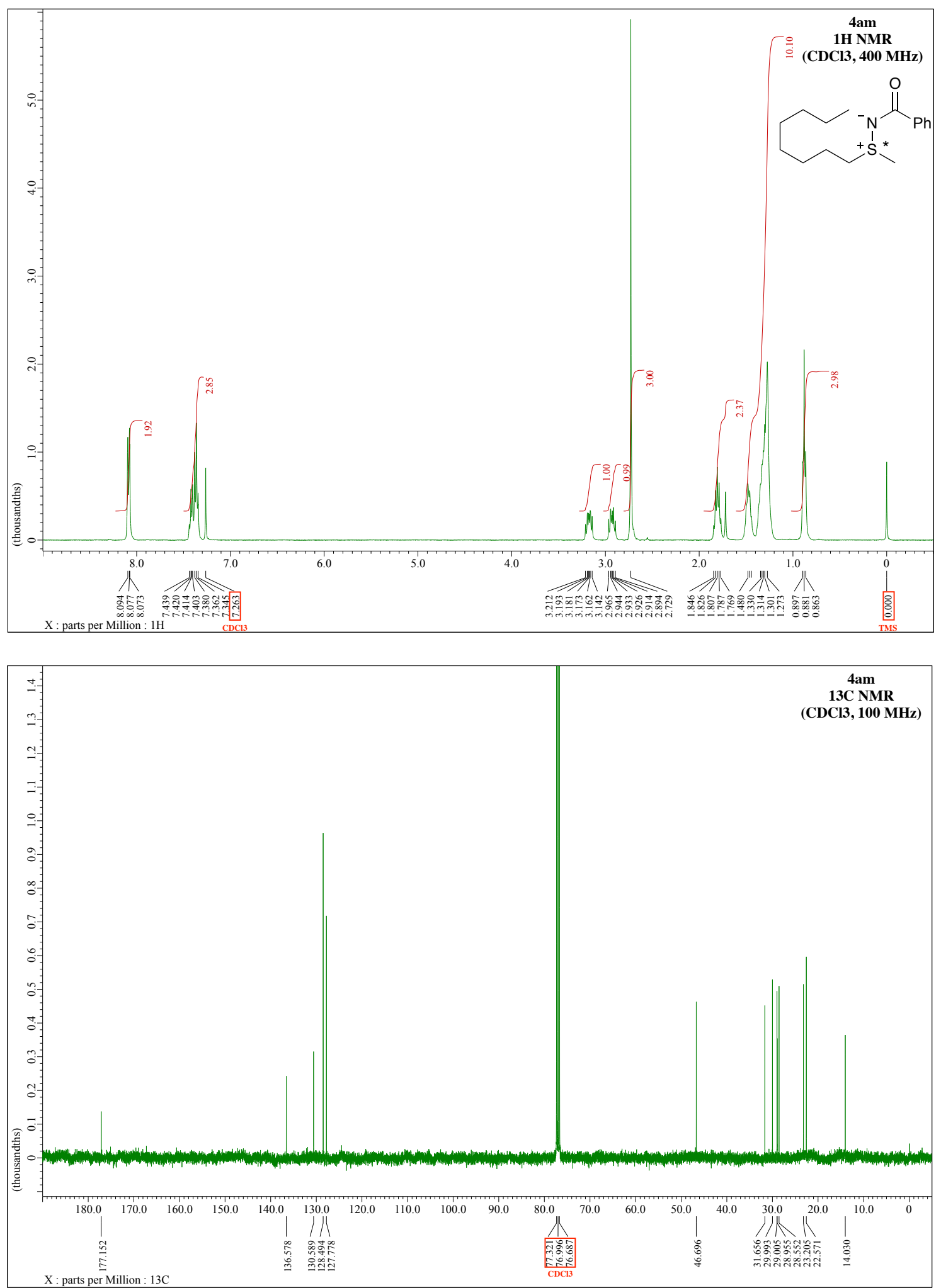
5.31. (+)- $N$-Benzoyl methyl 2-propyl sulfimide (4an)
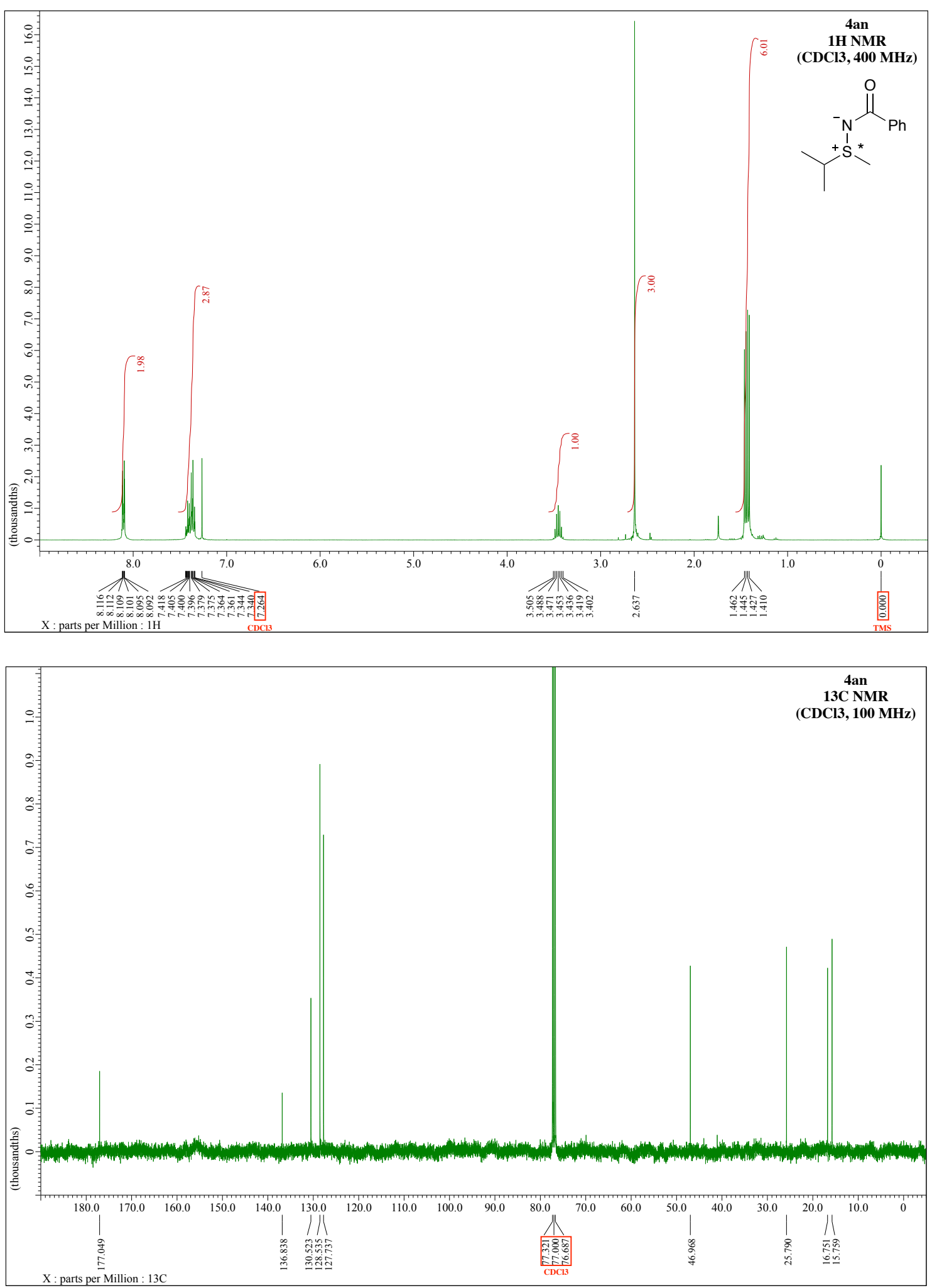
5.32. (+)- $N$-Benzoyl tert-butyl methyl sulfimide (4ao)
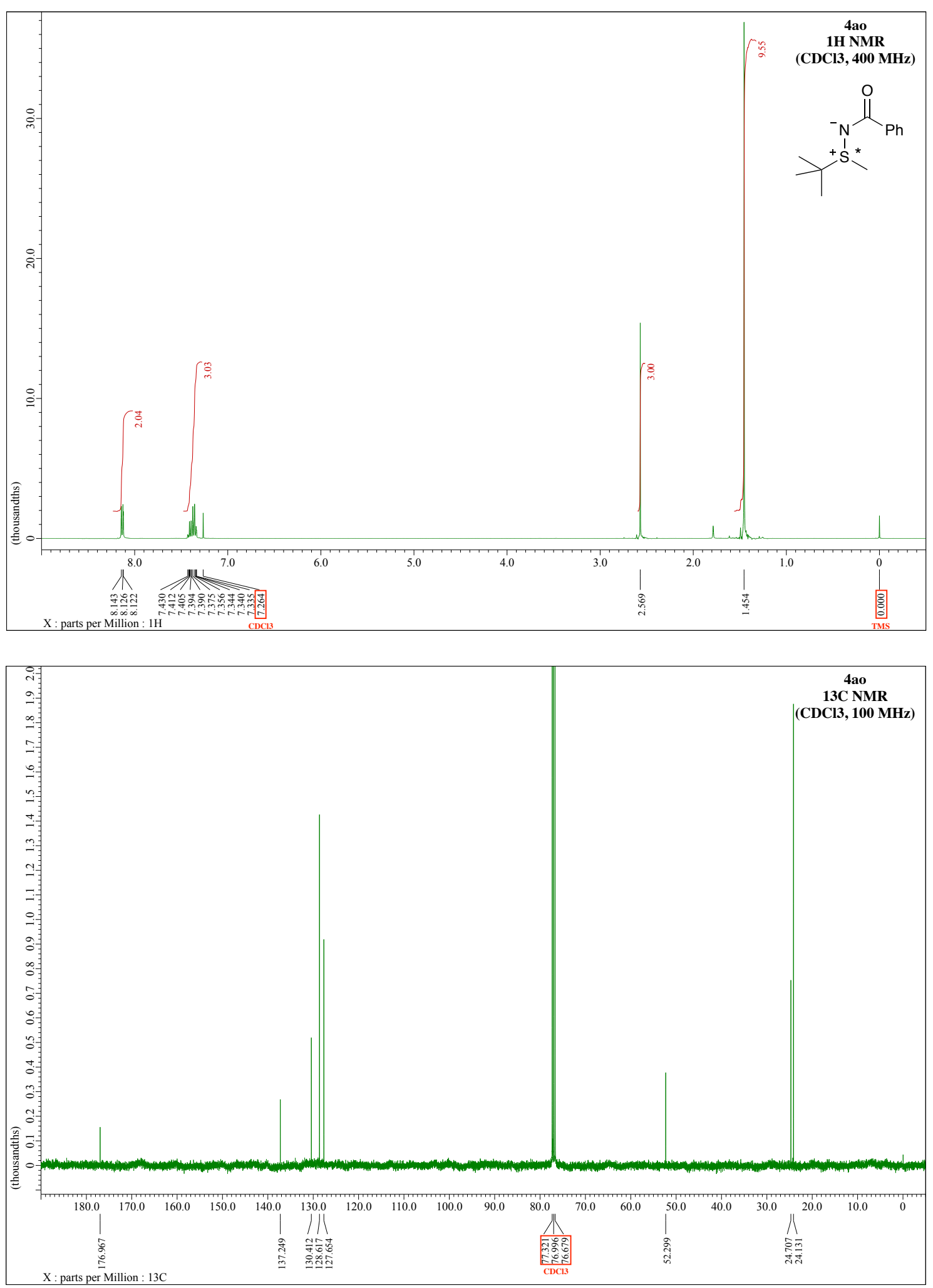


\subsection{3. (-)- $N$-Benzoyl ethyl propyl sulfimide (4ap)}
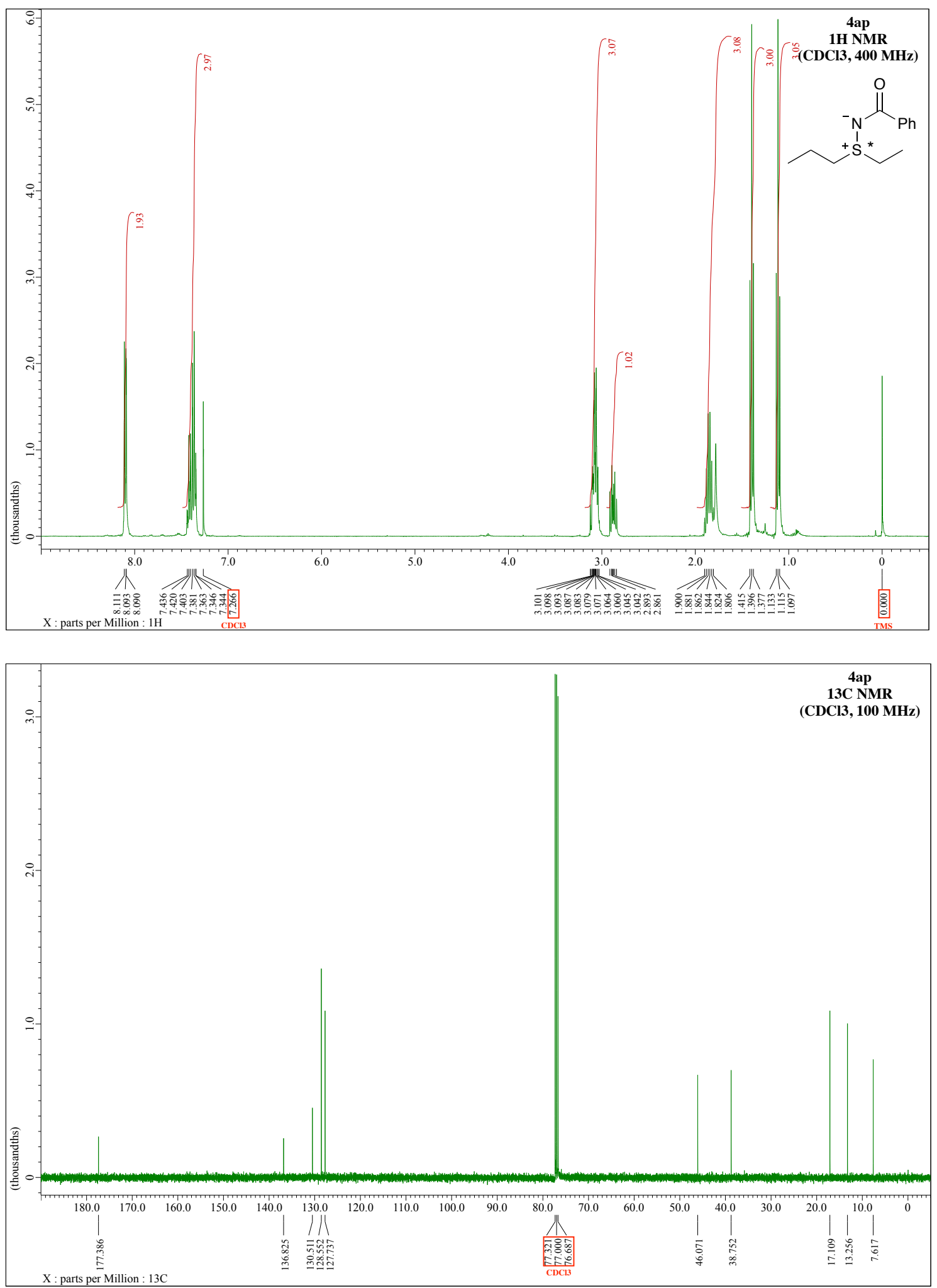
5.34. (+)- $N$-Benzoyl methyl phenyl sulfoximine (5aa)
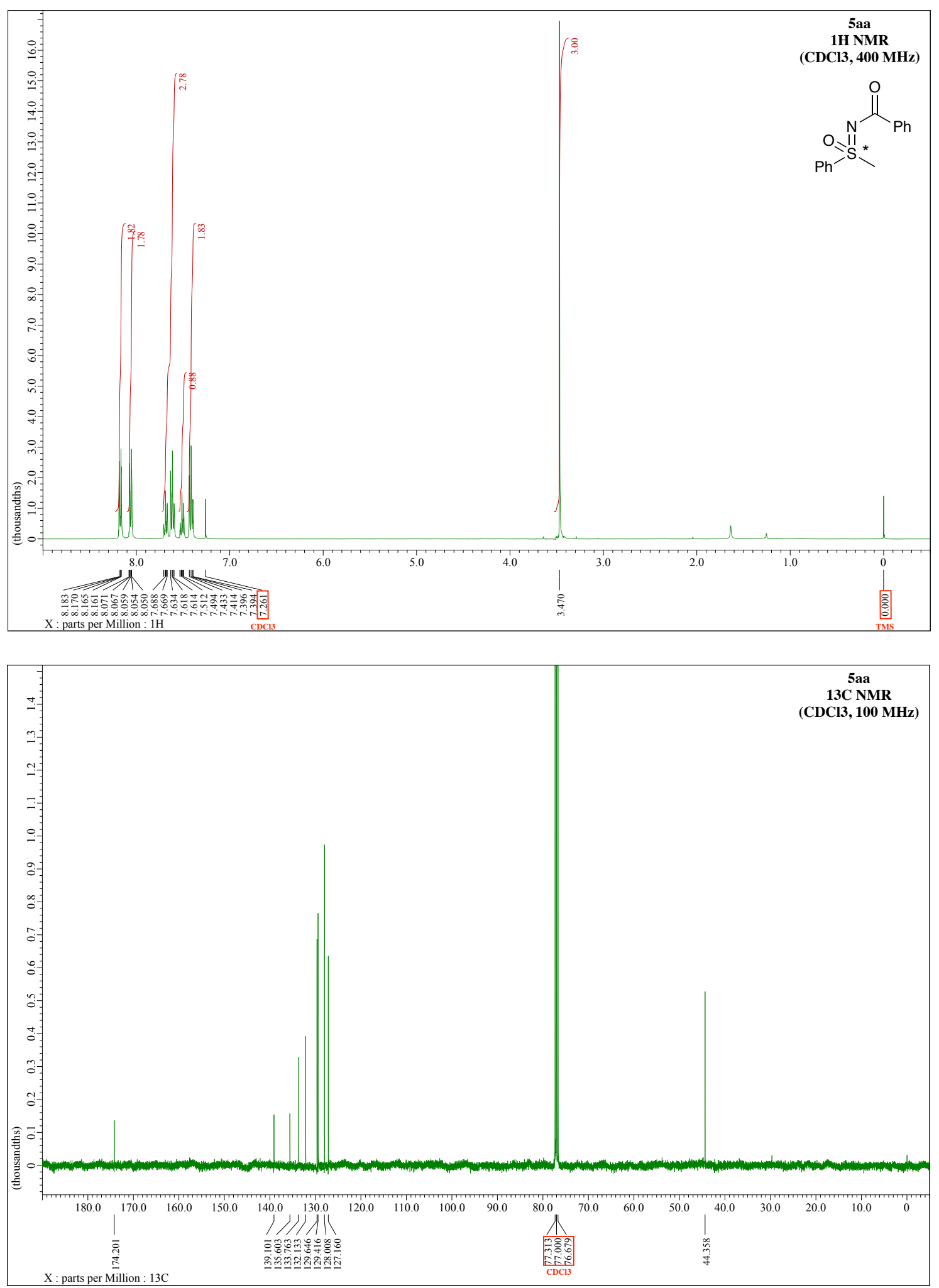
5.35. (R)-(-)- $N$-Acyl methyl phenyl sulfoximine (5pa)
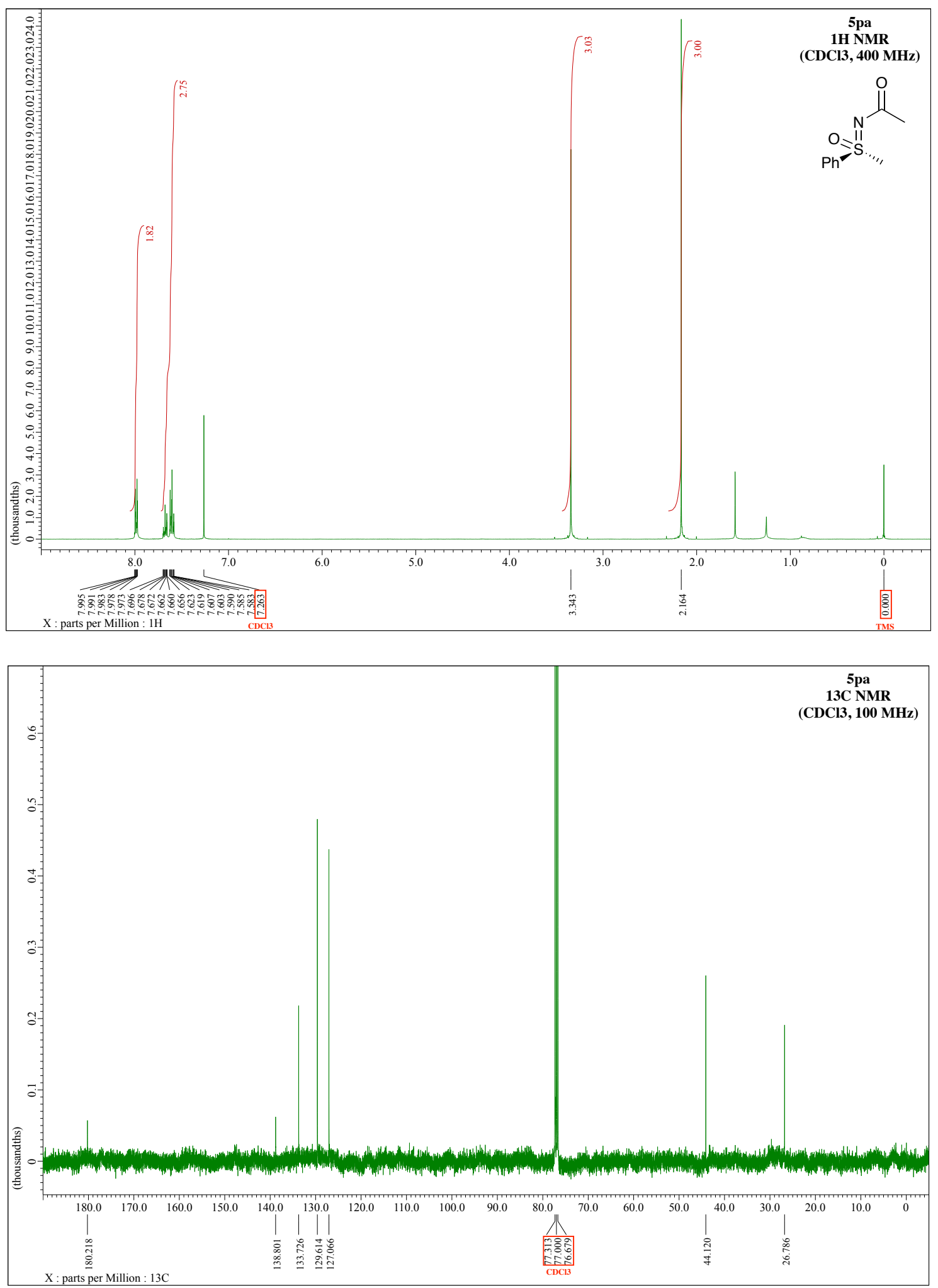
6. The Chromatograms for the determination of enantioselectivity.

6.1. (-)- $N$-Benzoyl methyl phenyl sulfimide (4aa)
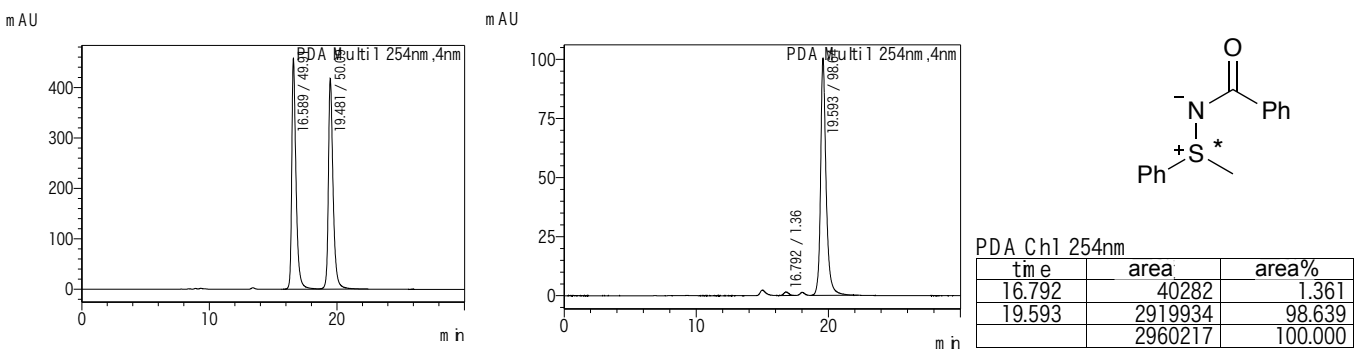

6.2. (-)- $N$-(4-Methylbenzoyl) methyl phenyl sulfimide (4ba)
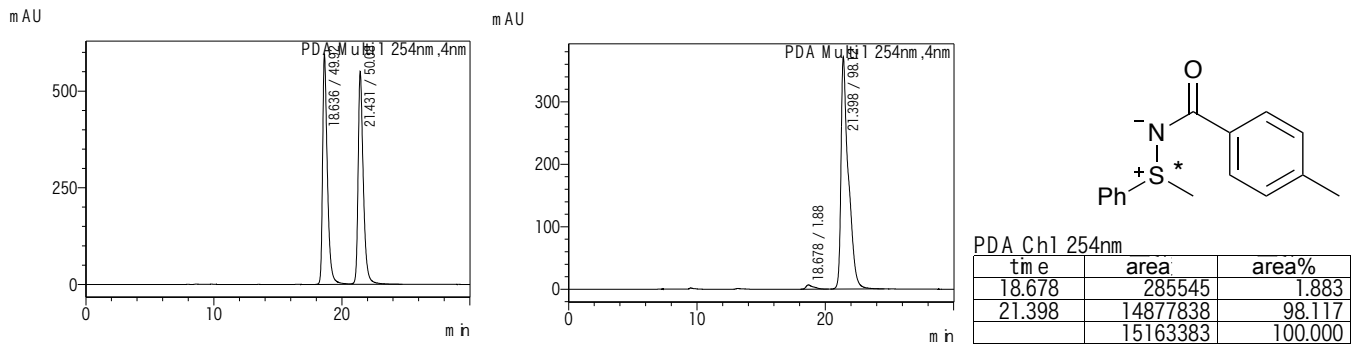

6.3. (-)-N-(4-Methoxylbenzoyl) methyl phenyl sulfimide (4ca)
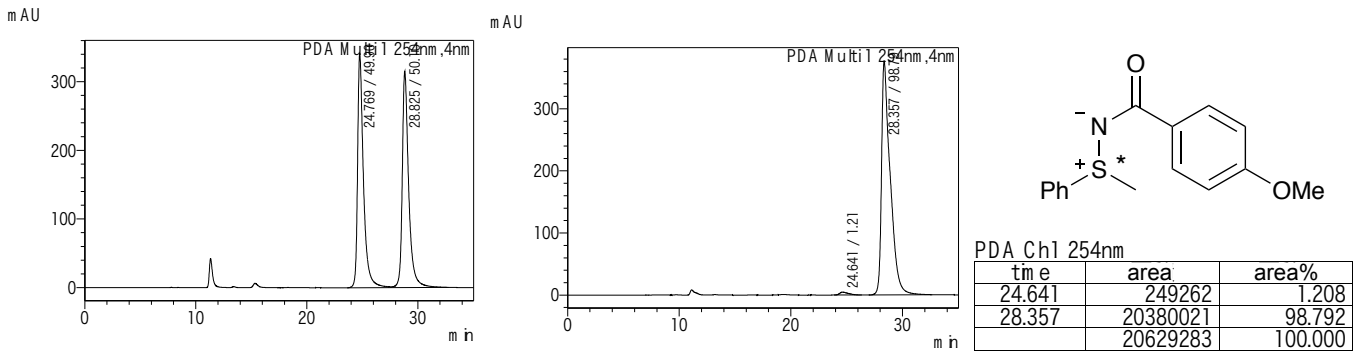

6.4. (-)- $N$-(4-Bromobenzoyl) methyl phenyl sulfimide (4da)
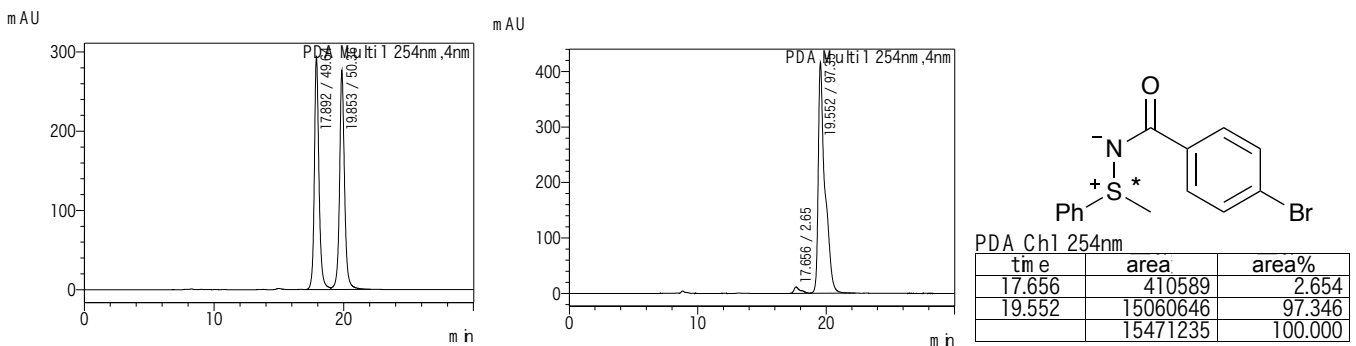
6.5. (-)- $N$-(4-Chlorobenzoyl) methyl phenyl sulfimide (4ea)
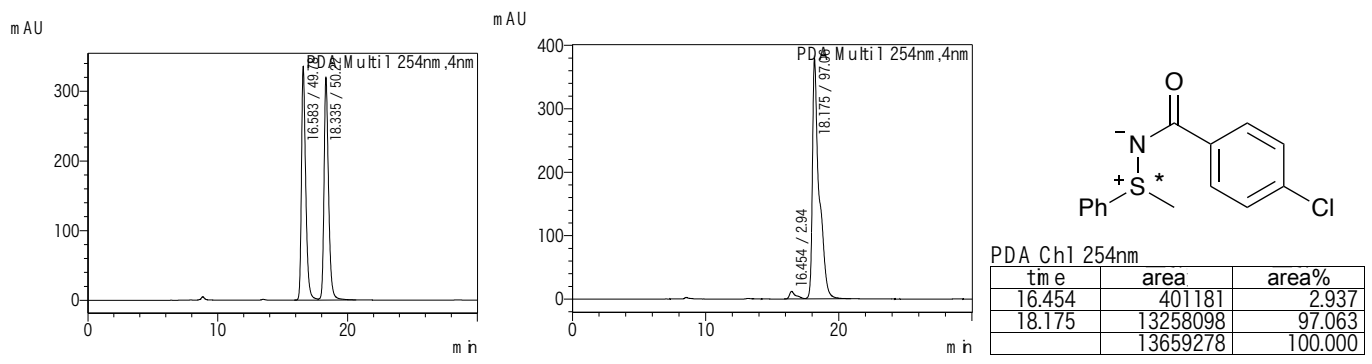

6.6. (-)- $N$-(4-Fluorobenzoyl) methyl phenyl sulfimide (4fa)
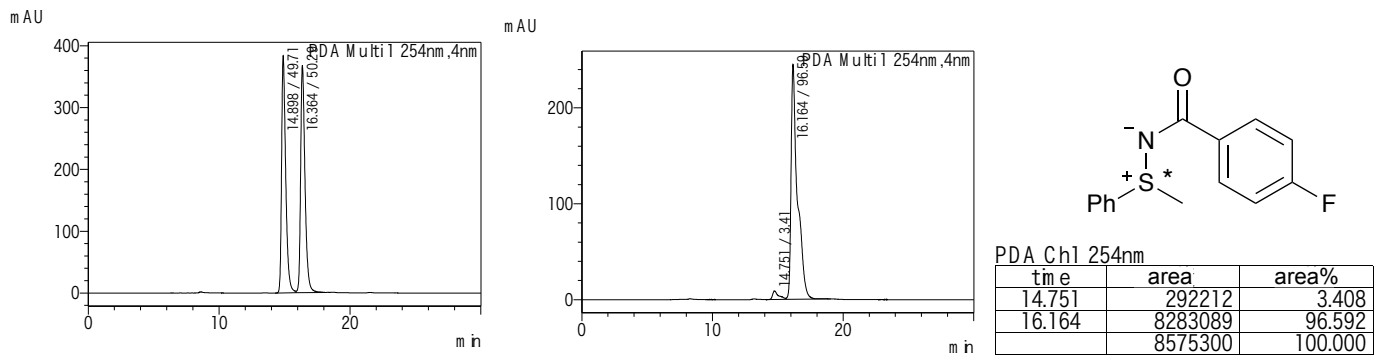

6.7. (-)- $N$-(4-Trifluoromethylbenzoyl) methyl phenyl sulfimide (4ga)
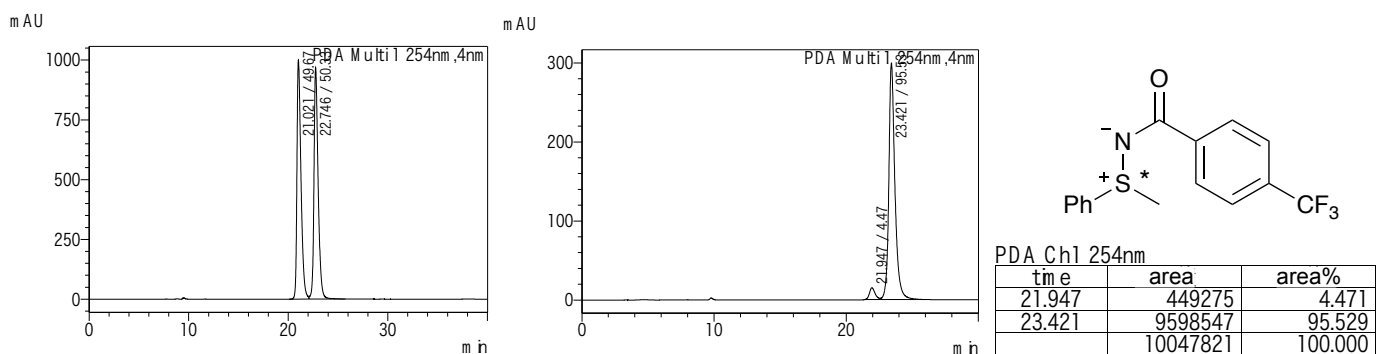

6.8. (-)- $N$-(3-Methylbenzoyl) methyl phenyl sulfimide (4ha)
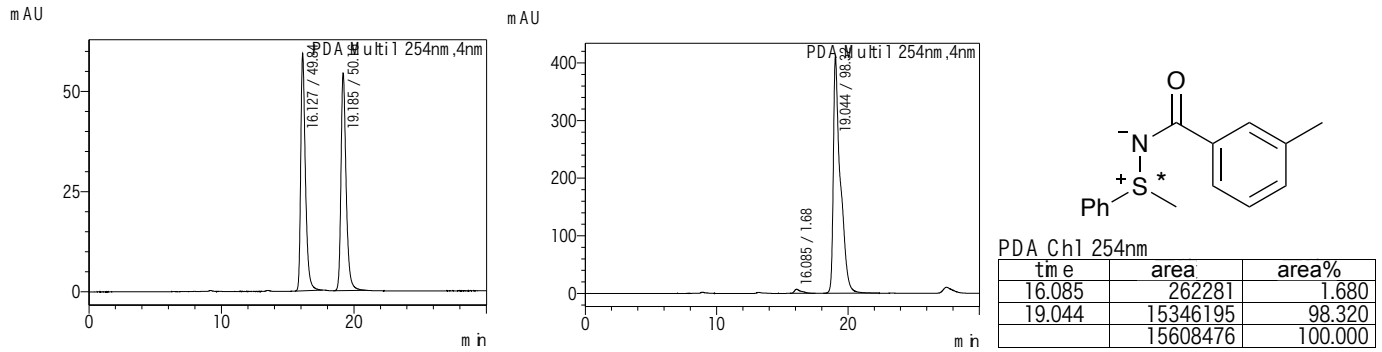
6.9. (-)- $N-(3-$ Bromobenzoyl) methyl phenyl sulfimide (4ia)
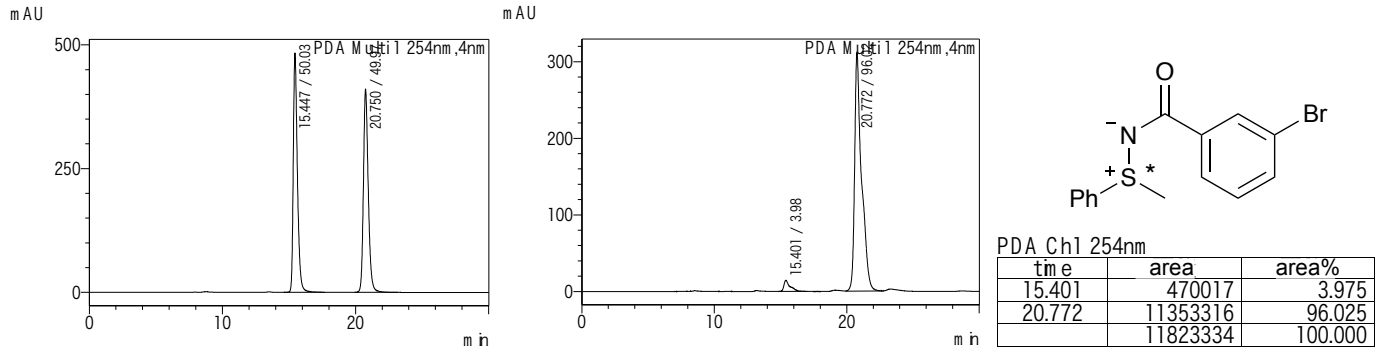

6.10. (-)- $N$-(3-Chlorobenzoyl) methyl phenyl sulfimide (4ja)
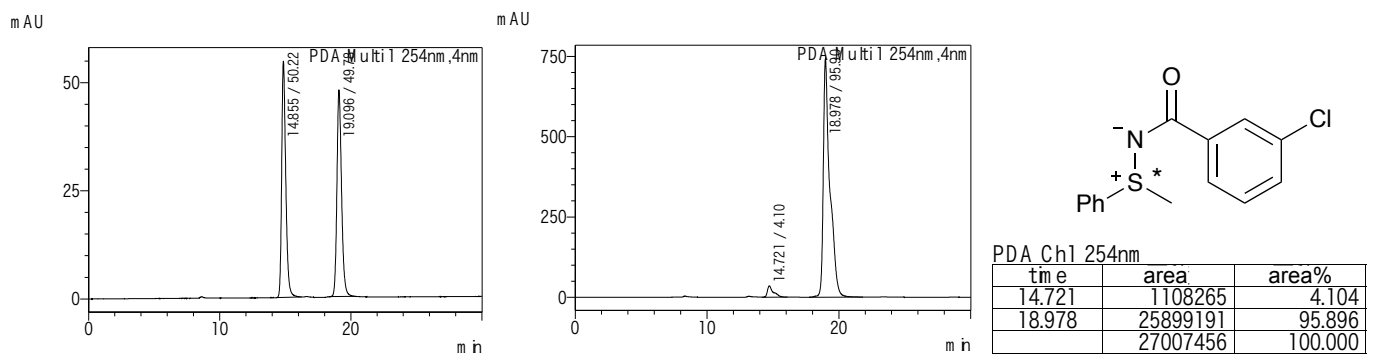

6.11. (-)- $N$-(3-Fluorobenzoyl) methyl phenyl sulfimide (4ka)
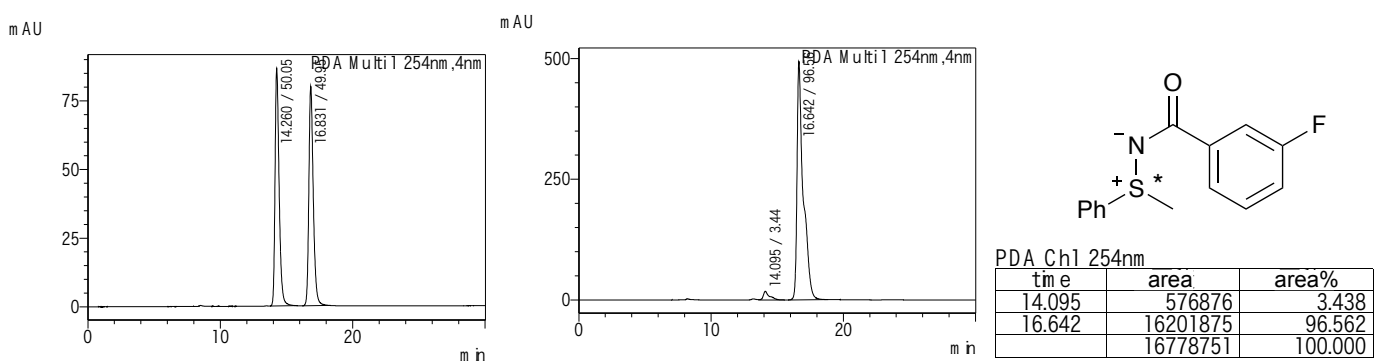

6.12. (-)- $N$-(2-Methylbenzoyl) methyl phenyl sulfimide (4la)

$\mathrm{mAU}$

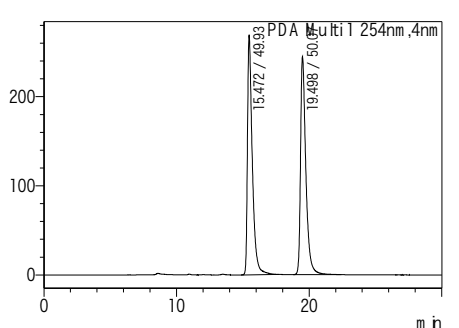

$\mathrm{mAU}$
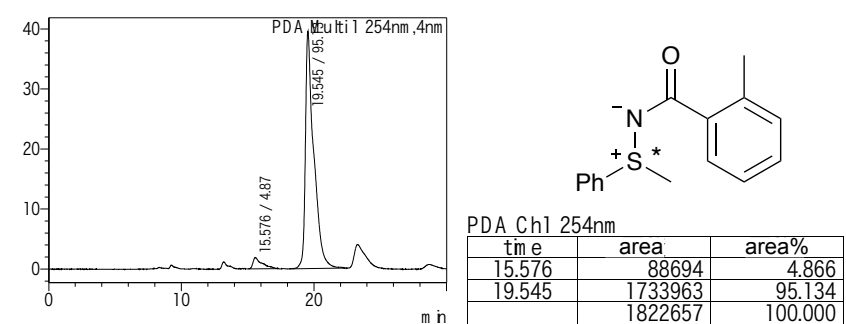
6.13. (-)-N-(2-Bromobenzoyl) methyl phenyl sulfimide (4ma)
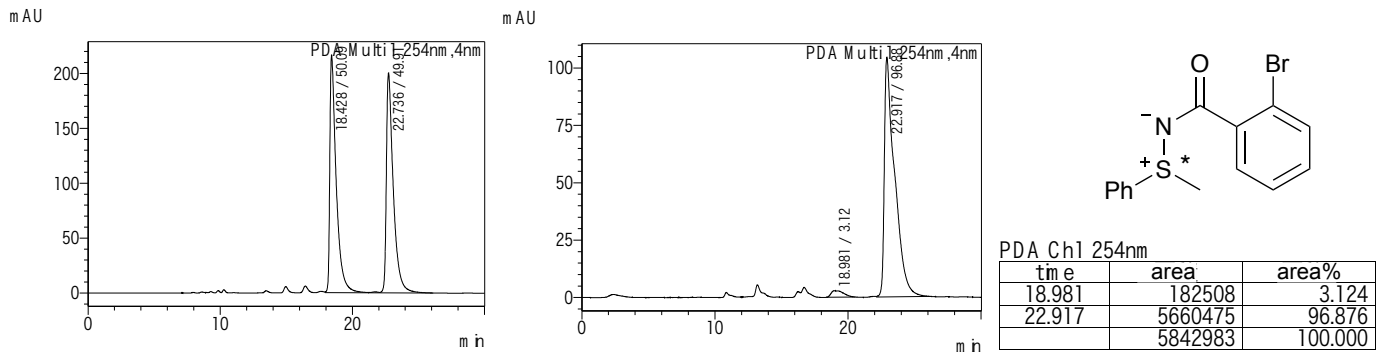

6.14. (-)-N-(2-Chlorobenzoyl) methyl phenyl sulfimide (4na)
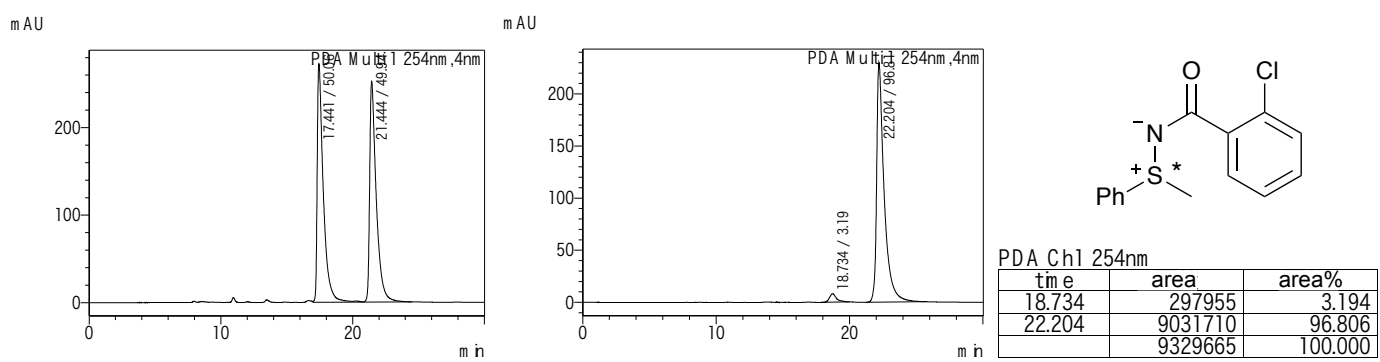

6.15. (-)- $N$-(2-Fluorobenzoyl) methyl phenyl sulfimide (40a)
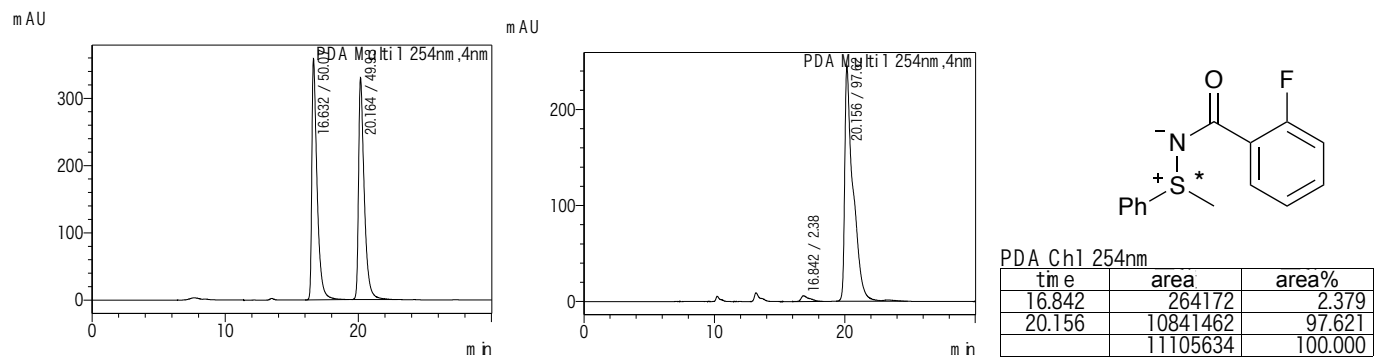

6.16. (R)-(-)-N-Acetyl methyl phenyl sulfimide (4pa)
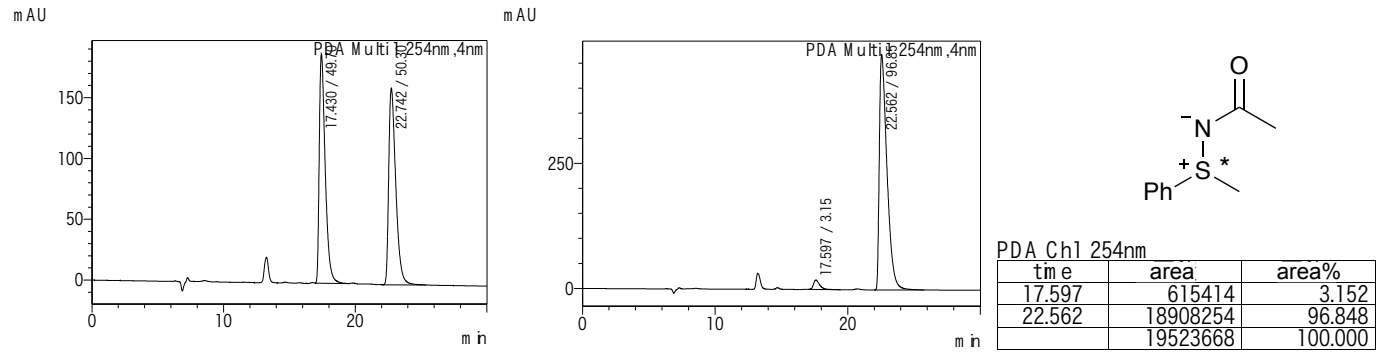
6.17. (-)-N-Hexanoyl methyl phenyl sulfimide (4qa)
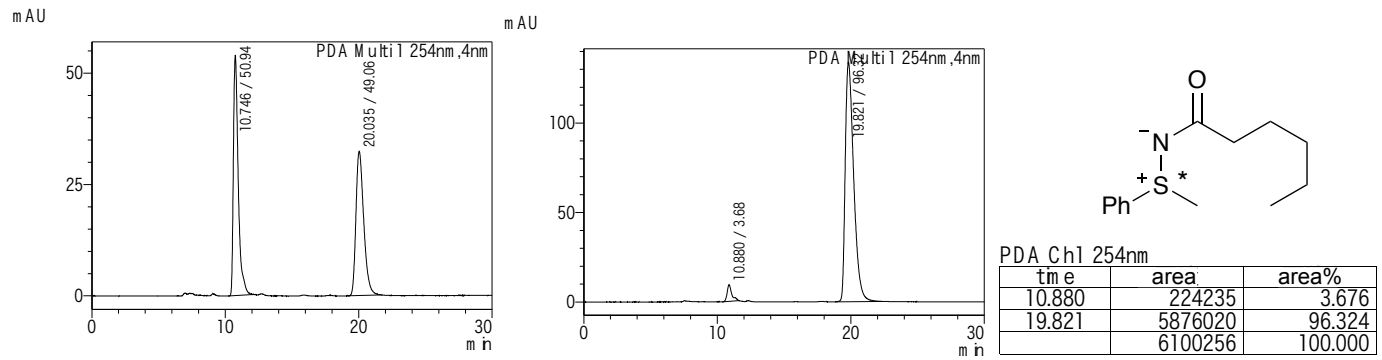

6.18. (-)- $N$-Isobutyloyl methyl phenyl sulfimide (4ra)
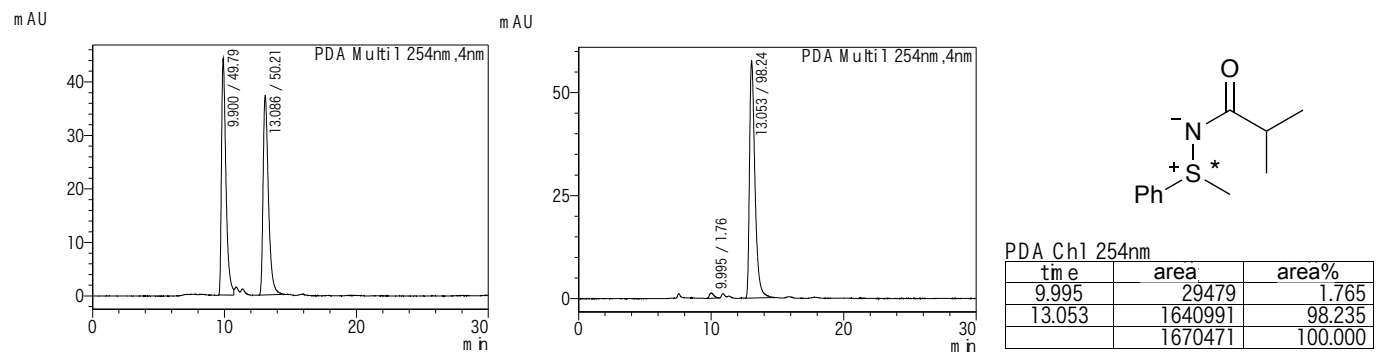

6.19. (-)-(E)- $N$-Cinnamoyl methyl phenyl sulfimide (4ta)
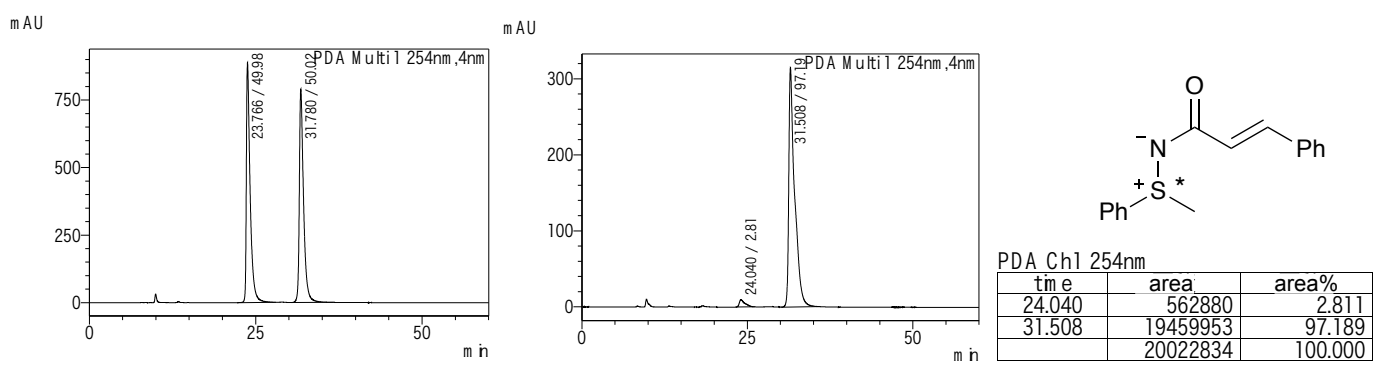

6.20. (-)- $N$-Benzoyl methyl 4-methylphenyl sulfimide (4ab)
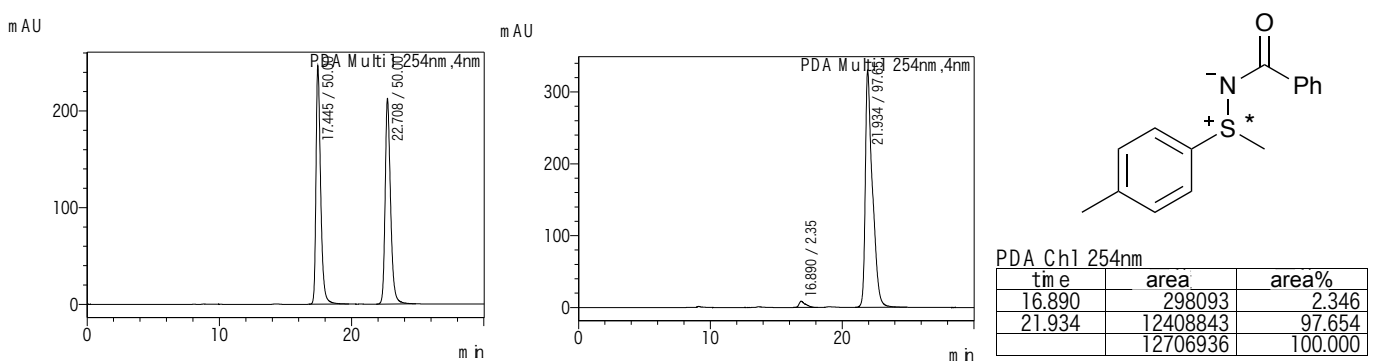
6.21. (-)- $N$-Benzoyl 4-methoxyphenyl methyl sulfimide (4ac)
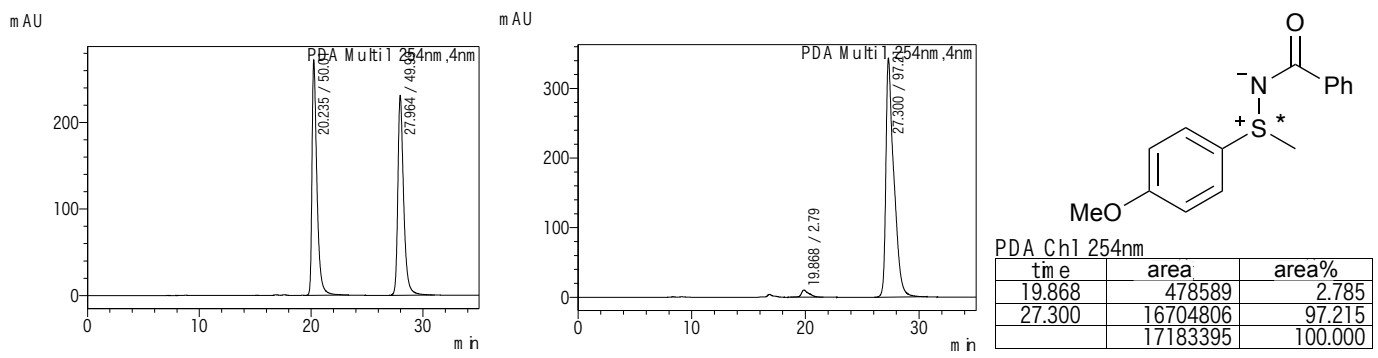

6.22. (-)- $N$-Benzoyl 4-bromophenyl methyl sulfimide (4ad)
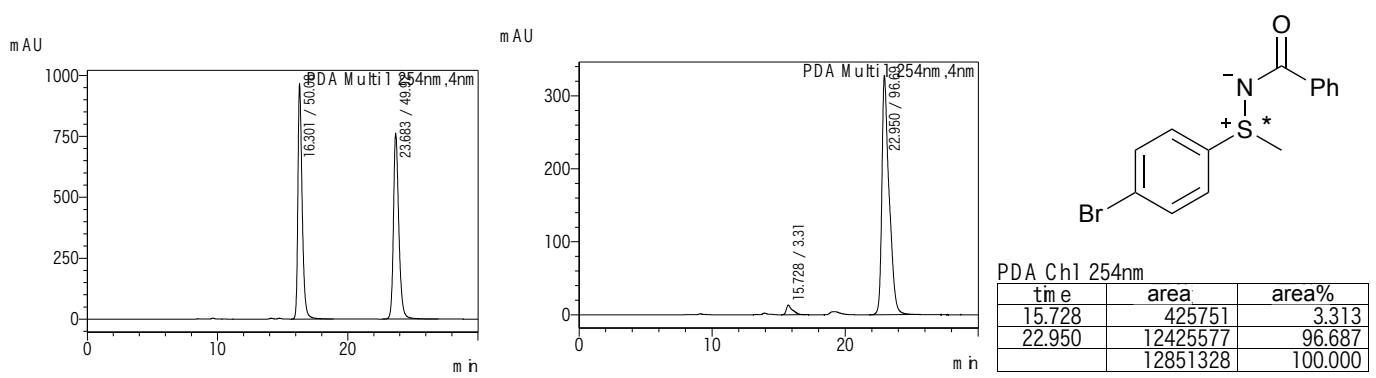

6.23. (-)- $N$-Benzoyl 4-chlorophenyl methyl sulfimide (4ae)
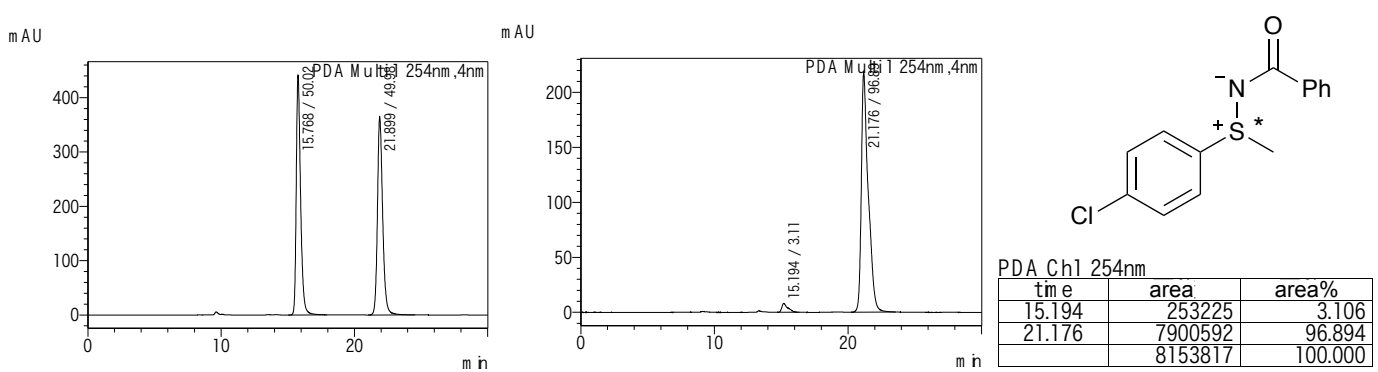

6.24. (-)-N-Benzoyl 4-fluorophenyl methyl sulfimide (4af)
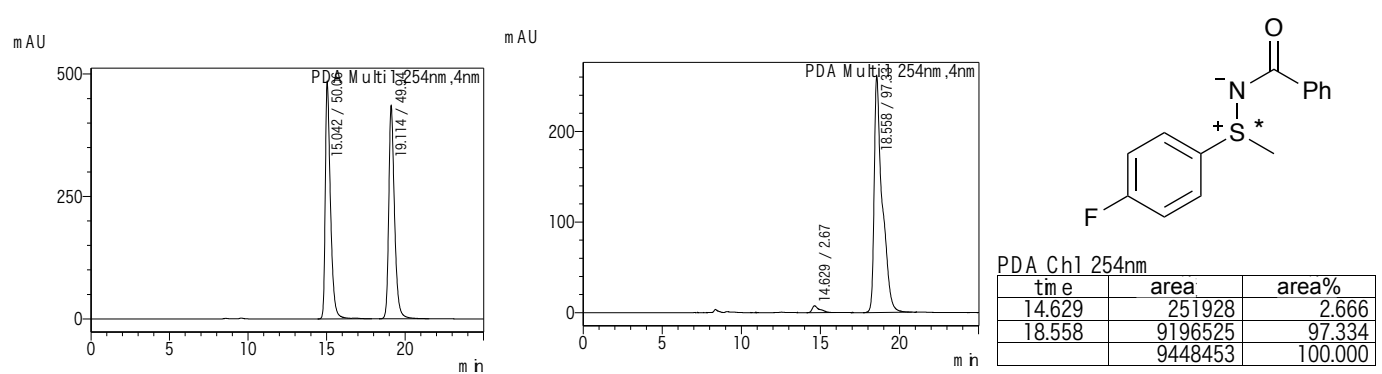
6.25. (-)- $N$-Benzoyl methyl 4-nitrophenyl sulfimide (4ag)
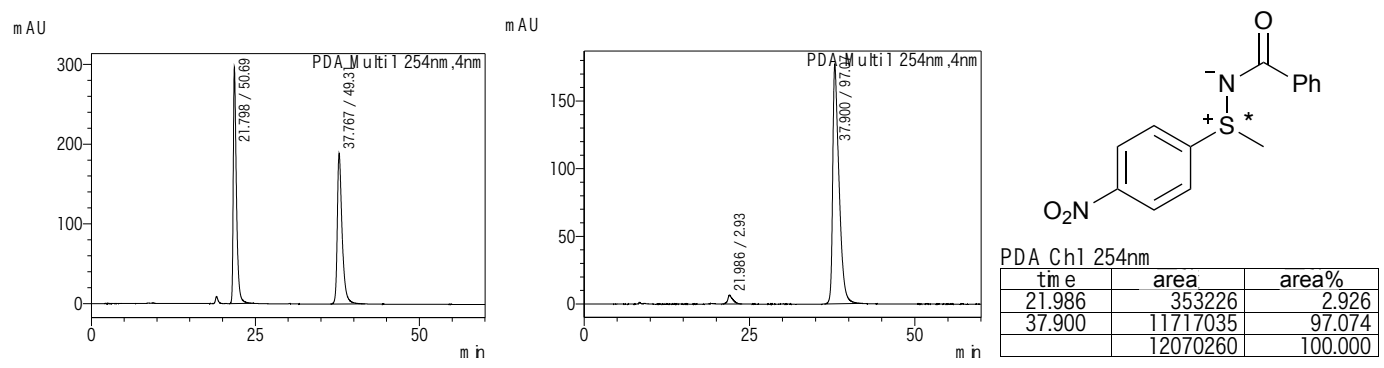

6.26. (-)- $N$-Benzoyl 3-bromophenyl methyl sulfimide (4ah)
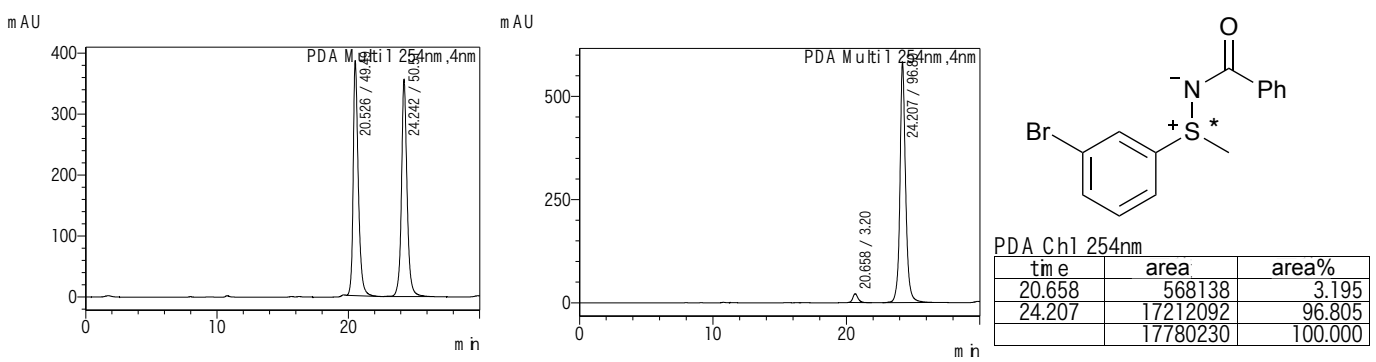

6.27. (-)- $N$-Benzoyl 2-bromophenyl methyl sulfimide (4ai)
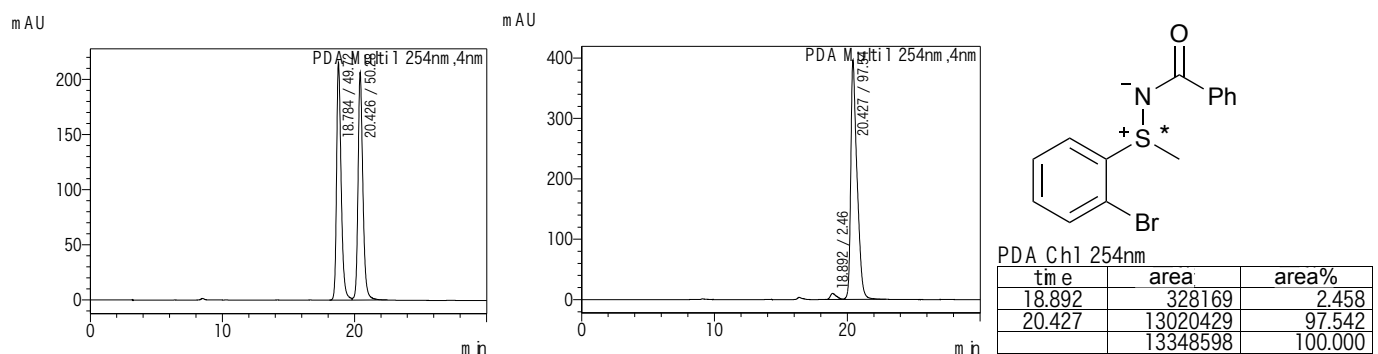

6.28. (-)- $N$-Benzoyl ethyl phenyl sulfimide (4aj)
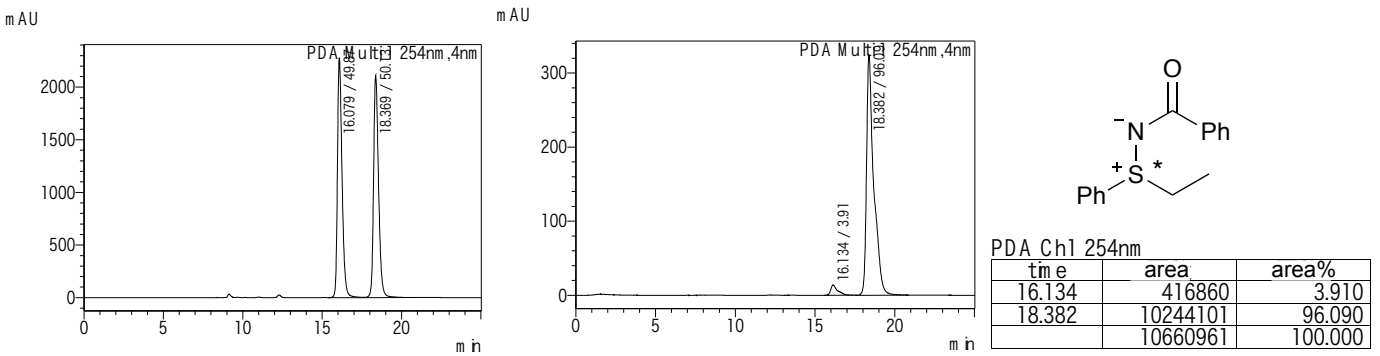
6.29. (-)- $N$-Benzoyl benzyl methyl sulfimide (4al)
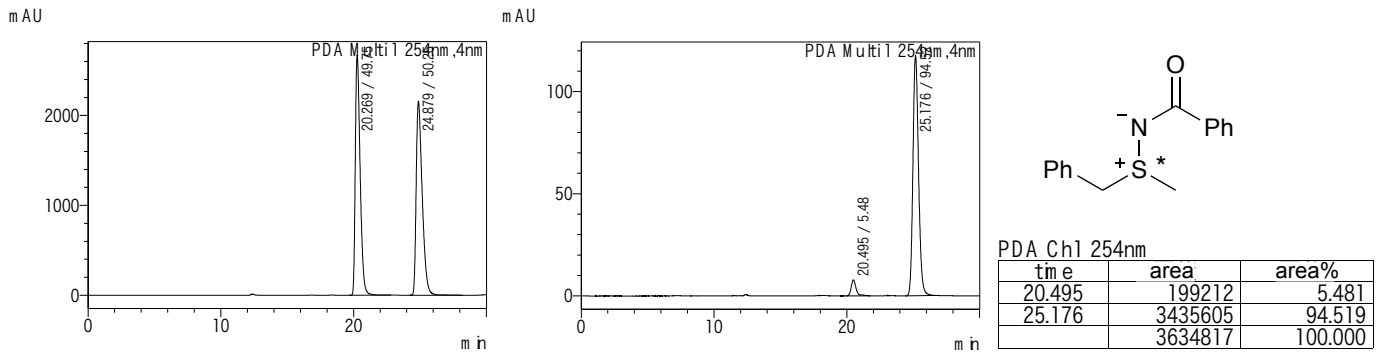

6.30. (+)- $N$-Benzoyl methyl octyl sulfimide (4am)
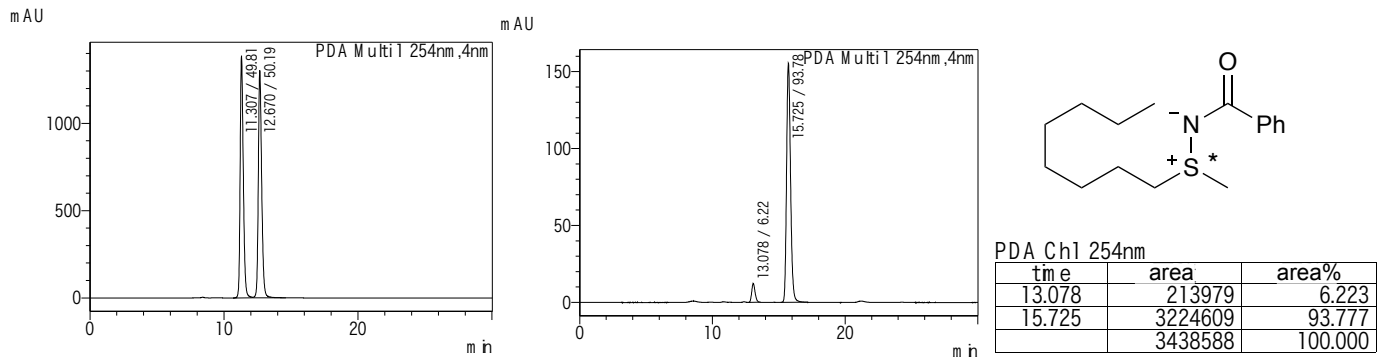

\subsection{1. (+)- $N$-Benzoyl methyl 2-propyl sulfimide (4an)}
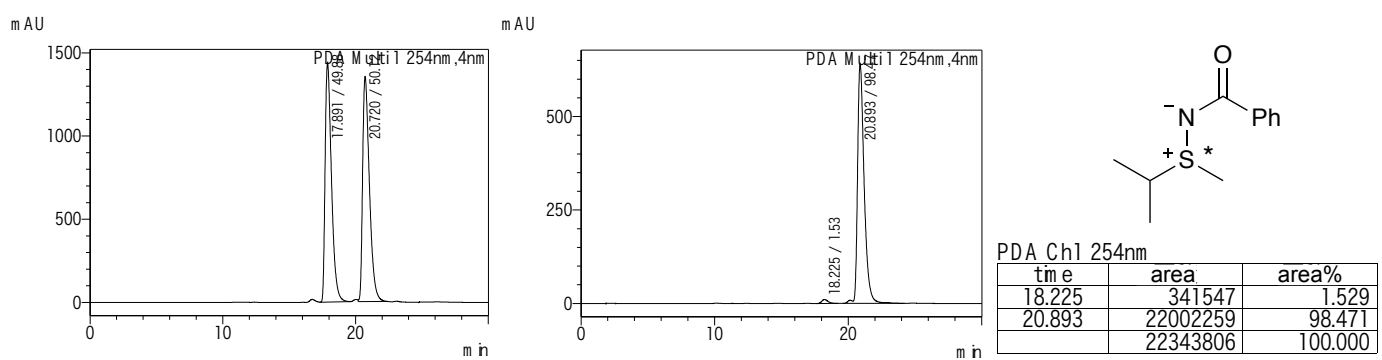

6.32. (+)- $N$-Benzoyl tert-butyl methyl sulfimide (4ao)
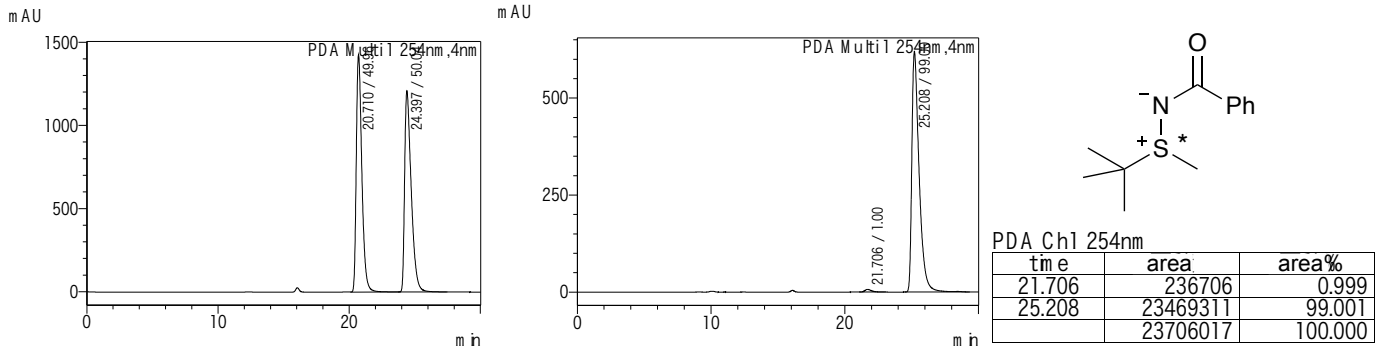


\subsection{3. (-)- $N$-Benzoyl ethyl propyl sulfimide (4ap)}
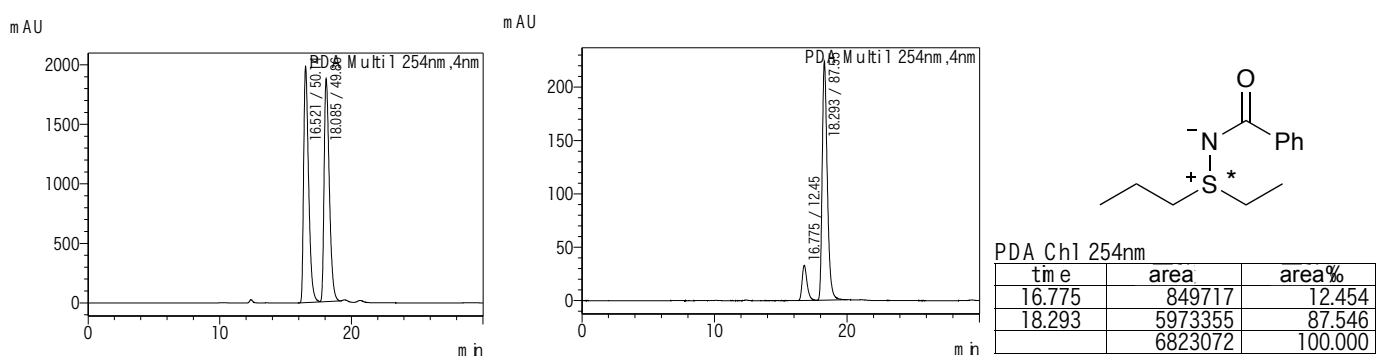

6.34. (+)- $N$-Benzoyl methyl phenyl sulfoximine (5aa)
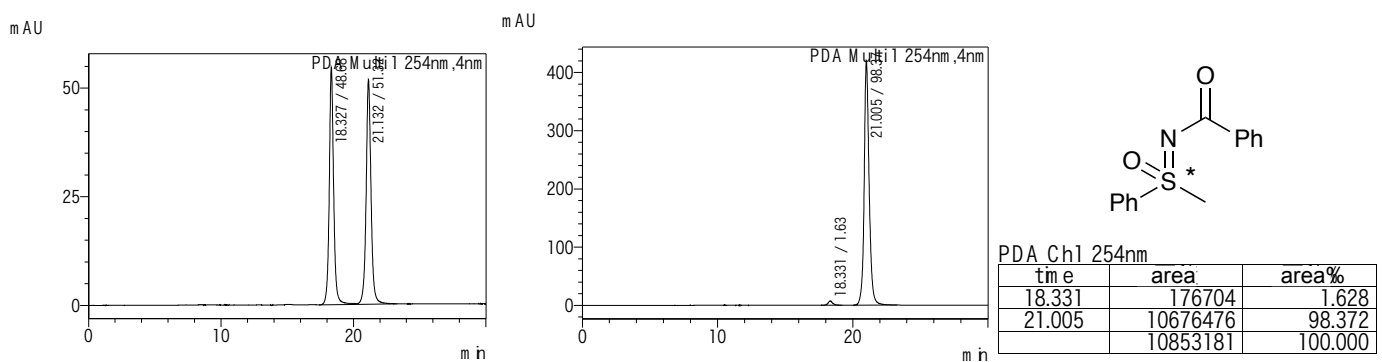

6.35. (R)-(-)- $N$-Acyl methyl phenyl sulfoximine (5pa)
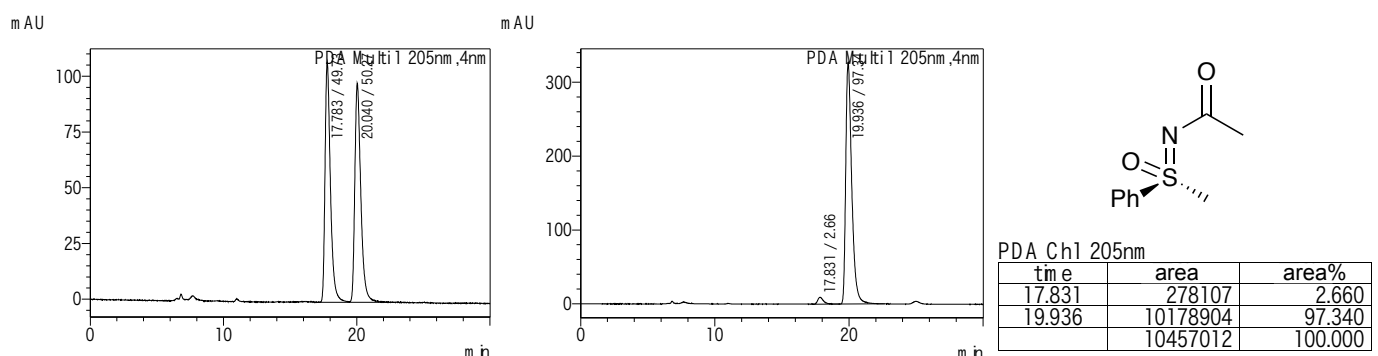


\section{References.}

1. V. Bizet, L. Buglioni, C. Bolm, Angew. Chem. Int. Ed., 2014, 53, 5639.

2. a) Y. Nishioka, T. Uchida, T. Katsuki, Angew. Chem. Int. Ed., 2013, 52, 1739. H. Kawabata, K. Omura, T. Uchida, T. Uchida, Chem. Asian J., 2007, 2, 248; (d) C. Kim, T. Uchida, T. Katsuki, Chem. Commun., 2012, 48, 7188.

3. C. M. M. Hendriks, P. Lamers, J. Engel, C. Bolm, Adv. Synth. Catal. 2013, 355, 3363.

4. C. Bolm, C. P. Hackenberger, O. Simić, M. Verrucci, D. Müller, F. Bienewald, Synthesis, 2002,879 . 